ICH eCTD Specification V 2.0 February 12, 2002

\title{
INTERNATIONAL CONFERENCE ON HARMONISATION OF TECHNICAL REQUIREMENTS FOR REGISTRATION OF PHARMACEUTICALS FOR HUMAN USE
}

\section{ICH M2 EWG}

\section{Electronic Common Technical Document Specification}

This specification has been developed by the ICH M2 Expert Working Group in accordance with the ICH Process as pertains to the M2 EWG. 
ICH eCTD Specification V 2.0 February 12, 2002

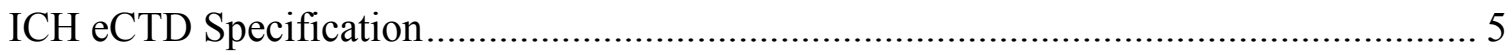

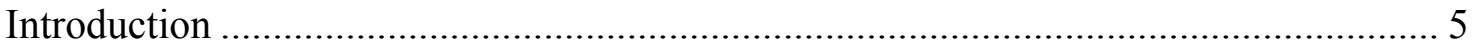

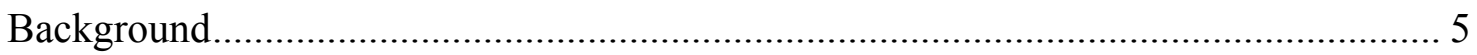

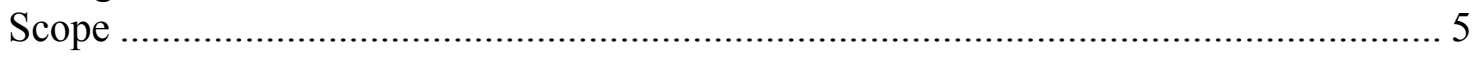

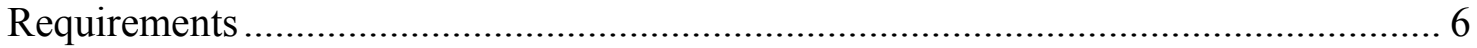

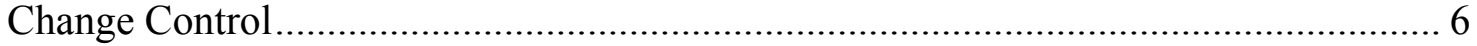

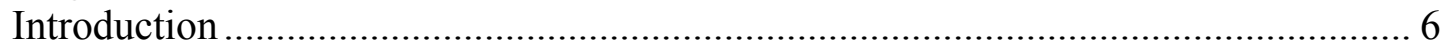

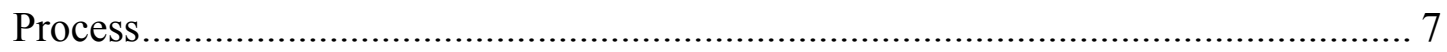

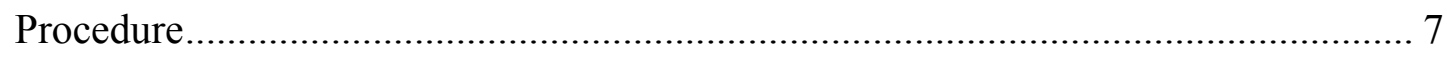

Approach to Documentation and Use of the eCTD Specification............................... 8

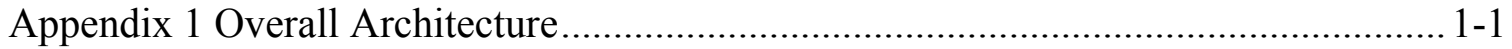

Guiding Design Principles..................................................................................... 1-1

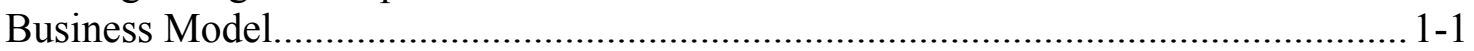

Modular Structure of the eCTD ........................................................................... 1-1

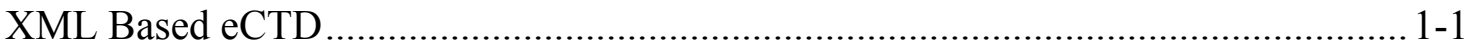

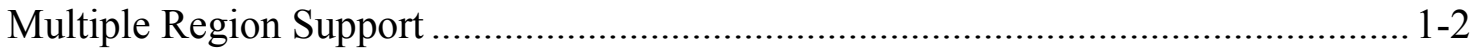

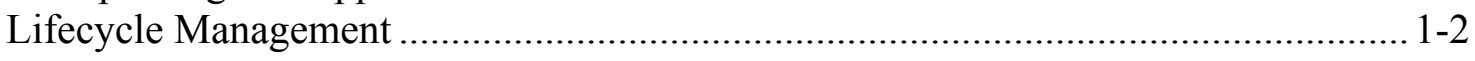

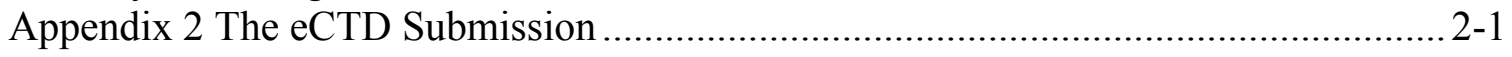

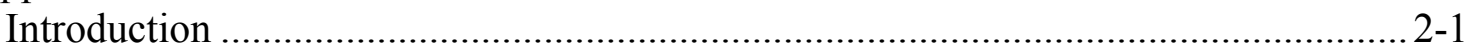

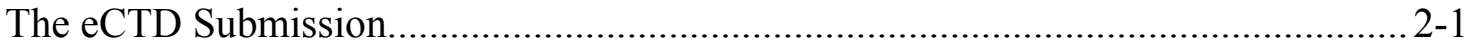

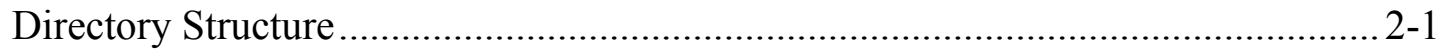

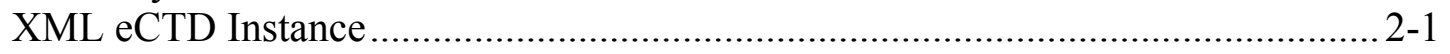

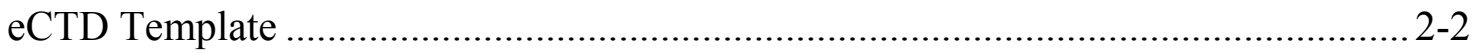

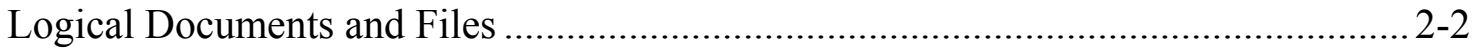

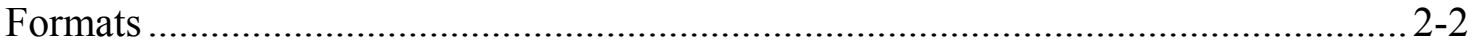

Common Formats ...................................................................................... 2-2

Regional Use of Other Formats ...................................................................... 2-2

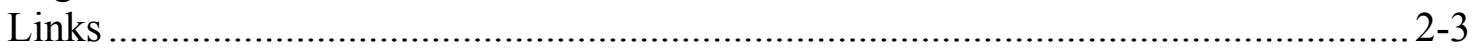

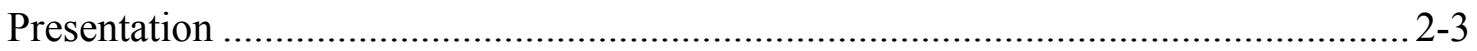

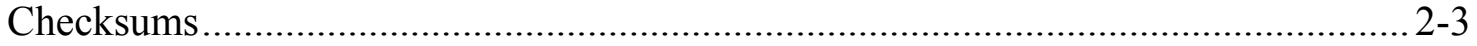

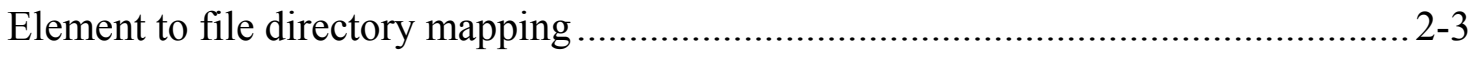

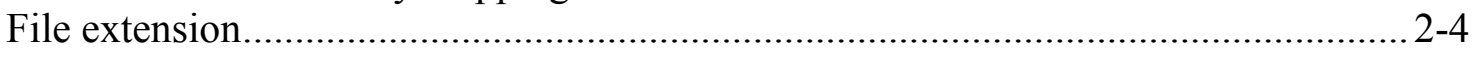

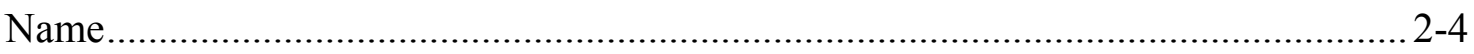

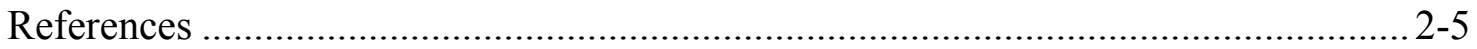

Appendix 3 General Considerations for the CTD Modules......................................... 3-1

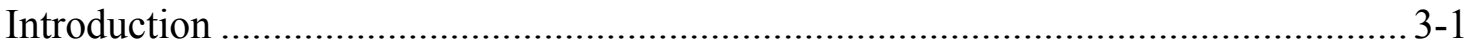

Folder and File Naming Conventions.................................................................. 3-1

Screenshots and folder hierarchy............................................................... 3-2

Module 1 Administrative Information and Prescribing Information.........................3-3

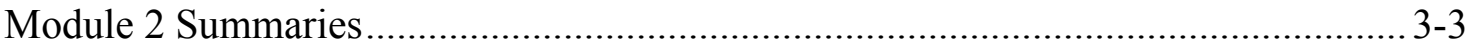

Module 3 Quality..................................................................................... 3-3

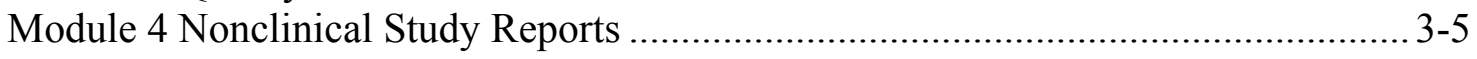

Module 5 Clinical Study Reports ......................................................................... 3-9

Appendix 4 File Organization for the eCTD ........................................................ 4-1

Appendix 5 Region Specific Information Including Transmission and Receipt ............ 5-1 
ICH eCTD Specification V 2.0 February 12, 2002

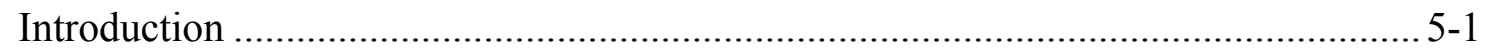

Region specific information: Module 1 ............................................................... 5-1

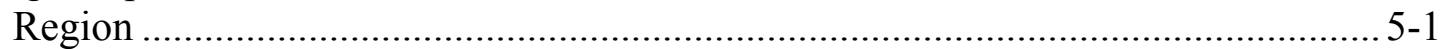

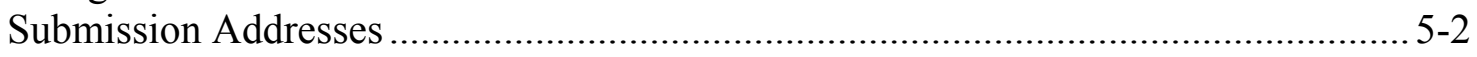

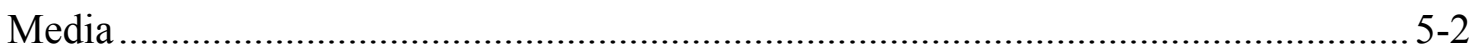

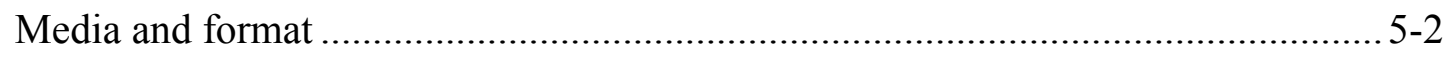

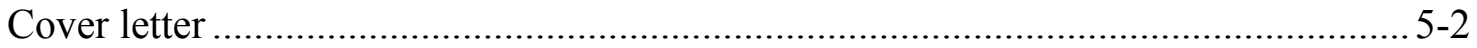

Preparing the media ........................................................................................ 5-3

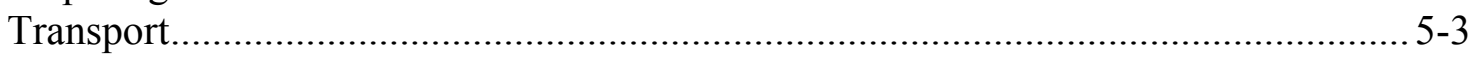

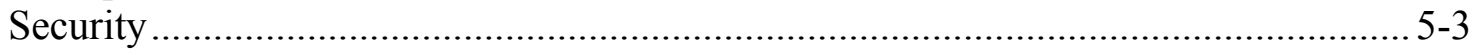

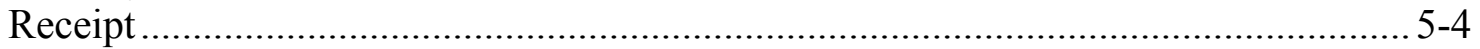

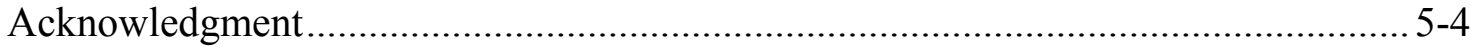

Appendix 6 The eCTD XML Submission ........................................................... 6-1

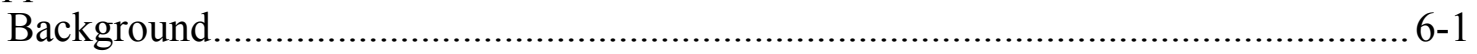

File Names and Directory Structure .............................................................. 6-1

Lifecycle Management ......................................................................................... 6-3

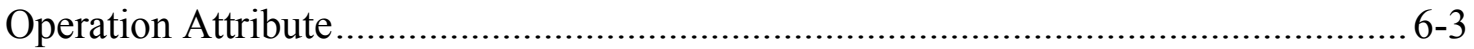

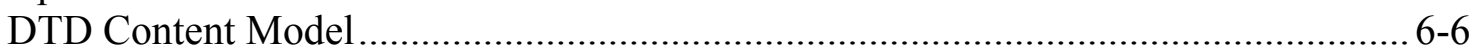

eCTD Element/Attribute Instructions.............................................................. 6-9

Instructions for a Simple New Submission ..................................................... 6-11

Instructions for an Amendment, Supplement or Variation.................................... 6-12

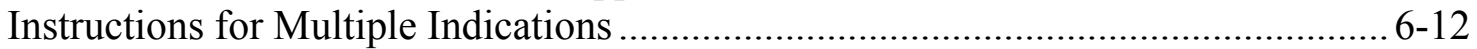

Instructions for Multiple Drug Substances, Manufacturers and Products................. 6-13

Instructions for extending XML eCTD DTD elements..................................... 6-14

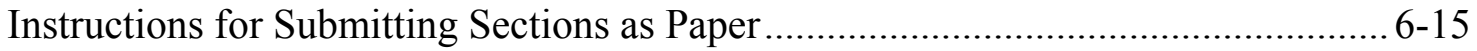

Appendix 7 Specification for Submission Formats ................................................. $7-1$

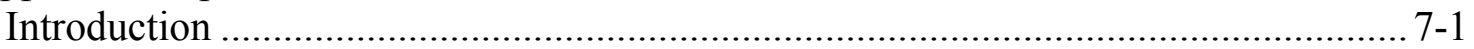

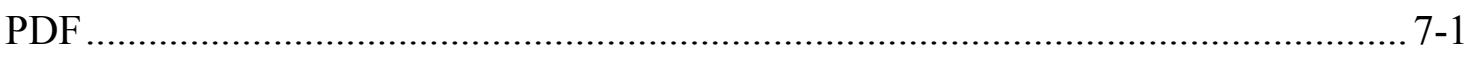

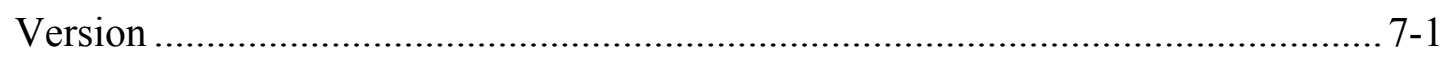

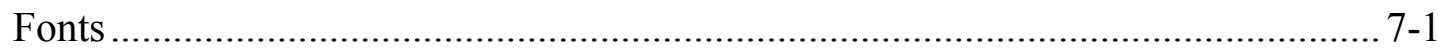

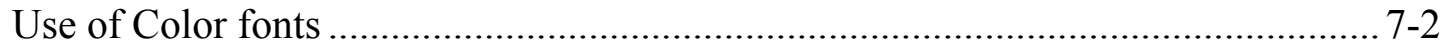

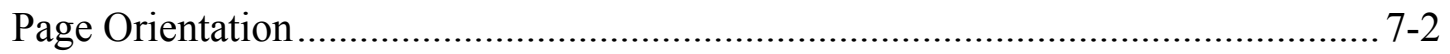

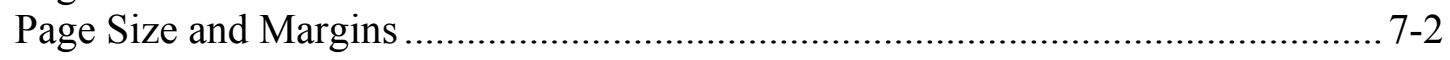

Source of Electronic Document ................................................................. 7-3

Methods for Creating PDF Documents and Images................................................ 7-3

Hypertext Linking and Bookmarks ................................................................... $7-4$

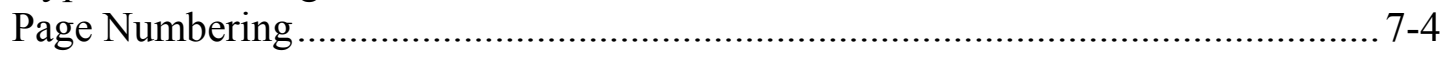

Document Information Fields ................................................................... $7-5$

Open Dialog Box......................................................................................... $7-5$

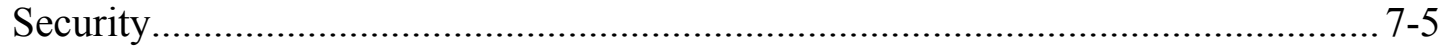

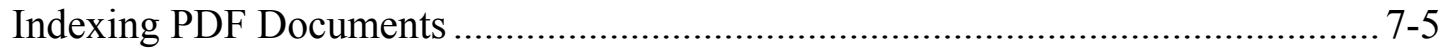

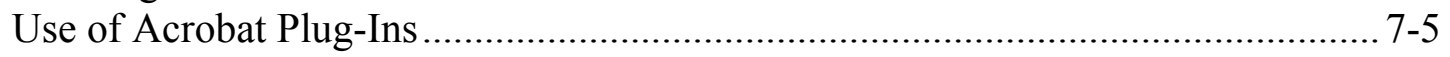

XML Files................................................................................................ $7-6$

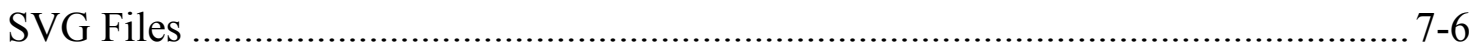

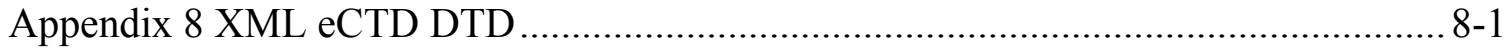

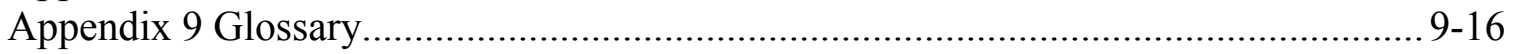


ICH eCTD Specification V 2.0 February 12, 2002

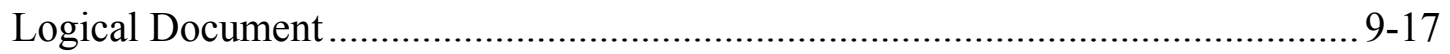

Page 4 
ICH eCTD Specification V 2.0 February 12, 2002

\section{ICH eCTD Specification}

\section{Introduction}

The ICH M4 Expert Working Group (EWG) has defined the Common Technical Document (CTD). The ICH M2 EWG has defined, in the current document, the specification for the Electronic Common Technical Document (eCTD). The eCTD is defined as an interface for industry to Agency transfer of regulatory information while at the same time taking into consideration the facilitation of the creation, review, lifecycle management and archival of the electronic submission. The eCTD specification lists the criteria that will make an electronic submission technically valid. The focus of the specification is to provide the ability to transfer the registration application electronically from industry to a regulatory authority. Industry to industry and Agency to Agency transfer is not addressed.

The specification is divided into a series of main sections followed by a number of appendices in which detailed technical specifications are given. It will provide a mechanism whereby parts of the specification will be updated or adjusted to agreed new technologies or standards on an independent basis without the necessity of updating it all. This aspect will be covered in the chapter Change Control.

\section{Background}

The specification for the eCTD is based upon content defined within the CTD issued by the ICH M4 EWG. The CTD describes the organization of modules, sections and documents. The structure and level of detail specified in the CTD has been used as the basis for defining the eCTD structure and content but where appropriate, additional details have been developed within the eCTD specification.

The philosophy of the eCTD is to utilize open standards. Open standards, including proprietary standards, which through their widespread usage can be considered de facto standards, are deemed to be appropriate in general.

\section{Scope}

The CTD as defined by the M4 EWG does not cover the full submission that is to be made in a region. It describes only modules 2 to 5 , which are common across all regions. It does not describe the content of module 1 the Regional Administrative Information and Prescribing Information nor does it describe documents that can be submitted as amendments or variations to the initial application.

The value of production of a specification for the creation of an electronic submission based only upon the modules described in the CTD would be limited. Therefore, the M2 EWG has produced a specification for the eCTD that is applicable to all modules of initial registration applications and for other submissions of information throughout the lifecycle of the product, such as variations and amendments.

This document describes the parts of the registration application that are common to all regions and some of the lifecycle requirements for products. The parts of the registration 
application that are specific to a region will be covered by regional guidance. However, the backbone has been developed to handle both the regional and common parts of submissions.

\section{Requirements}

The specification is designed to support high-level functional requirements such as the following:

- Copy and paste

- Viewing and printing of documents

- Annotation of documentation

- Facilitate the exporting of information to databases

- Searching within and across applications

- Navigation throughout the eCTD and its subsequent amendments/variations

\section{Change Control}

\section{Introduction}

The specification for the eCTD is likely to change with time. Factors that could affect the content of the specification include, but are not limited to:

- Change in the content of the CTD, either through the amendment of information, at the same level of detail, or by provision of more detailed definition of content and structure

- Change to the regional requirements for applications that are outside the scope of the CTD

- Updating of standards that are already in use within the eCTD

- Identification of new standards that provide additional value for the creation and/or usage of the eCTD

- Identification of new functional requirements

- Experience of use of the eCTD by all parties

The first specification for an eCTD is an ICH M2 Step 4 document. The Specification includes an appendix for the modules of the CTD. Each appendix consists of (or includes) detailed information for the structure and format to be used in preparing a CTD module.

It is understood that technology will continue to evolve at a rapid pace. There could also be changes to the CTD. Information technology capabilities and requirements will also evolve in the pharmaceutical industry and in the regulatory authorities. The change control process described in this section allows the eCTD Specification to be updated to meet new requirements and to take advantage of technology improvements. Each appendix should be updated as needed, independent of the remainder of the document. 


\section{Process}

The eCTD Specification Change Control Board (CCB) is authorized by the ICH Steering Committee to make changes to the eCTD Specification to keep pace with advancing technology. Since the issuance of guidelines is the responsibility of the regulatory authorities, in line with the standards ICH process, the regulatory authorities are the voting members of the CCB. Industry representatives from each of the three regions, and Health Canada as observer, are non-voting members of the CCB. The position of chair of the $\mathrm{CCB}$ rotates on an annual basis among the regulatory authority members.

The three regulatory authorities represented in the ICH M2 Expert Working Group are responsible for initiating changes to the eCTD Specification, based on industry or regulatory input. A change can be proposed by any of the regulatory authorities. A group or individual, not a member of an ICH regulatory authority, can propose a change to the specification, including recommendation for experts to be invited, by submitting the proposal to one of the regional regulatory authorities.

The CCB meets on a regular schedule to discuss, evaluate and agree on proposed changes to the specification. During these meetings the members of the $\mathrm{CCB}$ and other invited parties evaluate the proposed changes. The decision to accept a change to the eCTD Specification is made by a unanimous vote of the regulatory authority representatives.

The agreed changes to the specification will be published for public comment in each region. Comments are collected and considered by the CCB and will be adopted in modified or unmodified form or rejected. The updated part of the eCTD Specification will be agreed upon and signed by the three regional regulatory authorities, and will be published as required in each region. The planned implementation date and transition period for each change in each region is included in the published description of the change. Adopted changes will normally be published on an annual basis except for emergency changes, e.g. an error in critical meta-data, as defined by the CCB which will be published immediately upon adoption. The CCB will provide guidance that will indicate how existing submissions and those currently undergoing late stage-compilation should be updated.

Regulatory authorities will support submissions described by at least two consecutive versions of the eCTD Specification. The regulatory authority intends to keep all versions of the specification as long as needed to process eCTD submissions that are on file with a regulatory authority.

The CCB will establish its meeting schedule at the first meeting of the CCB. The first meeting will be at the same time as the ICH Steering Committee.

\section{Procedure}

Change requests should be submitted to a regulatory authority. Change requests received at least 30 days before a scheduled CCB meeting will be placed on the agenda for that 
meeting. Change requests received less than 30 days before a CCB meeting will be placed on the agenda for the following meeting.

Change requests should contain as much of the following information as possible:

- A description of the problem that the change is intended to solve.

- The proposed solution(s) - this consists of a description of the solution(s) and the text of the changes to affected documents.

- A detailed description of any testing or research that was done to support the solution(s) being proposed.

- Advice on backward compatibility issues, if any.

The CCB will maintain a public list of requests and the status of each request. New change requests will be posted to the list within 30 days of their receipt.

\section{Approach to Documentation and Use of the eCTD Specification}

The approach to the management of the specification for the eCTD is to divide the documentation into a series of independent but related appendices. This will facilitate the maintenance of the specification, as it will not require that all documentation be updated even for a small change to one part of the specification. Each appendix can be updated independently as and when required, thus being able to more readily support the currency of the specification as a whole. 


\section{Appendix 1 Overall Architecture}

\section{Guiding Design Principles}

This appendix defines the basic principles that drove the design and architecture of the eCTD. Detailed specifications are defined in appendices 2 and 6.

\section{Business Model}

The business process to be supported can be described as follow:

$$
\text { Industry <-----> Message <------> Agency }
$$

The business process defines specific requirements for the message.

The primary focus of the eCTD is to provide a data interchange message between the industry and agencies. The industry initiates the process by creating the initial submission in terms of an electronic CTD. Throughout the lifecycle of this process, additional information will be submitted to update or modify the information contained in the initial submission e.g. supplement, amendment, variation etc. The agency can submit acknowledgements, queries and requests to the industry. These are considered simple messages utilizing electronic mail or other transport formats. The overall architecture of the $\mathrm{CTD}$ is designed to provide a commonly agreed upon submission and submission structure that imposes minimal restriction to the industry and agencies.

\section{Modular Structure of the eCTD}

The structure of the electronic submission in terms of organization and navigation should be consistent with the modular structure of the Common Technical Document. The goal of this design principle is to standardize the electronic format of the common parts of the eCTD.

\section{XML Based eCTD}

The XML eCTD DTD (Document Type Definition) defines the overall structure of the submission. The purpose of the XML backbone is two-fold: (1) to manage meta-data for the entire submission and each document within the submission and (2) to constitute a comprehensive table of contents and provide corresponding navigation aids. Meta-data on submission level includes information about submitting and receiving organization, manufacturer, publisher, ID and kind of the submission, and related data items. Examples for meta-data on document level are versioning information, language, descriptive information such as document names, checksums, etc. Details are defined in appendix 6 .

The XML instance of any submission should be created and validated according to the XML eCTD DTD as defined in appendix 8.

The XML eCTD DTD describes the hierarchical structure according to the CTD as defined by the ICH M4 expert working group. It includes multiple hierarchical levels depending on the specific module as defined in the CTD. The actual submission can 
include more hierarchical levels below those defined in the CTD. The XML eCTD instance covers the entire submission including all hierarchical levels and includes references to each individual file.

The submission should include a style sheet that supports presentation of the XML instance, navigation according to the table of contents and provides access to all documents within the submission. A standard style sheet is defined and provided by the ICH M2 EWG. Presentation and navigation via other style sheets on the receiving side should be possible.

The XML eCTD DTD includes a reference for each document to the physical file within the folder structure. The XML eCTD DTD includes attributes for descriptive names of folders and documents.

\section{Multiple Region Support}

The scope of each submission is global according to the Common Technical Document, meaning that modules 2 through 5 of a submission are intended for all regions with the exception of selected documents (e.g. in the quality module), which have a regional scope. Module 1 of a submission is regional in nature.

The DTD as defined by the ICH M2 expert working group specifies the structure of the common parts of the eCTD primarily focusing in module 2 through 5. It allows linking to regional DTDs for module 1 which will be defined by the authorities in each region.

\section{Lifecycle Management}

The applicant creates a submission that is stored in a local repository. The applicant submits the initial submission to the agency, which imports the submission into another local repository. The nature and kind of the local repositories is not within the scope of the eCTD. The initial submission should be self-contained meaning that it includes all documents and no references to other submissions. Regional guidance should be consulted if references to other submissions are needed.

Following the initial submission, the applicant can submit incremental updates such as amendments and variations. Updates can refer to documents in the previous submissions. Updates should be designed in a way that they can be loaded into the repository by fully preserving the initial or previous submission via version control. The XML backbone should include meta-data identifying the update and providing navigation aids to filter for different submission types.

It is preferred that when a Common Technical Document is submitted electronically, the entire submission should be in electronic form with the exception of certain regional forms that currently require written signatures. See appendix 5 for regional requirements. See appendix 6 for a description of how to submit a CTD containing both paper and electronic components. 


\section{Appendix 2 The eCTD Submission}

\section{Introduction}

This appendix specifies the Information Technology aspect of the eCTD Submission. Informally, the eCTD Submission is a directory structure with files including the XML eCTD instance, reports, data and other submission information. The eCTD Submission supports multilingual and multi-region aspects.

\section{The eCTD Submission}

An eCTD Submission is a collection of data objects that follows the eCTD Specification. The main function of the eCTD Submission is data exchange. Information systems would have to be created to process the eCTD Submission. The biggest benefits are expected when the eCTD Submission is loaded into an information system that supports the review process. However, one can view an eCTD Submission with a web browser as it is web ready. In the web environment, the eCTD Submission should be usable without processing in at least in the following ways:

- Standalone: Viewable with a web browser.

- Network: Loadable into a web server.

The eCTD Submission is composed of the following:

- Directory Structure

- XML eCTD instance

- Content files

\section{Directory Structure}

The directory structure is a structure of directories and files. There should be a reasonable maximum number of entries (directories and files) per directory. The directory structure should follow the rules below. The files could be in several formats as specified of below.

The name of the files and directories are identifiers. They should be short. The file names are not intended to convey meta-data, though some meaning in the names helps; i.e., no random names.

Names for directories and files are recommended in Appendix 4. Any directory names and file names that are added to the eCTD submission by the applicant should be descriptive and logical.

\section{XML eCTD Instance}

The instance is in the submission sequence number directory (see appendix 6). The submission sequence number directory should contain at least two files and one or more directories. One of the files in the submission sequence directory is the instance and the other is the MD5 checksum of the instance. The instance is the starting file for the processing by an XML processor. 
The intention is to have links from the instance to leaf files in the eCTD submission as opposed to creating a single XML document that contains the entire eCTD submission. The instance should contain mostly linking facilities to the leaf files. The instance also contains meta-data at the leaf level.

\section{eCTD Template}

The ICH web site includes an eCTD Template that is an empty directory structure with a recommended style sheet. It is an illustration of an eCTD Submission and it is ready to be populated with the applicant data. Appendix 4 defines the directories used to create this template.

\section{Logical Documents and Files}

A logical document is comprised of one or more CTD table of contents sections that together contain the minimum amount of information to be exchanged. In general, the XML eCTD DTD maps explicitly to the CTD table of contents, but there are exceptions where the XML eCTD DTD maps to the level of use designated by the appropriate ICH CTD Implementation Working Group (IWG) instead. Ideally, a logical document consists of a single physical file. In the event the physical file exceeds the recommended maximum file size due to graphics, data content, scanned images, or other large format content, additional files may make up the logical document. Furthermore, if the logical document consists of multiple file formats, then more than one physical file would be needed. An example of such a case would be PDF and XML data that together represent the logical document.

\section{Formats}

Formats should be readable at least for as long as it is needed for the regulatory process. This process could be very long; e.g. 50 years. This points to neutral formats: formal standard, industrial standard, vendor independent, text-like, etc. The format should be

adapted to the type of data. Appendix 7 describes the way in which these files should be constructed.

The list of agreed formats will be updated as technology evolves and new requirements arise. XML will be the preferred format for all types of data.

\section{Common Formats}

The common formats that can be included in an eCTD Submission are:

- Narrative: Portable Document Format (PDF)

- Structured: Extensible Markup Language (XML)

- Graphic: Whenever possible, use PDF. When appropriate or when PDF is not possible, use Joint Photographic Experts Group (JPEG), Portable Network Graphics (PNG), Scalable Vector Graphics (SVG) and Graphics Interchange Format (GIF). Special formats for very high resolutions may be appropriate on a case-by-case basis.

\section{Regional Use of Other Formats}

Regulatory authorities and applicants could agree to use other formats regionally; i.e., 
non-common formats or uses of the common formats in a different way from above. The use of other formats is discouraged and the intention is to use as much as possible the common formats. The intention of the use of other formats is for transition.

There are two classes of transitions:

- Legacy Transition: from the past to the present; i.e., old formats to present formats.

- Future Transition: from the present to the future; i.e., from present formats to new formats. The new formats would normally be candidates for common formats.

\section{Links}

Links among objects in the eCTD Submission should be relative. The intention is to make the eCTD submission self-contained. All literature references introduced by the applicant should be included in the submission, for secondary references (references to a reference), absolute links to external objects can be used.

One can always point to a file. The capacity to point to a specific location within a file depends on the linking technology. Different formats allow for the use of different linking technology. See Appendix 7.

\section{Presentation}

Presentation is closely associated with formats. To associate a style sheet with a file usually one has to use a linking technology. The linking between style sheet (that could be in a separate file) and a data file should be relative. In addition, there is the dimension of media. One file could have several style sheets; the one used depends on the media. For example, there could be one presentation for the screen and another for paper.

\section{Checksums}

The eCTD Submission should contain checksums for each individual file including a checksum file for the eCTD XML instance. Initially, the MD5 Message-Digest Algorithm (MD5) should be used for this purpose. Including a checksum for each individual file provides a number of benefits including:

- The integrity of each file can be verified by comparing the checksum submitted with the file and the computed checksum.

- The checksum can be used to verify that the file has not been altered in the historical archive of the regulatory authority. This is especially useful as the files are migrated from one storage medium to another, as in the case of backup to magnetic tape storage.

\section{Element to file directory mapping}

Follow these rules:

- The rules below for the file and directories take precedence.

- Add the corresponding extension to the file.

- If needed, use a reasonable abbreviation. 


\section{File extension}

All files should have one and only one file extension. The file extension should be used to indicate the format of the file. For example:

$\begin{array}{ll}\text { hello.pdf } & \text { PDF } \\ \text { hello.rtf } & \text { RTF }\end{array}$

The mapping between formats and extensions are:

$\underline{\text { IANA nomenclature }}$

text/css

css

text/html

html or htm

text/xml

$\mathrm{xml}$

application/pdf

application/rtf

pdf

rtf

application/vnd.ms-excel xls

image/jpeg

image/png

jpg

image/gif

png

gif

Non IANA nomenclature

DTD $\mathrm{dtd}$

XPT (SAS) xpt

XSL $\quad$ xSl

The eCTD Submission could use formats not registered with the Internet Assigned Numbers Authority (IANA).

The presence of a format in this list does not imply that it would be considered an acceptable format. For formats absent from this list, widely used mapping between the formats and the extensions should be used.

Future direction: if a mechanism (e.g., standard) becomes available that associate the formats with file extension, it should be considered for this specification.

\section{Name}

Name is a token composed of the following characters:

- Letters "a" to "z [U+0061 to U+007A].

- Digits "0" to "9" [U+0030 to U+0039].

- $\quad$ "-" [HYPHEN-MINUS, U+002D].

The notation "U+" refers to the Unicode [UNICODE] notation.

Correct Names (only the name without the extension):

part-b

myfile

Page 2-4 
hello

Incorrect names (only the name without the extension):

part a (' '; SPACE is not allowed)

myfile.xml ('.'; FULL STOP is not allowed)

hello:pdf (':' ; COLON is not allowed)

part_a (', , LOW LINE is not allowed)

Directory name is a name.

File name is one name followed by one name separated by a

'.' (FULL STOP, U+002E).

Correct file names (with the extension):

myfile.pdf

hello.cml

Incorrect file names (with the extension)::

a part.pdf (' '; SPACE is not allowed)

hello (missing extension)

hello:xml (':' ; COLON is not allowed)

The maximum length of a directory name or a file name is 64 characters. Only lower case letters should be used in all file and directory names. The maximum length of a path is 256 characters. For example, "data/module_1/introduction.html" is the path;

"introduction.html" is a File Name.

Document Name is the first Name in the File Name. For example, "docname" in the file name "docname.ext".

\section{Character encoding}

The character encoding (charset) in order of preference is:

- Unicode UTF-8, Unicode 16 bits [ISO-10646].

- ISO-8859-1 (Latin-1) or appropriate ISO-8859-x; e.g., ISO-8859-7 for Greek.

- The appropriate SHIFT_JIS.

- Other character encoding agreed upon regionally by the regulatory authority and applicant.

\section{References}

[CML] Chemical Markup Language

http://www.xml-cml.org

[CSS2] Cascading Style Sheets, level 2

http://www.w3.org/TR/REC-CSS2

Page 2-5 
[ECMAScript] ECMAScript Language Specification, $3^{\text {rd }}$ edition. ECMA- 262 http://www.ecma.ch/ecma1/STAND/ECMA-262.HTM

[EXCEL] Microsoft Excel

http://www.microsoft.com/office/excel/default.htm

[GIF] Graphics Interchange Format

http://tronche.com/computer-graphics/gif/gif89a.html

[HTML] HTML 4.01 Specification

http://www.w3.org/TR/html4

[IANA] Internet Assigned Numbers Authority

http://www.iana.org

[IMT] Internet Media Types

http://www.isi.edu/in-notes/iana/assignments/media-types/media-types

[ISO-10646] Information Technology -- Universal Multiple-Octet Coded Character Set (UCS) -- Part 1: Architecture and Basic Multilingual

Plane, ISO/IEC 10646-1:1993

[ISO-639] Codes for the representation of names of languages

ISO 639:1988.

http://www.iso.ch/cate/d4766.html

http://www.oasis-open.org/cover/iso639a.html.

[JPEG] Joint Photographic Experts Group

http://www.jpeg.org/public/wg1n1807.txt

[MD5] The MD5 Message-Digest Algorithm

http://ietf.org/rfc/rfc1321.txt

[PDF] Portable Document Format

http://partners.adobe.com/asn/developer/technotes.html\#pdfspec

[PNG] PNG (Portable Network Graphics) Specification Version 1.0 http://www.w3.org/TR/REC-png.html

[RTF] Rich Text Format (RTF) Specification, version 1.6

http://msdn.microsoft.com/library/specs/rtfspec.htm

[SVG] Scalable Vector Graphics (SVG) 1.0 Specification (work in progress) http://www.w3.org/TR/1999/WD-SVG-19991203 
[UNICODE] Unicode Consortium

http://www.unicode.org

[XHTML] XHTML 1.0: The Extensible HyperText Markup Language

http://www.w3.org/TR/WD-html-in-xml

[XML] Extensible Markup Language (XML) 1.0 (Second Edition)

http://www.w3.org/TR/REC-xml.html

[XSL] Extensible Stylesheet Language (XSL)

W3C Candidate Recommendation 21 November 2000 (work in progress)

http://www.w3.org/TR/WD-xsl

[XSLT] XSL Transformations

http://www.w3.org/TR/xslt.html 


\section{Appendix 3 General Considerations for the CTD Modules}

\section{Introduction}

Documents that are provided in the different modules should be formatted as defined by the ICH Common Technical Document. There should also be consistency in the way navigation aids are provided. Within each document, bookmarks and hypertext links from the table of contents should be provided to all tables, figures, publications, and appendices.

Hypertext links should be provided throughout the body of these documents to aid efficient navigation to annotations, related sections, publications, appendices, tables, and figures that are not located on the same page. If a list of references is included at the end of a document, there should be hypertext links to the appropriate publication.

Documents should be generated from electronic source documents and not from scanned material, except where access to the source electronic file is not available or where a signature is required.

\section{Folder and File Naming Conventions}

A folder and file organization is presented in this specification. This could be used in most cases, however applicants may modify this specification where appropriate. ${ }^{1}$ For example, to include an additional folder for information where an appropriate folder name is not available in the eCTD specification. It is recommended that applicants maintain folder names listed in this specification. This should not be interpreted to mean that the actual eCTD XML DTD should be changed or altered in any way.

The maximum length of a folder or file name is 64 characters including the extension. Folder or file names should be written in lower case only. All files should have one and only one file extension. The file extension should be used to indicate the format of the file. More details on the naming conventions are given in appendix 2, and examples in appendix 4 .

Typically, the file name would be the applicant's internal numbering or naming convention for the studies. The following table gives an example how files could be named.

\footnotetext{
${ }^{1}$ Regulatory authorities should be notified of additions and changes to the folder structure according to regional guidance.
} 
Table 3-1

\begin{tabular}{|l|l|}
\hline \multicolumn{1}{|c|}{ Description } & \multicolumn{1}{c|}{ File Name } \\
\hline Study Report 1 & study-report-1.pdf \\
\hline Study Report 2 & study-report-2.pdf \\
\hline$\ldots$ & $\ldots$ \\
\hline Study Report n & study-report-n.pdf \\
\hline
\end{tabular}

Data listings can be included as part of a study report document or as a separate appendix. An example of such file names follows.

Table 3-2

\begin{tabular}{|l|l|}
\hline \multicolumn{1}{|c|}{ Description } & \multicolumn{1}{c|}{ File Name } \\
\hline Study Report 1 & study-report-1.pdf \\
\hline Study Report 1 Data & study-report-1-data.pdf \\
\hline Study Report 2 & study-report-2.pdf \\
\hline Study Report 2 Data & study-report-2-data.pdf \\
\hline ... & $\ldots$ \\
\hline Study Report n & study-report-n.pdf \\
\hline Study Report n Data & study-report-n-data.pdf \\
\hline
\end{tabular}

Regional requirements can provide for the submission of the data listings as a data file. Reference should be made to regional guidances.

\section{Screenshots and folder hierarchy}

Screenshots are provided in the following chapters for all modules down to the level of hierarchy as described in this appendix. The representations are in alphabetical order due to the nature of the computer operating system and are therefore not entirely consistent with the sequence of the CTD. In a web browser the content will appear in the order of the CTD table of contents.

Detailed options on the folders and files are provided in appendix 4 in case the applicant chooses to submit more granular documents. It is not mandatory to use the full folder hierarchy. Empty directories can be omitted. 


\section{Module 1 Administrative Information and Prescribing Information}

The name of the folder for module 1 should be module-1.

This module contains administrative information that is unique for each region. Regional guidance will provide the specific instructions on how to provide the administrative forms and detailed prescribing information. Please refer to appendix 5 when preparing module 1 .

\section{Module 2 Summaries}

The files in this module should be provided as PDF text with the exception of a few embedded images, when needed. The name of the folder for module 2 should be module-2. The folders in module 2 should be named as follows.

Table 3-3

\begin{tabular}{|l|l|l|}
\hline $\begin{array}{c}\text { Section in } \\
\text { CTD }\end{array}$ & \multicolumn{1}{|c|}{ Description } & \multicolumn{1}{c|}{ Folder Name } \\
\hline 2.3 & Quality Overall Summary & quality- overall-summary \\
\hline 2.6 & $\begin{array}{l}\text { Nonclinical Written and } \\
\text { Tabulated Summary }\end{array}$ & nonclinical-summary \\
\hline 2.7 & Clinical Summary & clinical-summary \\
\hline
\end{tabular}

Other sections at this level not listed above can typically be submitted as individual files.

The folder hierarchy for module 2 is presented in the screenshot in figure $3-1$.

Figure 3-1 Screenshot of the folder structure of module 2

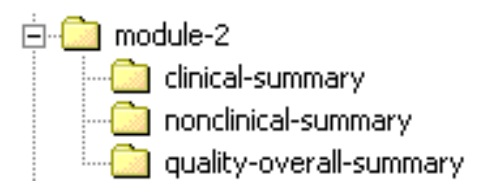

\section{Module 3 Quality}

The name of the folder for module 3 should be module-3. The folders in module 3 should be named as follows.

Table 3-4

\begin{tabular}{|l|l|l|}
\hline $\begin{array}{c}\text { Section in } \\
\text { CTD }\end{array}$ & \multicolumn{1}{|c|}{ Description } & \multicolumn{1}{|c|}{ Folder Name } \\
\hline 3.2 & Body of Data & body-of-data \\
\hline 3.2.S & Drug Substance & drug-substance \\
\hline
\end{tabular}




\begin{tabular}{|c|c|c|}
\hline $\begin{array}{l}\text { Section in } \\
\text { CTD }\end{array}$ & Description & Folder Name \\
\hline $3.2 . \mathrm{S}$ & $\begin{array}{l}\text { Drug Substance [Drug Substance } \\
\text { Name] [Manufacturer] }{ }^{2}\end{array}$ & substance-1-manufacturer-1 \\
\hline 3.2.S.1 & General Information & general-information \\
\hline 3.2.S.2 & Manufacture & manufacture \\
\hline 3.2.S.3 & Characterisation & characterisation \\
\hline 3.2.S.4 & Control of Drug Substance & control-drug-substance \\
\hline 3.2.S.4.1 & Analytical Procedures & analytical-procedures \\
\hline 3.2.S.4.2 & Validation Analytical Procedures & validation-analyt-procedures \\
\hline 3.2.S.7 & Stability & stability \\
\hline 3.2.P & Drug Product $^{3}$ & drug-product \\
\hline 3.2.P & Product 1 & product-1 \\
\hline 3.2.P.3 & Manufacture & manufacture \\
\hline 3.2.P.4 & Control of Excipients & control-excipients \\
\hline 3.2.P.4 & Excipient 1 & excipient-1 \\
\hline 3.2.P.5 & Control of Drug Product & control-drug-product \\
\hline 3.2.P.5.1 & Analytical Procedures & analytical-procedures \\
\hline 3.2.P.5.2 & Validation Analytical Procedures & validation-analyt-procedures \\
\hline 3.2.P.8 & Stability & stability \\
\hline 3.2.A & Appendices & appendices \\
\hline 3.2.A.1 & Facilities and Equipment & facilities-and-equipment \\
\hline 3.2.A.2 & Adventitious Agents Safety Evaluation & adventitious-agents \\
\hline 3.2.A.3 & Novel Excipient $1^{4}$ & novel-excipient-name-1 \\
\hline 3.2.R & Regional Information ${ }^{5}$ & regional-information \\
\hline
\end{tabular}

\footnotetext{
${ }^{2}$ Each drug substance-manufacturer should be placed in a separate subordinate folder. Folders and files should be created for each drug substance-manufacturer section included in the submission in accordance with the hierarchy identified in the following chapters.

${ }^{3}$ Each drug product should be placed in a separate subordinate folder. Folders and files should be created for each drug product section included in the submission in accordance with the hierarchy identified in the following chapters. Reference should be made to regional guidance to determine whether the inclusion of multiple products within a single application is considered appropriate.

${ }^{4}$ The folder name should include the name of the novel excipient, abbreviated as necessary to remain within the 64 character limit.
} 


\begin{tabular}{|l|l|l|}
\hline $\begin{array}{c}\text { Section in } \\
\text { CTD }\end{array}$ & \multicolumn{1}{|c|}{ Description } & \multicolumn{1}{c|}{ Folder Name } \\
\hline 3.3 & Literature References & references \\
\hline
\end{tabular}

The folder hierarchy for module 3 is presented in the screenshot in figure 3-2.

\section{Figure 3-2 Screenshot of the folder structure of module 3}

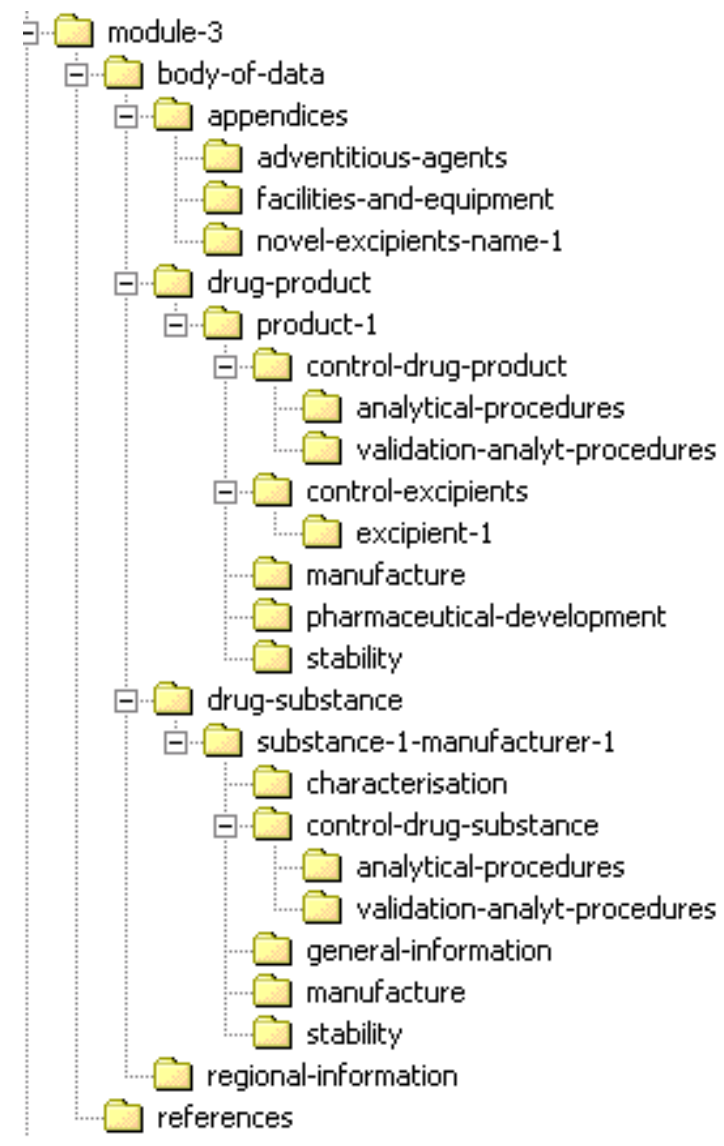

\section{Module 4 Nonclinical Study Reports}

The name of the folder for module 4 should be module- 4 . The folders in module 4 should be named as follows.

Table 3-5

\begin{tabular}{|l|l|l|}
\hline $\begin{array}{c}\text { Section } \\
\text { in CTD }\end{array}$ & Description & Folder Name \\
\hline
\end{tabular}

\footnotetext{
${ }^{5}$ This folder should be included where regional information is appropriate. Reference should be made to regional guidance for the types of information to be included in this section.
} 


\begin{tabular}{|c|c|c|}
\hline $\begin{array}{l}\text { Section } \\
\text { in CTD }\end{array}$ & Description & Folder Name \\
\hline 4.2 & Study Reports & study-reports \\
\hline 4.2 .1 & Pharmacology & pharmacology \\
\hline 4.2 .1 .1 & Primary Pharmacodynamics & Primary-pharmacodynamics \\
\hline 4.2 .1 .2 & Secondary Pharmacodynamics & secondary-pharmacodynamics \\
\hline 4.2 .1 .3 & Safety Pharmacology & safety-pharmacology \\
\hline 4.2.1.4 & Pharmacodynamic Drug Interactions & pd-drug-interactions \\
\hline 4.2 .2 & Pharmacokinetics & pharmacokinetics \\
\hline 4.2.2.1 & $\begin{array}{l}\text { Analytical Methods and Validation } \\
\text { Reports (if separate reports are } \\
\text { available) }\end{array}$ & analyt-methods-validation \\
\hline 4.2 .2 .2 & Absorption & absorption \\
\hline 4.2.2.3 & Distribution & distribution \\
\hline 4.2 .2 .4 & Metabolism & metabolism \\
\hline 4.2 .2 .5 & Excretion & excretion \\
\hline 4.2.2.6 & $\begin{array}{l}\text { Pharmacokinetic Drug Interactions } \\
\text { (nonclinical) }\end{array}$ & pk-drug-interactions \\
\hline 4.2 .2 .7 & Other Pharmacokinetic Studies & other-pk-studies \\
\hline 4.2 .3 & Toxicology & toxicology \\
\hline 4.2.3.1 & $\begin{array}{l}\text { Single-Dose Toxicity (in order by } \\
\text { species, by route) }\end{array}$ & single-dose-toxicity \\
\hline 4.2.3.2 & $\begin{array}{l}\text { Repeat-Dose Toxicity (in order by } \\
\text { species, by route, by duration, including } \\
\text { supportive toxicokinetic evaluations) }\end{array}$ & repeat-dose-toxicity \\
\hline 4.2 .3 .3 & Genotoxicity & genotoxicity \\
\hline 4.2.3.3.1 & In vitro & in-vitro \\
\hline 4.2 .3 .3 .2 & $\begin{array}{l}\text { In vivo (including supportive } \\
\text { toxicokinetics evaluations) }\end{array}$ & in-vivo \\
\hline 4.2.3.4 & $\begin{array}{l}\text { Carcinogenicity (including supportive } \\
\text { toxicokinetics evaluations) }\end{array}$ & carcinogenicity \\
\hline 4.2.3.4.1 & $\begin{array}{l}\text { Long-term studies (in order by species, } \\
\text { including range-finding studies that } \\
\text { cannot be appropriately include under }\end{array}$ & long-term-studies \\
\hline
\end{tabular}




\begin{tabular}{|c|c|c|}
\hline $\begin{array}{l}\text { Section } \\
\text { in CTD }\end{array}$ & Description & Folder Name \\
\hline & $\begin{array}{l}\text { repeat-dose toxicity or } \\
\text { pharmacokinetics) }\end{array}$ & \\
\hline 4.2.3.4.2 & $\begin{array}{l}\text { Short-or medium-term studies } \\
\text { (including range-finding studies that } \\
\text { cannot be appropriately include under } \\
\text { repeat-dose toxicity or } \\
\text { pharmacokinetics) }\end{array}$ & short-medium-term-studies \\
\hline 4.2 .3 .4 .3 & Other studies & other-studies \\
\hline 4.2.3.5 & $\begin{array}{l}\text { Reproductive and Developmental } \\
\text { Toxicity (including range-finding } \\
\text { studies and supportive toxicokinetics } \\
\text { evaluations) }\end{array}$ & repro-development-toxicity \\
\hline 4.2.3.5.1 & $\begin{array}{l}\text { Fertility and early embryonic } \\
\text { development }\end{array}$ & fertility-embryonic-develop \\
\hline 4.2 .3 .5 .2 & Embryo-fetal development & embryo-fetal-develop \\
\hline 4.2.3.5.3 & $\begin{array}{l}\text { Prenatal and postnatal development, } \\
\text { including maternal function }\end{array}$ & pre-postnatal-develop \\
\hline 4.2.3.5.4 & $\begin{array}{l}\text { Studies in which the offspring (juvenile } \\
\text { animals) are dosed and/or further } \\
\text { evaluated }\end{array}$ & juvenile \\
\hline 4.2 .4 & Local Tolerance & local-tolerance \\
\hline 4.2 .5 & Other Toxicity Studies (if available) & other-toxicity-studies \\
\hline 4.2.5.1 & Antigenicity & antigenicity \\
\hline 4.2.5.2 & Immunotoxicity & immunotoxicity \\
\hline 4.2 .5 .3 & $\begin{array}{l}\text { Mechanistic studies (if not included } \\
\text { elsewhere) }\end{array}$ & mechanistic-studies \\
\hline 4.2 .5 .4 & Dependence & dependence \\
\hline 4.2 .5 .5 & Metabolites & metabolites \\
\hline 4.2.5.6 & Impurities & impurities \\
\hline 4.2 .5 .7 & Other & other \\
\hline 4.3 & Literature References & references \\
\hline
\end{tabular}


The folder hierarchy for module 4 is presented in the screenshot in figure 3-3.

Page 3-8 


\section{Figure 3-3 Screenshot of the folder structure of module 4}

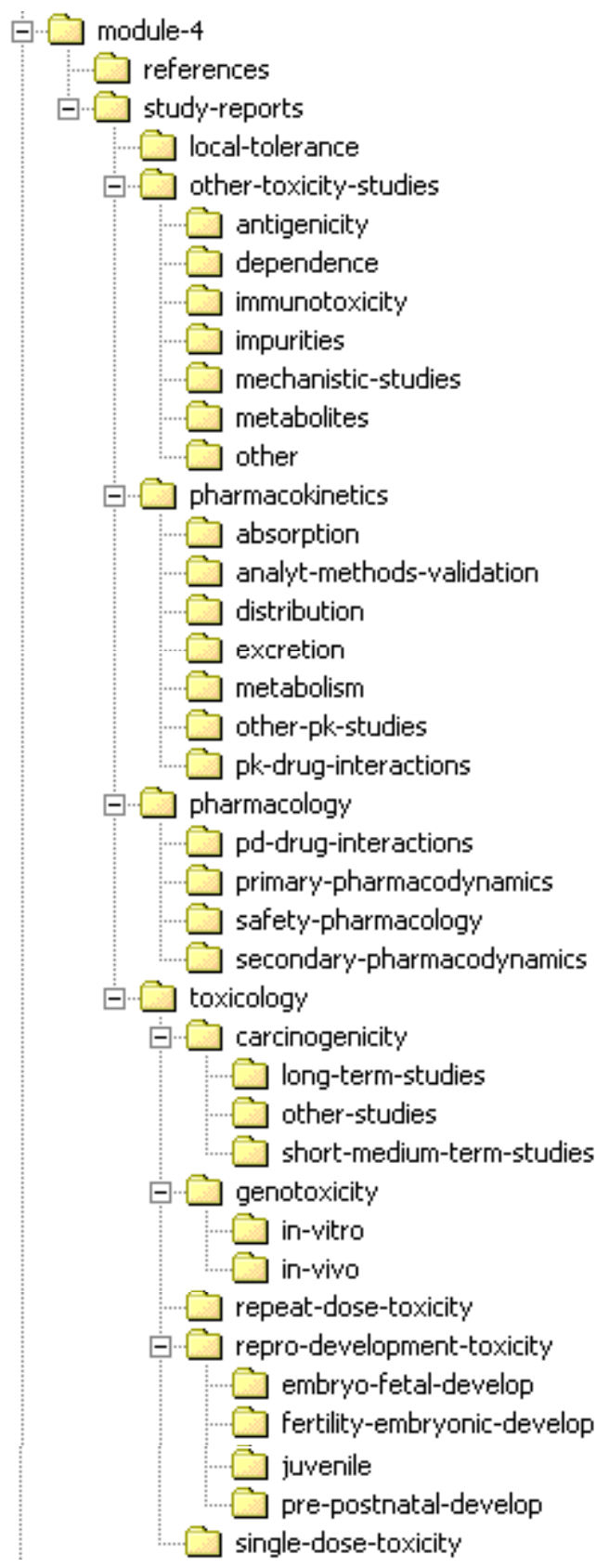

\section{Module 5 Clinical Study Reports}

The name of the folder for module 5 should be module-5. The folders in module 5 should be named as follows. 
Table 3-6

\begin{tabular}{|c|c|c|}
\hline $\begin{array}{l}\text { Section in } \\
\text { CTD }\end{array}$ & Description & Folder Name \\
\hline 5.3 & Clinical Study Reports & clinical-study-reports \\
\hline 5.3 .1 & Reports of Biopharmaceutic Studies & biopharmaceutic-studies \\
\hline 5.3 .1 .1 & Bioavailaility (BA) Study Reports & bioavailability \\
\hline 5.3.1.2 & $\begin{array}{l}\text { Comparative BA and Bioequivalence } \\
\text { (BE) Study Reports }\end{array}$ & comparative-ba-be \\
\hline 5.3.1.3 & $\begin{array}{l}\text { In vitro - In vivo Correlation Study } \\
\text { Reports }\end{array}$ & in-vitro-in-vivo \\
\hline 5.3.1.4 & $\begin{array}{l}\text { Reports of Bioanalytical and Analytical } \\
\text { Methods for Human Studies }\end{array}$ & bioanalyt-analyt-methods \\
\hline 5.3 .2 & $\begin{array}{l}\text { Reports of Studies Pertinent to } \\
\text { Pharmacokinetics using Human } \\
\text { Biomaterials }\end{array}$ & pk-human-biomaterials \\
\hline 5.3 .2 .1 & Plasma Protein Binding Study Reports & plasma-protein-binding \\
\hline 5.3.2.2 & $\begin{array}{l}\text { Reports of Hepatic Metabolism and } \\
\text { Interaction Studies }\end{array}$ & hepatic-metab-interactions \\
\hline 5.3.2.3 & $\begin{array}{l}\text { Reports of Studies Using Other Human } \\
\text { Biomaterials }\end{array}$ & other-human-biomaterials \\
\hline 5.3 .3 & $\begin{array}{l}\text { Reports of Human Pharmacokinetic } \\
\text { (PK) Studies }\end{array}$ & pk-studies \\
\hline 5.3.3.1 & $\begin{array}{l}\text { Healthy Subject PK and Initial } \\
\text { Tolerability Study Reports }\end{array}$ & healthy-subject-pk \\
\hline 5.3.3.2 & $\begin{array}{l}\text { Patient PK and Initial Tolerability } \\
\text { Study Reports }\end{array}$ & patient-pk \\
\hline 5.3 .3 .3 & Intrinsic Factor PK Study Reports & intrinsic-factor-pk \\
\hline 5.3 .3 .4 & Extrinsic Factor PK Study Reports & extrinsic-factor-pk \\
\hline 5.3 .3 .5 & Population PK Study Reports & population-pk \\
\hline 5.3 .4 & $\begin{array}{l}\text { Reports of Human Pharmacodynamic } \\
\text { (PD) Studies }\end{array}$ & human-pd-studies \\
\hline 5.3.4.1 & $\begin{array}{l}\text { Healthy Subject PD and PK/PD Study } \\
\text { Reports }\end{array}$ & healthy-subject-pd \\
\hline 5.3 .4 .2 & Patient PD and PK/PD Study Reports & patient-pd \\
\hline 5.3 .5 & Reports of Efficacy and Safety Studies & efficacy-safety \\
\hline
\end{tabular}




\begin{tabular}{|l|l|l|}
\hline $\begin{array}{c}\text { Section in } \\
\text { CTD }\end{array}$ & \multicolumn{1}{|c|}{ Description } & \multicolumn{1}{|c|}{ Folder Name } \\
\hline & "Indication 1" & indication-1 \\
\hline & "Indication 2" & indication-2 \\
\hline 5.3 .5 .1 & $\begin{array}{l}\text { "Indication 3" } \\
\text { Study Reports of Controlled Clinical } \\
\text { Indication }\end{array}$ & $\begin{array}{l}\text { indication-3 } \\
\text { controlled-studies }\end{array}$ \\
\hline 5.3 .5 .2 & $\begin{array}{l}\text { Study Reports of Uncontrolled Clinical } \\
\text { Studies }\end{array}$ & uncontrolled-studies \\
\hline 5.3 .5 .3 & $\begin{array}{l}\text { Reports of Analyses of Data from More } \\
\text { than One Study }\end{array}$ & multistudy-analyses \\
\hline 5.3 .5 .4 & Other Study Reports & other-studies \\
\hline 5.3 .6 & Reports of Postmarketing Experience & postmarketing-experience \\
\hline 5.3 .7 & $\begin{array}{l}\text { Case Report Forms and Individual } \\
\text { Patient Listings }\end{array}$ & crfs-patient-listings \\
\hline & "Study 1" & study-1 \\
\hline 5.4 & "Study 2" & study-2 \\
\hline & "Study n" & study-n \\
\hline & references \\
\hline
\end{tabular}

${ }^{6}$ This folder contains as many folders as studies are included. The folders should be named as indicated. The content of the folders should follow regional guidance. 
The folder hierarchy for module 5 is presented in the screenshot in figure 3-4.

\section{Figure 3-4 Screenshot of the folder structure of module 5}

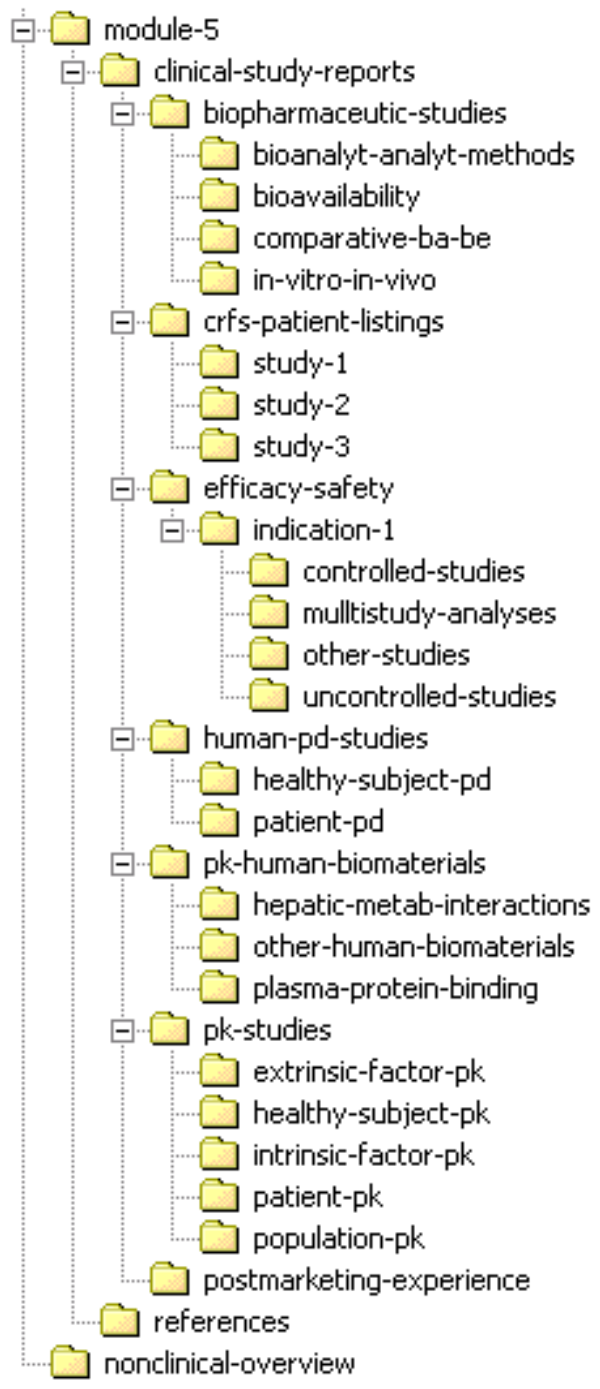




\section{Appendix 4 File Organization for the eCTD}

Each item in the file organization table that is included in this appendix includes the following information: The table below covers files that constitute the backbone itself plus necessary additional files to make the submission complete, readable and processable.

Where file names are presented in italics typically applicants would substitute these with file names in accordance with their own naming conventions.

\begin{tabular}{|l|l|l|}
\hline \multirow{3}{*}{$\begin{array}{l}\text { Sequential } \\
\text { number }\end{array}$} & $\begin{array}{l}\text { Each item in the table has a unique sequentially assigned reference number. These reference } \\
\text { numbers can change with each version of this appendix. }\end{array}$ \\
\cline { 2 - 3 } & Number & CTD section number \\
\cline { 2 - 3 } & Title & CTD title \\
\cline { 2 - 3 } & Element & Element name in the Backbone \\
\cline { 2 - 3 } & File/Directory & $\begin{array}{l}\text { Full path of the File/Directory. The file extension corresponds to the file type; i.e., the "pdf" } \\
\text { extension is only illustrative. Refer to Table 6.1, Appendix 6 for details for the head of the } \\
\text { path name }\end{array}$ \\
\cline { 2 - 3 } & Comment & Comments \\
\hline
\end{tabular}




\begin{tabular}{|l|l|l|}
\hline \multirow{3}{*}{1} & Number & \\
\hline \multirow{3}{*}{1} & Title & \\
\cline { 2 - 3 } & Element & \\
\cline { 2 - 3 } & File & index.xml \\
\hline & Comment & This is Backbone \\
\hline \multirow{3}{*}{2} & Number & \\
\hline \multirow{3}{*}{2} & Title & \\
\cline { 2 - 3 } & Element & \\
\hline & File & index-md5.txt \\
\cline { 2 - 3 } & Comment & The MD5 of the Backbone \\
\hline
\end{tabular}




\begin{tabular}{|c|c|c|}
\hline \multirow{5}{*}{3} & Number & 1 \\
\hline & Title & Administrative Information and Prescribing Information \\
\hline & Element & m1-administrative-information-and-prescribing-information \\
\hline & Directory & module-1 \\
\hline & Comment & Only one of the regional directory is needed \\
\hline \multirow{5}{*}{4} & Number & \\
\hline & Title & \\
\hline & Element & \\
\hline & Directory & module-1/eu \\
\hline & Comment & $\begin{array}{l}\text { EU directory : In addition to the appropriate regional documents, the regional xml instance should be located in this } \\
\text { folder. Refer to regional guidance for details }\end{array}$ \\
\hline \multirow{5}{*}{5} & Number & \\
\hline & Title & \\
\hline & Element & \\
\hline & Directory & module-1/jp \\
\hline & Comment & $\begin{array}{l}\text { Japan directory : In addition to the appropriate regional documents, the regional xml instance should be located in } \\
\text { this folder. Refer to regional guidance for details }\end{array}$ \\
\hline \multirow{5}{*}{6} & Number & \\
\hline & Title & \\
\hline & Element & \\
\hline & Directory & module-1/us \\
\hline & Comment & $\begin{array}{l}\text { US directory : In addition to the appropriate regional documents, the regional } \mathrm{xml} \text { instance should be located in this } \\
\text { folder. Refer to regional guidance for details }\end{array}$ \\
\hline \multirow[t]{4}{*}{7} & Number & \\
\hline & Title & \\
\hline & Element & \\
\hline & Directory & module-1/xx \\
\hline
\end{tabular}


\begin{tabular}{|l|l|l|}
\hline Comment & $\begin{array}{l}\mathrm{x} \text { directory; where } \mathrm{xx} \text { is a two character country code from ISO-3166-1.: In addition to the appropriate regional } \\
\text { documents, the regional xml instance should be located in this folder. Refer to regional guidance for details }\end{array}$ \\
\hline
\end{tabular} 


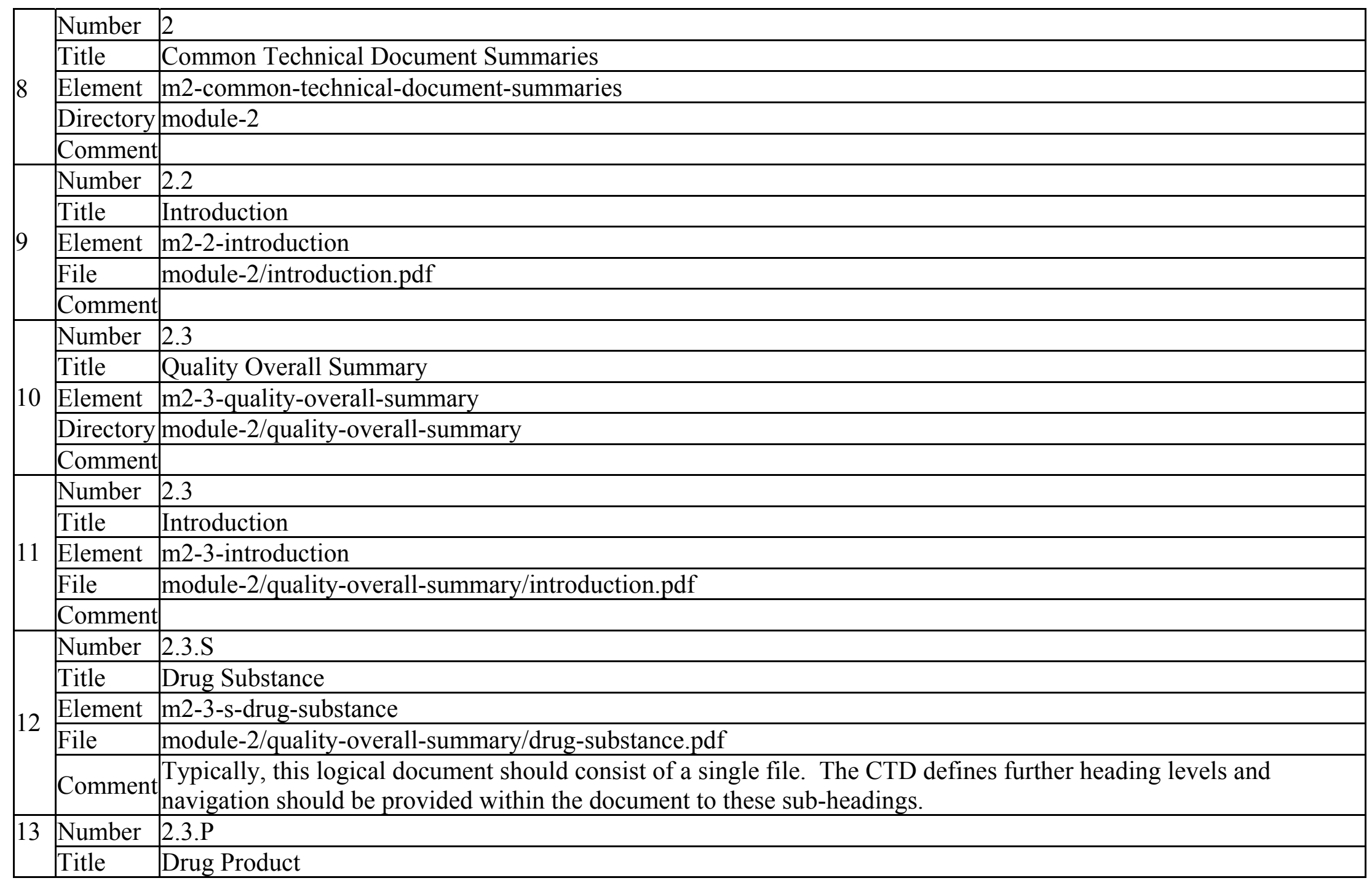




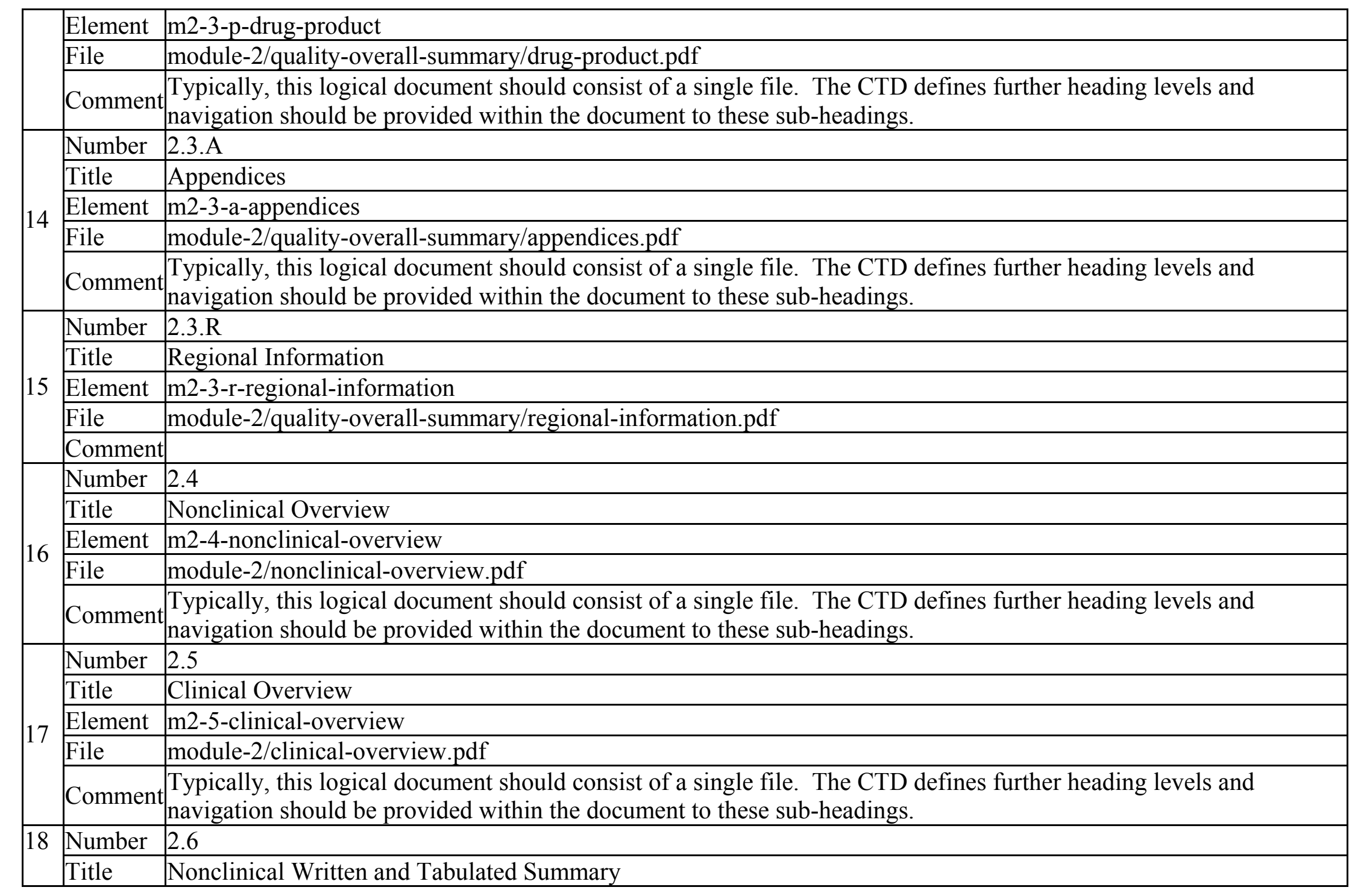




\begin{tabular}{|c|c|c|}
\hline & \multicolumn{2}{|c|}{\begin{tabular}{|l|l} 
Element & m2-6-nonclinical-written-and-tabulated-summary \\
\end{tabular}} \\
\hline & Directory & module-2/nonclinical-summary \\
\hline & Comment & \\
\hline \multirow{5}{*}{19} & Number & 2.6 .1 \\
\hline & Title & Introduction \\
\hline & Element & m2-6-1-introduction \\
\hline & File & module-2/nonclinical-summary/introduction.pdf \\
\hline & Comment & \\
\hline \multirow{5}{*}{20} & Number & 2.6 .2 \\
\hline & Title & Pharmacology Written Summary \\
\hline & Element & m2-6-2-pharmacology-written-summary \\
\hline & File & module-2/nonclinical-summary/pharmacol-written-summary.pdf \\
\hline & Comment & $\begin{array}{l}\text { Typically, this logical document should consist of a single file. The CTD defines further heading levels and } \\
\text { navigation should be provided within the document to these sub-headings. }\end{array}$ \\
\hline \multirow{5}{*}{21} & Number & 2.6 .3 \\
\hline & Title & Pharmacology Tabulated Summary \\
\hline & 1 Element & m2-6-3-pharmacology-tabulated-summary \\
\hline & File & module-2/nonclinical-summary/phamacol-tabulated-summary.pdf \\
\hline & Comment & Should have further navigation via bookmarks \\
\hline \multirow{5}{*}{22} & Number & 2.6 .4 \\
\hline & Title & Pharmacokinetics Written Summary \\
\hline & Element & m2-6-4-pharmacokinetics-written-summary \\
\hline & File & module-2/nonclinical-summary/pharmkin-written-summary.pdf \\
\hline & Comment & $\begin{array}{l}\text { Typically, this logical document should consist of a single file. The CTD defines further heading levels and } \\
\text { navigation should be provided within the document to these sub-headings. }\end{array}$ \\
\hline \multirow[t]{4}{*}{23} & 3 Number & 2.6 .5 \\
\hline & Title & Pharmacokinetics Tabulated Summary \\
\hline & Element & m2-6-5-pharmacokinetics-tabulated-summary \\
\hline & File & module-2/nonclinical-summary/pharmkin-tabulated-summary.pdf \\
\hline
\end{tabular}




\begin{tabular}{|c|c|c|}
\hline & Comment & Should have further navigation via bookmarks \\
\hline \multirow{5}{*}{24} & Number & 2.6 .6 \\
\hline & Title & Toxicology Written Summary \\
\hline & Element & m2-6-6-toxicology-written-summary \\
\hline & File & module-2/nonclinical-summary/toxicology-written-summary.pdf \\
\hline & Comment & $\begin{array}{l}\text { Typically, this logical document should consist of a single file. The CTD defines further heading levels and } \\
\text { navigation should be provided within the document to these sub-headings. }\end{array}$ \\
\hline \multirow{5}{*}{25} & Number & 2.6 .7 \\
\hline & Title & Toxicology Tabulated Summary \\
\hline & Element & m2-6-7-toxicology-tabulated-summary \\
\hline & File & module-2/nonclinical-summary/toxicology-tabulated-summary.pdf \\
\hline & Comment & Should have further navigation via bookmarks \\
\hline \multirow{5}{*}{26} & Number & 2.7 \\
\hline & Title & Clinical Summary \\
\hline & Element & m2-7-clinical-summary \\
\hline & Directory & module-2/clinical-summary \\
\hline & Comment & \\
\hline \multirow{5}{*}{27} & Number & 2.7 .1 \\
\hline & Title & Summary of Biopharmaceutic and Associated Analytical Methods \\
\hline & Element & m2-7-1-summary-of-biopharmaceutic-and-associated-analytical-methods \\
\hline & File & module-2/clinical-summary/summary-biopharm.pdf \\
\hline & Comment & $\begin{array}{l}\text { Typically, this logical document should consist of a single file. The CTD defines further heading levels and } \\
\text { navigation should be provided within the document to these sub-headings. }\end{array}$ \\
\hline \multirow{5}{*}{28} & Number & 2.7 .2 \\
\hline & Title & Summary of Clinical Pharmacology Studies \\
\hline & Element & m2-7-2-summary-of-clinical-pharmacology-studies \\
\hline & File & module-2/clinical-summary/summary-clin-pharm.pdf \\
\hline & Comment & $\begin{array}{l}\text { Typically, this logical document should consist of a single file. The CTD defines further heading levels and } \\
\text { navigation should be provided within the document to these sub-headings. }\end{array}$ \\
\hline
\end{tabular}




\begin{tabular}{|c|c|c|}
\hline \multirow[b]{5}{*}{29} & Number & 2.7 .3 \\
\hline & Title & Summary of Clinical Efficacy - Indication \\
\hline & Element & m2-7-3-summary-of-clinical-efficacy \\
\hline & File & module-2/clinical-summary/summary-clin-efficacy-indication.pdf \\
\hline & Comment & $\begin{array}{l}\text { The folder name should always include the indication being claimed (abbreviated if appropriate) eg. 'summary-clin- } \\
\text { efficacy-asthma'. Where there is more than one indication (eg asthma \& migraine) then the first indication has a } \\
\text { folder 'summary-clin-efficacy-asthma' and the second 'summary-clin-efficacy-migraine'. } \\
\text { Typically, this logical document should consist of a single file. The CTD defines further heading levels and } \\
\text { navigation should be provided within the document to these sub-headings. }\end{array}$ \\
\hline \multirow{5}{*}{30} & Number & 2.7 .4 \\
\hline & Title & Summary of Clinical Safety \\
\hline & Element & m2-7-4-summary-of-clinical-safety \\
\hline & File & module-2/clinical-summary/summary-clin-safety.pdf \\
\hline & Comment & $\begin{array}{l}\text { Typically, this logical document should consist of a single file. The CTD defines further heading levels and } \\
\text { navigation should be provided within the document to these sub-headings. }\end{array}$ \\
\hline \multirow{5}{*}{31} & Number & 2.7 .5 \\
\hline & Title & References \\
\hline & Element & m2-7-5-references \\
\hline & File & module-2/clinical-summary/references.pdf \\
\hline & Comment & \\
\hline \multirow{5}{*}{32} & Number & 2.7 .6 \\
\hline & Title & Synopses of Individual Studies \\
\hline & Element & m2-7-6-synopses-of-individual-studies \\
\hline & File & module-2/clinical-summary/synopses-indiv-studies.pdf \\
\hline & Con & $\begin{array}{l}\text { These synopses should already be located in the Clinical Study Reports in Module } 5 \text { and should not, therefore, be } \\
\text { repeated in Module 2. It is considered sufficient to provide hyperlinks to the locations in Module } 5 \text {. }\end{array}$ \\
\hline
\end{tabular}




\begin{tabular}{|c|c|c|}
\hline & Number & 3 \\
\hline & Title & Quality \\
\hline 33 & Element & m3-quality \\
\hline & Directory & module-3 \\
\hline & Comment & \\
\hline & Number & 3.2 \\
\hline & Title & Body of Data \\
\hline 34 & Element & m3-2-body-of-data \\
\hline & Directory & module-3/body-of-data \\
\hline & Comment & \\
\hline & Number & 3.2.S \\
\hline & Title & Drug Substance \\
\hline 35 & Element & m3-2-s-drug-substance \\
\hline & Directory & module-3/body-of-data/drug-substance \\
\hline & Comment & \\
\hline 36 & Number & $3.2 . \mathrm{S}$ \\
\hline & Title & Drug Substance - Drug Substance Name - Manufacturer \\
\hline & Element & m3-2-s-drug-substance \\
\hline
\end{tabular}




\begin{tabular}{|c|c|c|}
\hline & Comment & $\begin{array}{l}\text { The folder name should always include the name of the drug substance eg. ranitidine through inclusion of the } \\
\text { International Non-proprietary Name to give 'ranitidine-hydrochloride'. Similarly, for manufacturer, the folder name should } \\
\text { always include the name of the manufacturer eg. ranitidine-manufacturer-1. } \\
\text { Where there is more than one manufacturer, the drug substance folder should be repeated but with an indication of each } \\
\text { manufacturer concerned included in the folder name, the first instance eg. 'drug-substance-1- manufacturer-1' and the second } \\
\text { 'drug-substance-1-manufacturer-2'. } \\
\text { Where there is more than one drug substance (eg ranitidine hydrochloride and cimetidine) then the first drug substance } \\
\text { has a folder 'ranitidine-hydrochloride' and the second 'cimetidine'. } \\
\text { In this example a set of folders can include: } \\
\text { ranitidine-manufacturer-1 } \\
\text { ranitidine-manufacturer-2 } \\
\text { cimetidine-manufacturer-1 } \\
\text { cimetidine-manufacturer- } 2 \\
\text { Typically the applicant would include the specific manufacturer(s) (and/or site) in the folder name }\end{array}$ \\
\hline \multirow{5}{*}{37} & Number & 3.2.S.1 \\
\hline & Title & General Information \\
\hline & Element & m3-2-s-1-general-information \\
\hline & Directory & module-3/body-of-data/drug-substance/substance-1-manufacturer-1/general-information \\
\hline & Comment & \\
\hline \multirow{5}{*}{38} & Number & 3.2.S.1.1 \\
\hline & Title & Nomenclature \\
\hline & Element & m3-2-s-1-1-nomenclature \\
\hline & File & module-3/body-of-data/drug-substance/substance-1-manufacturer-1/general-information/nomenclature.pdf \\
\hline & Comment & \\
\hline \multirow[t]{2}{*}{39} & Number & 3.2.S.1.2 \\
\hline & Title & Structure \\
\hline
\end{tabular}




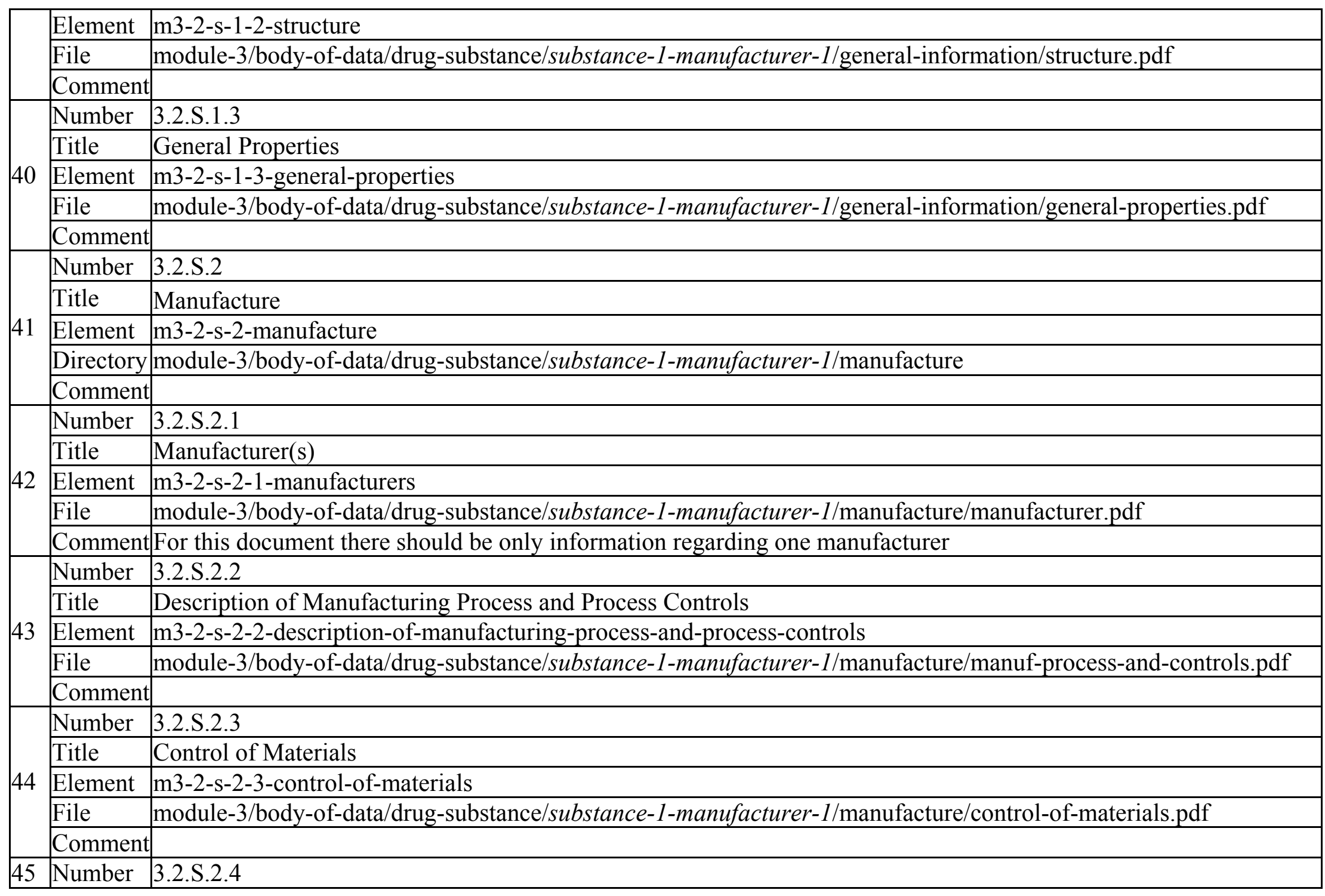

Page 4-12 


\begin{tabular}{|c|c|c|}
\hline & Title & Controls of Critical Steps and Intermediates \\
\hline & Element 1 & m3-2-s-2-4-controls-of-critical-steps-and-intermediates \\
\hline & File & module-3/body-of-data/drug-substance/substance-1-manufacturer-1/manufacture/control-critical-steps.pdf \\
\hline & Comment & \\
\hline & Number & 3.2.S.2.5 \\
\hline & Title & Process Validation and/or Evaluation \\
\hline 46 & 6 Element 1 & m3-2-s-2-5-process-validation-and-or-evaluation \\
\hline & File & module-3/body-of-data/drug-substance/substance-1-manufacturer-1/manufacture/process-validation.pdf \\
\hline & Comment & \\
\hline & Number & 3.2.S.2.6 \\
\hline & Title & Manufacturing Process Development \\
\hline 47 & 7 Element 1 & m3-2-s-2-6-manufacturing-process-development \\
\hline & File & module-3/body-of-data/drug-substance/substance-1-manufacturer-1/manufacture/manuf-process-development.pdf \\
\hline & Comment & \\
\hline & Number & 3.2.S.3 \\
\hline & Title & Characterisation \\
\hline 48 & 8 Element 1 & m3-2-s-3-characterisation \\
\hline & Directory & module-3/body-of-data/drug-substance/substance-1-manufacturer-1/characterisation \\
\hline & Comment & \\
\hline & Number & 3.2.S.3.1 \\
\hline & Title & Elucidation of Structure and Other Characteristics \\
\hline 49 & 9 Element 1 & m3-2-s-3-1-elucidation-of-structure-and-other-characteristics \\
\hline & File & module-3/body-of-data/drug-substance/substance-1-manufacturer-1/characterisation/elucidation-of-structure.pdf \\
\hline & Comment & \\
\hline & Number & 3.2.S.3.2 \\
\hline & Title & Impurities \\
\hline 50 & 0 Element & m3-2-s-3-2-impurities \\
\hline & File & module-3/body-of-data/drug-substance/substance-1-manufacturer-1/characterisation/impurities.pdf \\
\hline & Comment & \\
\hline
\end{tabular}

Page 4-13 


\begin{tabular}{|c|c|c|}
\hline \multirow{5}{*}{51} & Number & 3.2.S.4 \\
\hline & Title & Control of Drug Substance \\
\hline & Element & m3-2-s-4-control-of-drug-substance \\
\hline & Directory & module-3/body-of-data/drug-substance/substance-1-manufacturer-1/control-drug-substance \\
\hline & Comment & \\
\hline \multirow{5}{*}{52} & Number & 3.2.S.4.1 \\
\hline & Title & Specification \\
\hline & Element & m3-2-s-4-1-specification \\
\hline & File & module-3/body-of-data/drug-substance/substance-1-manufacturer-1/control-drug-substance/specification.pdf \\
\hline & Comment & \\
\hline \multirow{5}{*}{53} & Number & 3.2.S.4.2 \\
\hline & Title & Analytical Procedures \\
\hline & Element & m3-2-s-4-2-analytical-procedures \\
\hline & Directory & module-3/body-of-data/drug-substance/substance-1-manufacturer-1/control-drug-substance/analytical-procedures \\
\hline & Comment & For each analytical procedure a separate file should be provided \\
\hline \multirow{5}{*}{54} & Number & 3.2.S.4.2.1 \\
\hline & Title & Analytical Procedure-1 \\
\hline & Element & m3-2-s-4-2-analytical-procedures \\
\hline & File & $\begin{array}{l}\text { module-3/body-of-data/drug-substance/substance-1-manufacturer-1/control-drug-substance/analytical- } \\
\text { procedures/analytical-procedure-1.pdf }\end{array}$ \\
\hline & Comment & \\
\hline \multirow{5}{*}{55} & Number & 3.2.S.4.2.2 \\
\hline & Title & Analytical Procedure-2 \\
\hline & Element & m3-2-s-4-2-analytical-procedures \\
\hline & File & $\begin{array}{l}\text { module-3/body-of-data/drug-substance/substance-1-manufacturer-1/control-drug-substance/analytical- } \\
\text { procedures/analytical-procedure-2.pdf }\end{array}$ \\
\hline & Comment & \\
\hline \multirow[t]{2}{*}{56} & Number & 3.2.S.4.2.3 \\
\hline & Title & Analytical Procedure-3 \\
\hline
\end{tabular}




\begin{tabular}{|c|c|c|}
\hline & \multirow{3}{*}{\begin{tabular}{|l|} 
Element \\
File \\
Comment
\end{tabular}} & m3-2-s-4-2-analytical-procedures \\
\hline & & $\begin{array}{l}\text { module-3/body-of-data/drug-substance/substance-1-manufacturer-1/control-drug-substance/analytical- } \\
\text { procedures/analytical-procedure-3.pdf }\end{array}$ \\
\hline & & \\
\hline \multirow{5}{*}{57} & Number & 3.2.S.4.3 \\
\hline & Title & Validation of Analytical Procedures \\
\hline & Element & m3-2-s-4-3-validation-of-analytical-procedures \\
\hline & Directory & $\begin{array}{l}\text { module-3/body-of-data/drug-substance/substance-1-manufacturer-1/control-drug-substance/validation-analyt- } \\
\text { procedures }\end{array}$ \\
\hline & Comment & For each validation of an analytical procedure a separate file should be provided \\
\hline \multirow{5}{*}{58} & Number & 3.2.S.4.3.1 \\
\hline & Title & Validation of Analytical Procedures \\
\hline & Element & m3-2-s-4-3-validation-of-analytical-procedures \\
\hline & File & $\begin{array}{l}\text { module-3/body-of-data/drug-substance/substance-1-manufacturer-1/control-drug-substance/validation-analyt- } \\
\text { procedures/validation-analyt-procedure-1.pdf }\end{array}$ \\
\hline & Comment & \\
\hline \multirow{5}{*}{59} & Number & 3.2.S.4.3.2 \\
\hline & Title & Validation of Analytical Procedures \\
\hline & Element & m3-2-s-4-3-validation-of-analytical-procedures \\
\hline & File & $\begin{array}{l}\text { module-3/body-of-data/drug-substance/substance-1-manufacturer-1/control-drug-substance/validation-analyt- } \\
\text { procedures/validation-analyt-procedure- } 2 \text {.pdf }\end{array}$ \\
\hline & Comment & \\
\hline \multirow{5}{*}{60} & Number & 3.2.S.4.3.3 \\
\hline & Title & Validation of Analytical Procedures \\
\hline & Element & m3-2-s-4-3-validation-of-analytical-procedures \\
\hline & File & $\begin{array}{l}\text { module-3/body-of-data/drug-substance/substance-1-manufacturer-1/control-drug-substance/validation-analyt- } \\
\text { procedures/validation-analyt-procedure-3.pdf }\end{array}$ \\
\hline & Comment & \\
\hline 61 & Numb & 3.2 .5 \\
\hline
\end{tabular}

Page 4-15 


\begin{tabular}{|c|c|c|}
\hline & Title & Batch Analyses \\
\hline & Element & m3-2-s-4-4-batch-analyses \\
\hline & File & module-3/body-of-data/drug-substance/substance-1-manufacturer-1/control-drug-substance/batch-analyses.pdf \\
\hline & Comment & \\
\hline \multirow{5}{*}{62} & Number & 3.2.S.4.5 \\
\hline & Title & Justification of Specification \\
\hline & Element & m3-2-s-4-5-justification-of-specification \\
\hline & File & $\begin{array}{l}\text { module-3/body-of-data/drug-substance/substance-1-manufacturer-1/control-drug-substance/justification-of- } \\
\text { specification.pdf }\end{array}$ \\
\hline & Comment & \\
\hline \multirow{5}{*}{63} & Number & 3.2. S.5 \\
\hline & Title & Reference Standards or Materials \\
\hline & Element & m3-2-s-5-reference-standards-or-materials \\
\hline & File & module-3/body-of-data/drug-substance/substance-1-manufacturer-1/reference-standards.pdf \\
\hline & Comment & $\begin{array}{l}\text { The applicant can decide whether one file is provided that covers all reference standards or individual files are } \\
\text { tprovided for each reference standard. In deciding whether one or more files are appropriate, it should be considered } \\
\text { that once a particular approach has been adopted, this should be maintained throughout the life of the dossier. }\end{array}$ \\
\hline \multirow{5}{*}{64} & Number & 3.2.S.6 \\
\hline & Title & Container Closure System \\
\hline & Element & m3-2-s-6-container-closure-system \\
\hline & File & module-3/body-of-data/drug-substance/substance-1-manufacturer-1/container-closure-system.pdf \\
\hline & Comment & \\
\hline \multirow{5}{*}{65} & Number & 3.2.S.7 \\
\hline & Title & Stability \\
\hline & Element & m3-2-s-7-stability \\
\hline & Directory & module-3/body-of-data/drug-substance/substance-1-manufacturer-1/stability \\
\hline & Comment & \\
\hline \multirow[t]{2}{*}{66} & Number & 3.2.S.7.1 \\
\hline & Title & Stability Summary and Conclusions \\
\hline
\end{tabular}




\begin{tabular}{|c|c|c|}
\hline \multirow{4}{*}{\multicolumn{2}{|c|}{\begin{tabular}{|l|l|} 
Element & n \\
File & n \\
Comment \\
Number
\end{tabular}}} & m3-2-s-7-1-stability-summary-and-conclusions \\
\hline & & module-3/body-of-data/drug-substance/substance-1-manufacturer-1/stability/stability-summary.pdf \\
\hline & & $\mathrm{t}$ \\
\hline & & 3.2.S.7.2 \\
\hline \multirow{4}{*}{67} & Title & Post-approval Stability Protocol and Stability Commitment \\
\hline & Element & m3-2-s-7-2-post-approval-stability-protocol-and-stability-commitment \\
\hline & File & module-3/body-of-data/drug-substance/substance-1-manufacturer-1/stability/postapproval-stability.pdf \\
\hline & Comment & \\
\hline \multirow{5}{*}{68} & Number & 3.2.S.7.3 \\
\hline & Title & Stability Data \\
\hline & Element & m3-2-s-7-3-stability-data \\
\hline & File & module-3/body-of-data/drug-substance/substance-1-manufacturer-1/stability/stability-data.pdf \\
\hline & Comment & \\
\hline \multirow{5}{*}{69} & Number & 3.2.P \\
\hline & Title & Drug Product \\
\hline & Element & m3-2-p-drug-product \\
\hline & Directory & module-3/body-of-data/drug-product \\
\hline & Comment & \\
\hline \multirow[b]{5}{*}{70} & Number & 3.2.P \\
\hline & Title & Drug Product - Name \\
\hline & Element & m3-2-p-drug-product \\
\hline & Directory & module-3/body-of-data/drug-product/product-1 \\
\hline & Comment & $\begin{array}{l}\text { The folder name should always include the name of the drug product through inclusion of the name of the } \\
\text { form/strength to give eg. 'tablet 5mg'. Where there is more than one drug product (eg powder for reconstitution and } \\
\text { diluent) then the first drug product has a folder 'powder-for-reconstitution' and the second 'diluent'. } \\
\text { Refer to regional guidance for definition of what constitutes a drug product and the acceptability of more than one } \\
\text { drug product in an application. }\end{array}$ \\
\hline \multirow[t]{2}{*}{71} & Number & 3.2.P.1 \\
\hline & Title & Description and Composition of the Drug Product \\
\hline
\end{tabular}




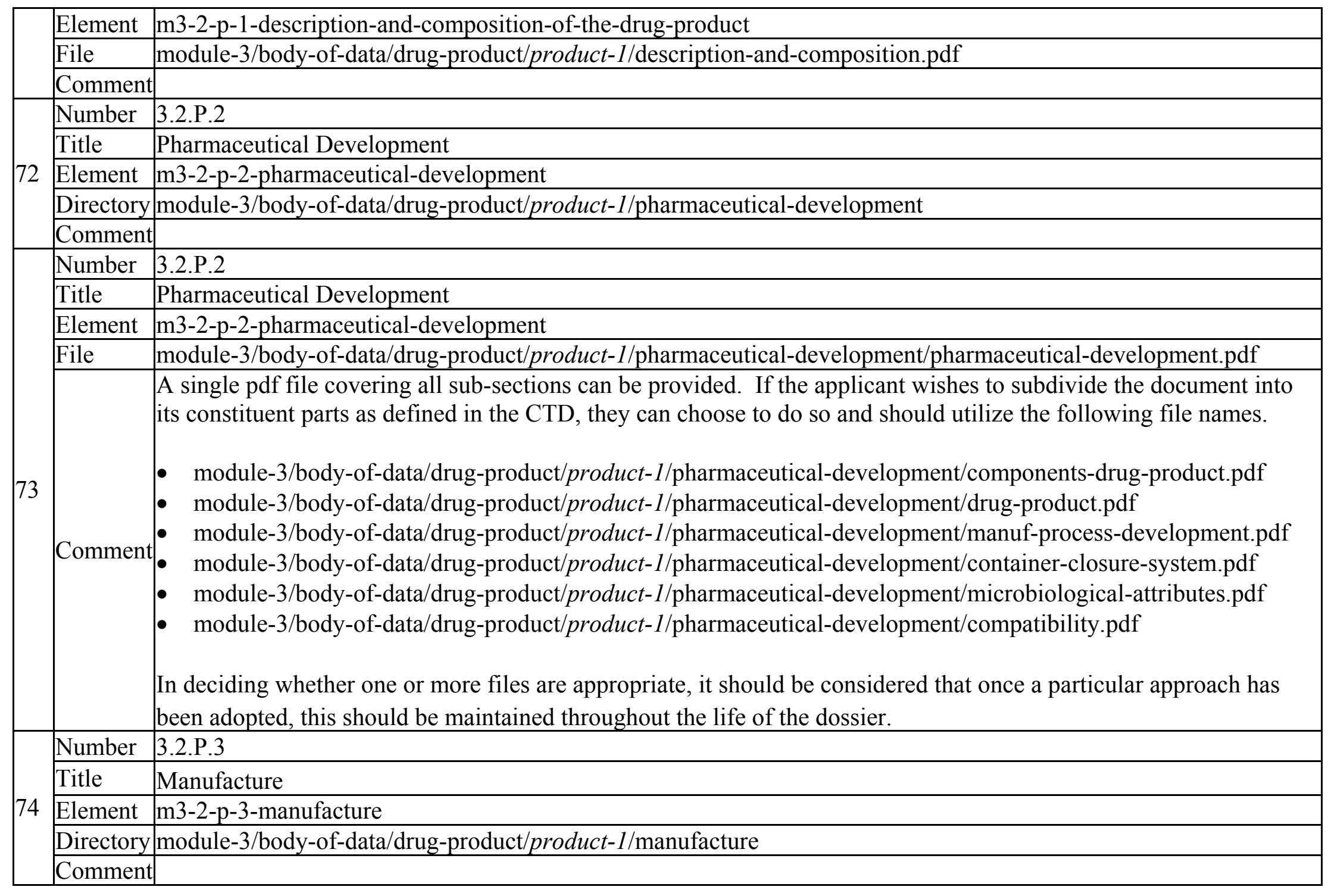

Page 4-18 


\begin{tabular}{|c|c|c|}
\hline \multirow{5}{*}{75} & Number & 3.2.P.3.1 \\
\hline & Title & Manufacturer(s) \\
\hline & Element & m3-2-p-3-1-manufacturers \\
\hline & File & module-3/body-of-data/drug-product/product-1/manufacture/manufacturers.pdf \\
\hline & Comment & \\
\hline \multirow{5}{*}{76} & Number & 3.2.P.3.2 \\
\hline & Title & Batch Formula \\
\hline & Element & m3-2-p-3-2-batch-formula \\
\hline & File & module-3/body-of-data/drug-product/product-1/manufacture/batch-formula.pdf \\
\hline & Comment & \\
\hline \multirow{5}{*}{77} & Number & 3.2.P.3.3 \\
\hline & Title & Description of Manufacturing Process and Process Controls \\
\hline & Element & m3-2-p-3-3-description-of-manufacturing-process-and-process-controls \\
\hline & File & module-3/body-of-data/drug-product/product-1/manufacture/manuf-process-and-controls.pdf \\
\hline & Comment & \\
\hline \multirow{5}{*}{78} & Number & 3.2.P.3.4 \\
\hline & Title & Controls of Critical Steps and Intermediates \\
\hline & Element & m3-2-p-3-4-controls-of-critical-steps-and-intermediates \\
\hline & File & module-3/body-of-data/drug-product/product-1/manufacture/control-critical-steps.pdf \\
\hline & Comment & \\
\hline \multirow{5}{*}{79} & Number & 3.2.P.3.5 \\
\hline & Title & Process Validation and/or Evaluation \\
\hline & Element & m3-2-p-3-5-process-validation-and-or-evaluation \\
\hline & File & module-3/body-of-data/drug-product/product-1/manufacture/process-validation.pdf \\
\hline & Comment & \\
\hline \multirow[t]{4}{*}{80} & Number & 3.2.P. 4 \\
\hline & Title & Control of Excipients \\
\hline & Element & m3-2-p-4-control-of-excipients \\
\hline & Directory & module-3/body-of-data/drug-product/product-1/control-e \\
\hline
\end{tabular}

Page 4-19 


\begin{tabular}{|c|c|c|}
\hline & Comment & \\
\hline \multirow{5}{*}{81} & Number & 3.2.P.4 \\
\hline & Title & Control of Excipients - Excipient \\
\hline & Element & m3-2-p-4-control-of-excipients \\
\hline & Directory & module-3/body-of-data/drug-product/product-1/control-excipients/excipient-1 \\
\hline & Comment & $\begin{array}{l}\text { For a drug product containing more than one excipient, the information requested for sections P4.1 }-\mathrm{P} 4.4 \text { should be } \\
\text { provided in its entirety for each excipient }\end{array}$ \\
\hline \multirow{5}{*}{82} & Number & 3.2.P.4.1 \\
\hline & Title & Specifications \\
\hline & Element & m3-2-p-4-1-specifications \\
\hline & File & module-3/body-of-data/drug-product/product-1/control-excipients/excipient-1/specifications.pdf \\
\hline & Comment & \\
\hline \multirow{5}{*}{83} & Number & 3.2.P.4.2 \\
\hline & Title & Analytical Procedures \\
\hline & Element & m3-2-p-4-2-analytical-procedures \\
\hline & File & module-3/body-of-data/drug-product/product-1/control-excipients/excipient-1/analytical-procedures.pdf \\
\hline & Comment & \\
\hline \multirow{5}{*}{84} & Number & 3.2.P.4.3 \\
\hline & Title & Validation of Analytical Procedures \\
\hline & Element & m3-2-p-4-3-validation-of-analytical-procedures \\
\hline & File & module-3/body-of-data/drug-product/product-1/control-excipients/excipient-1/validation-analyt-procedures.pdf \\
\hline & Comment & \\
\hline \multirow{5}{*}{85} & Number & 3.2.P.4.4 \\
\hline & Title & Justification of Specifications \\
\hline & Element & m3-2-p-4-4-justification-of-specifications \\
\hline & File & module-3/body-of-data/drug-product/product-1/control-excipients/excipient-1/justification-of-specification.pdf \\
\hline & Comment & \\
\hline \multirow[t]{2}{*}{86} & Number & 3.2.P.4.5 \\
\hline & Title & Excipients of Human or Animal Origin \\
\hline
\end{tabular}




\begin{tabular}{|c|c|c|}
\hline & Element & m3-2-p-4-5-excipients-of-human-or-animal-origin \\
\hline & File & module-3/body-of-data/drug-product/product-1/control-excipients/excipients-human-animal.pdf \\
\hline & Comment & \\
\hline & Number & 3.2.P.4.6 \\
\hline & Title & Novel Excipients \\
\hline & 77 Element 1 & m3-2-p-4-6-novel-excipients \\
\hline & File & module-3/body-of-data/drug-product/product-1/control-excipients/novel-excipients.pdf \\
\hline & Comment & \\
\hline & Number & 3.2.P.5 \\
\hline & Title & Control of Drug Product \\
\hline & 8 Element 1 & m3-2-p-5-control-of-drug-product \\
\hline & Directory & module-3/body-of-data/drug-product/product-1/control-drug-product \\
\hline & Comment & \\
\hline & Number & 3.2.P.5.1 \\
\hline & Title & Specification(s) \\
\hline & 9 Element & m3-2-p-5-1-specifications \\
\hline & File & module-3/body-of-data/drug-product/product-1/control-drug-product/specifications.pdf \\
\hline & Comment & \\
\hline & Number & 3.2.P.5.2 \\
\hline & Title & Analytical Procedures \\
\hline & 0 Element & m3-2-p-5-2-analytical-procedures \\
\hline & Directory & module-3/body-of-data/drug-product/product-1/control-drug-product/analytical-procedures \\
\hline & Comment & For each analytical procedure a separate file should be provided \\
\hline & Number & 3.2.P.5.2.1 \\
\hline & Title & Analytical Procedure - 1 \\
\hline & Element & m3-2-p-5-2-analytical-procedures \\
\hline & File & $\begin{array}{l}\text { module-3/body-of-data/drug-product/product-1/control-drug-product/analytical-procedures/analytical-procedure- } \\
\text { 1.pdf }\end{array}$ \\
\hline & Comment & \\
\hline
\end{tabular}

Page 4-21 


\begin{tabular}{|c|c|c|}
\hline \multirow{5}{*}{92} & Number & 3.2.P.5.2.2 \\
\hline & Title & Analytical Procedure -2 \\
\hline & Element & m3-2-p-5-2-analytical-procedures \\
\hline & File & $\begin{array}{l}\text { module-3/body-of-data/drug-product/product-1/control-drug-product/analytical-procedures/analytical-procedure- } \\
\text { 2.pdf }\end{array}$ \\
\hline & Comment & \\
\hline \multirow{5}{*}{93} & Number & 3.2.P.5.2.3 \\
\hline & Title & Analytical Procedure - 3 \\
\hline & Element & m3-2-p-5-2-analytical-procedures \\
\hline & File & $\begin{array}{l}\text { module-3/body-of-data/drug-product/product-1/control-drug-product/analytical-procedures/analytical-procedure- } \\
\text { 3.pdf }\end{array}$ \\
\hline & Comment & \\
\hline \multirow{5}{*}{94} & Number & 3.2.P.5.3 \\
\hline & Title & Validation of Analytical Procedures \\
\hline & Element & m3-2-p-5-3-validation-of-analytical-procedures \\
\hline & Directory & module-3/body-of-data/drug-product/product-1/control-drug-product/validation-analyt-procedures \\
\hline & Comment & For each validation of an analytical procedure, a separate file should be provided \\
\hline \multirow{5}{*}{95} & Number & 3.2.P.5.3.1 \\
\hline & Title & Validation of Analytical Procedures - 1 \\
\hline & Element & m3-2-p-5-3-validation-of-analytical-procedures \\
\hline & File & $\begin{array}{l}\text { module-3/body-of-data/drug-product/product-1/control-drug-product/validation-analyt-procedures/validation- } \\
\text { analytical-procedures-1.pdf }\end{array}$ \\
\hline & Comment & \\
\hline \multirow{5}{*}{96} & Number & 3.2.P.5.3.2 \\
\hline & Title & Validation of Analytical Procedures - 2 \\
\hline & Element & m3-2-p-5-3-validation-of-analytical-procedures \\
\hline & File & $\begin{array}{l}\text { module-3/body-of-data/drug-product/product-1/control-drug-product/validation-analyt-procedures/validation- } \\
\text { analytical-procedures-2.pdf }\end{array}$ \\
\hline & Con & \\
\hline
\end{tabular}

Page 4-22 


\begin{tabular}{|c|c|c|}
\hline \multirow{5}{*}{97} & Number & 3.2.P.5.3.3 \\
\hline & Title & Validation of Analytical Procedures - 3 \\
\hline & Element & m3-2-p-5-3-validation-of-analytical-procedures \\
\hline & File & $\begin{array}{l}\text { module-3/body-of-data/drug-product/product-1/control-drug-product/validation-analyt-procedures/validation- } \\
\text { analytical-procedures-3.pdf }\end{array}$ \\
\hline & Comment & \\
\hline \multirow{5}{*}{98} & Number & 3.2.P.5.4 \\
\hline & Title & Batch Analyses \\
\hline & Element & m3-2-p-5-4-batch-analyses \\
\hline & File & module-3/body-of-data/drug-product/product-1/control-drug-product/batch-analyses.pdf \\
\hline & Comment & \\
\hline \multirow{5}{*}{99} & Number & 3.2.P.5.5 \\
\hline & Title & Characterisation of Impurities \\
\hline & Element & m3-2-p-5-5-characterisation-of-impurities \\
\hline & File & module-3/body-of-data/drug-product/product-1/control-drug-product/characterisation-impurities.pdf \\
\hline & Comment & \\
\hline \multirow{5}{*}{10} & Number & 3.2.P.5.6 \\
\hline & Title & Justification of Specifications \\
\hline & Element & m3-2-p-5-6-justification-of-specifications \\
\hline & File & module-3/body-of-data/drug-product/product-1/control-drug-product/justification-of-specification.pdf \\
\hline & Comment & \\
\hline & Number & 3.2.P.6 \\
\hline & Title & Reference Standards or Materials \\
\hline & Element & m3-2-p-6-reference-standards-or-materials \\
\hline \multirow[t]{2}{*}{101} & File & module-3/body-of-data/drug-product/product-1/reference-standards.pdf \\
\hline & Commer & $\begin{array}{l}\text { The applicant can decide whether one file is provided that covers all reference standards or individual files are } \\
\text { provided for each reference standard. In deciding whether one or more files are appropriate, it should be considered } \\
\text { that once a particular approach has been adopted, this should be maintained throughout the life of the dossier. }\end{array}$ \\
\hline 102 & Number & 3.2.P.7 \\
\hline
\end{tabular}

Page 4-23 


\begin{tabular}{|c|c|c|}
\hline & Title & Container Closure System \\
\hline & Element & m3-2-p-7-container-closure-system \\
\hline & File & module-3/body-of-data/drug-product/product-1/container-closure-system.pdf \\
\hline & Comment & \\
\hline & Number & 3.2.P.8 \\
\hline & Title & Stability \\
\hline 103 & Element & m3-2-p-8-stability \\
\hline & Directory & module-3/body-of-data/drug-product/product-1/stability \\
\hline & Comment & \\
\hline & Number & 3.2.P.8.1 \\
\hline & Title & Stability Summary and Conclusion \\
\hline 104 & Element & m3-2-p-8-1-stability-summary-and-conclusion \\
\hline & File & module-3/body-of-data/drug-product/product-1/stability/stability-summary.pdf \\
\hline & Comment & \\
\hline & Number & 3.2.P.8.2 \\
\hline & Title & Post-approval Stability Protocol and Stability Commitment \\
\hline 105 & Element & m3-2-p-8-2-post-approval-stability-protocol-and-stability-commitment \\
\hline & File & module-3/body-of-data/drug-product/product-1/stability/postapproval-stability.pdf \\
\hline & Comment & \\
\hline & Number & 3.2.P.8.3 \\
\hline & Title & Stability Data \\
\hline 106 & Element & m3-2-p-8-3-stability-data \\
\hline & File & module-3/body-of-data/drug-product/product-1/stability/stability-data.pdf \\
\hline & Comment & \\
\hline & Number & 3.2.A \\
\hline & Title & Appendices \\
\hline 107 & Element & m3-2-a-appendices \\
\hline & Directory & module-3/body-of-data/appendices \\
\hline & Comment & \\
\hline
\end{tabular}

Page 4-24 


\begin{tabular}{|c|c|c|}
\hline \multirow{5}{*}{108} & Number & 3.2.A.1 \\
\hline & Title & Facilities and Equipment \\
\hline & Element & m3-2-a-1-facilities-and-equipment \\
\hline & Directory & module-3/body-of-data/appendices/facilities-and-equipment \\
\hline & Comment & Several reports are likely to be included in this appendix. The organisation is left to the applicant to define \\
\hline \multirow{5}{*}{109} & Number & 3.2.A.1.1 \\
\hline & Title & Facilities and Equipment Report 1 \\
\hline & Element & m3-2-a-1-facilities-and-equipment \\
\hline & File & module-3/body-of-data/appendices/facilities-and-equipment/facilities-and-equipment-report-1.pdf \\
\hline & Comment & \\
\hline \multirow{5}{*}{110} & Number & 3.2.A.1.2 \\
\hline & Title & Facilities and Equipment Report 2 \\
\hline & Element & m3-2-a-1-facilities-and-equipment \\
\hline & File & module-3/body-of-data/appendices/facilities-and-equipment/facilities-and-equipment-report-2.pdf \\
\hline & Comment & \\
\hline \multirow{5}{*}{111} & Number & 3.2.A.1.3 \\
\hline & Title & Facilities and Equipment Report 3 \\
\hline & Element & m3-2-a-1-facilities-and-equipment \\
\hline & File & module-3/body-of-data/appendices/facilities-and-equipment/facilities-and-equipment-report-3.pdf \\
\hline & Comment & \\
\hline \multirow{5}{*}{112} & Number & 3.2.A.2 \\
\hline & Title & Adventitious Agents Safety Evaluation \\
\hline & Element & m3-2-a-2-adventitious-agents-safety-evaluation \\
\hline & Directory & module-3/body-of-data/appendices/adventitious-agents \\
\hline & Comment & $\begin{array}{l}\text { For nonviral adventitious agents reports should be placed in this folder. For viral adventitious agents the following } \\
\text { sub-folder structure should be used. An example of the file naming convention is given for each folder }\end{array}$ \\
\hline \multirow[t]{3}{*}{113} & Number & 3.2.A.2.1 \\
\hline & Title & Adventitious Agents Safety Evaluation Report 1 \\
\hline & Element & m3-2-a-2-adventitious-agents-safety-evaluation \\
\hline
\end{tabular}




\begin{tabular}{|c|c|c|}
\hline & File & module-3/body-of-data/appendices/adventitious-agents/adventitious-agents-report-1.pdf \\
\hline & Comment & \\
\hline & Number & 3.2.A.2.2 \\
\hline & Title & Adventitious Agents Safety Evaluation Report 2 \\
\hline 114 & 4 Element & m3-2-a-2-adventitious-agents-safety-evaluation \\
\hline & File & module-3/body-of-data/appendices/adventitious-agents/adventitious-agents-report-2.pdf \\
\hline & Comment & \\
\hline & Number & 3.2.A.2.3 \\
\hline & Title & Adventitious Agents Safety Evaluation Report 3 \\
\hline 115 & 5 Element & m3-2-a-2-adventitious-agents-safety-evaluation \\
\hline & File & module-3/body-of-data/appendices/adventitious-agents/adventitious-agents-report-3.pdf \\
\hline & Comment & \\
\hline & Number & 3.2.A.3 \\
\hline & Title & Novel Excipients - Name \\
\hline & Element & m3-2-a-3-novel-excipients \\
\hline & Directory & module-3/body-of-data/appendices/novel-excipients-name-1 \\
\hline 116 & & $\begin{array}{l}\text { The name of any novel excipient should be included in the folder name. If there is more than one novel excipient } \\
\text { then each folder should have unique identification through the use of different names eg. 'novel-excipient-name-1' } \\
\text { and 'novel-excipient-name-2. }\end{array}$ \\
\hline & Comment & \\
\hline & & $\begin{array}{l}\text { The directory/file structure would typically follow that of the drug substance section in Module } 3 \text {. Refer to Regional } \\
\text { guidances for the need for such information to be included in the submission directly as opposed to its inclusion in a } \\
\text { Drug Master File. }\end{array}$ \\
\hline & Number & 3.2.R \\
\hline & Title & Regional Information \\
\hline 117 & 7 Element & m3-2-r-regional-information \\
\hline & Directory & module-3/body-of-data/regional-information \\
\hline & Comment & \\
\hline & 8 Number & 3.3 \\
\hline
\end{tabular}

Page 4-26 


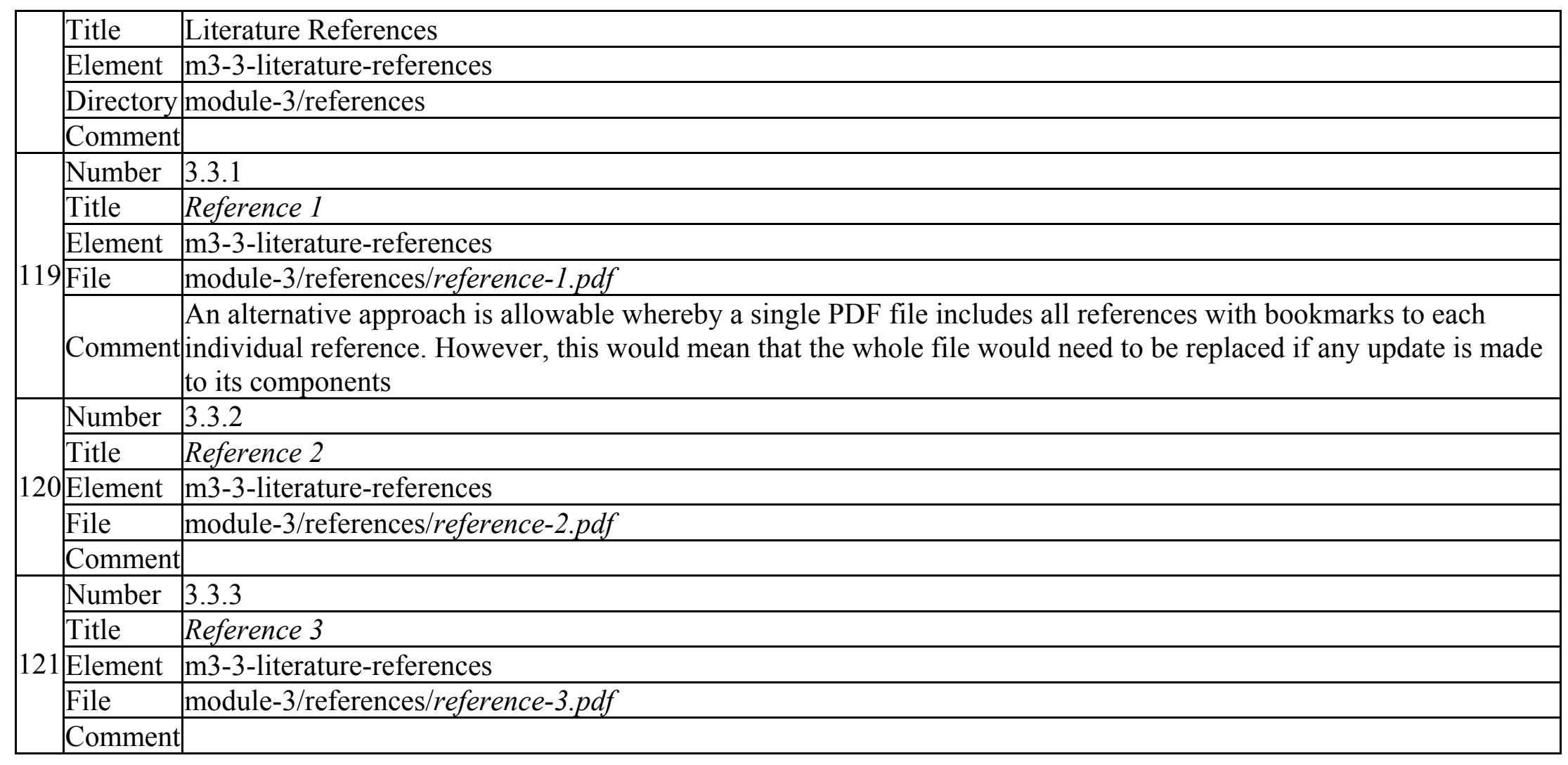




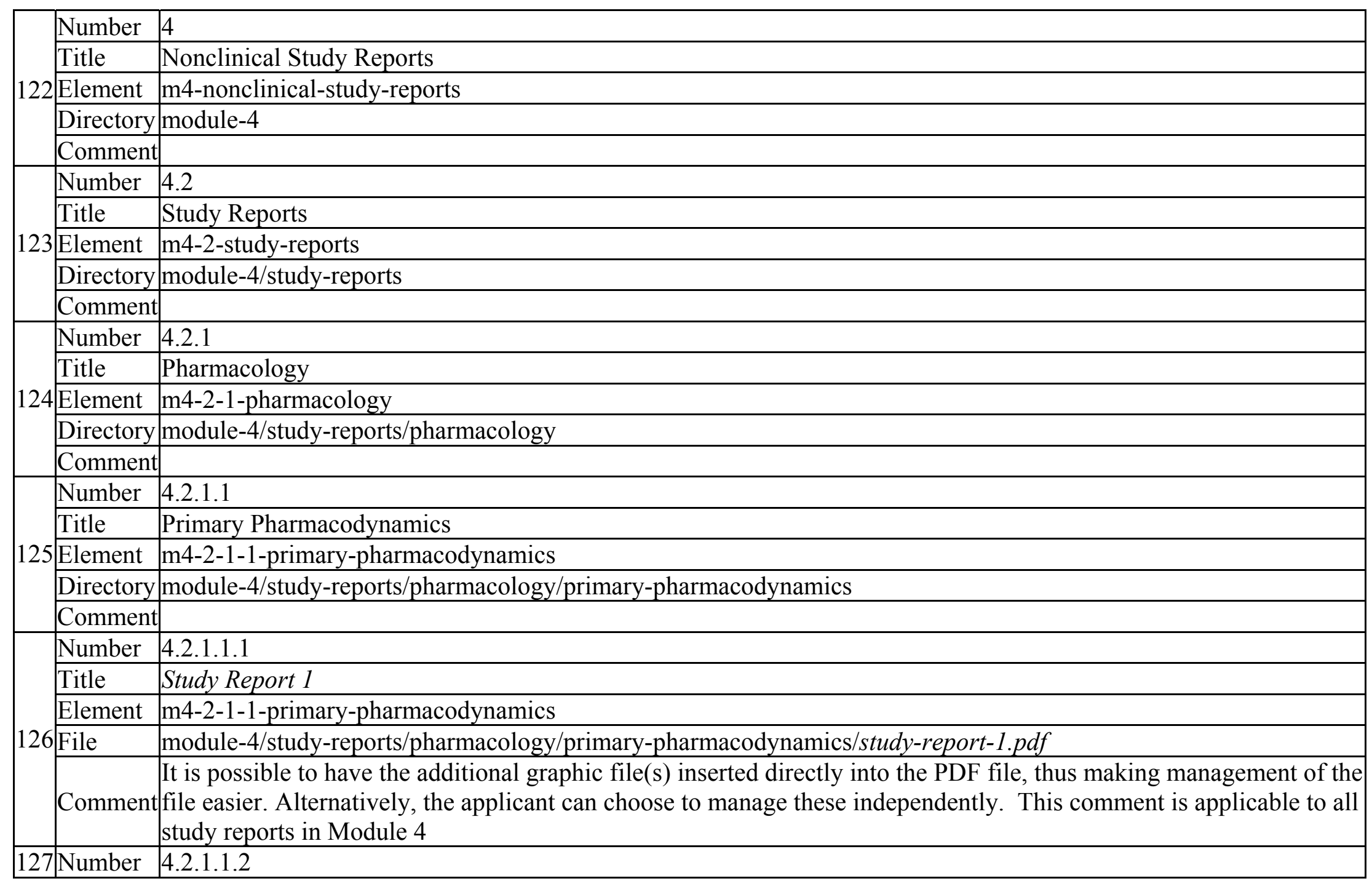

Page 4-28 


\begin{tabular}{|c|c|c|}
\hline & \begin{tabular}{|l|} 
Title \\
Element \\
\end{tabular} & $\begin{array}{l}\text { Study Report } 1 \text { Data } \\
\text { m4-2-1-1-primary-pharmacodynamics }\end{array}$ \\
\hline & File & module-4/study-reports/pharmacology/primary-pharmacodynamics/studv-report-1-data.pdf \\
\hline & Comment & $\begin{array}{l}\text { The data listings can be included as part of the study report document or as a separate appendix. This is relevant to } \\
\text { tall study reports in Module } 4 \text {. Regional requirements can allow the submission of the data listings as a data file. } \\
\text { Refer to regional guidances. }\end{array}$ \\
\hline \multirow{5}{*}{128} & Number & $4.2 \cdot 1.1 .3$ \\
\hline & Title & Study Report 2 \\
\hline & Element & m4-2-1-1-primary-pharmacodynamics \\
\hline & File & module-4/study-reports/pharmacology/primary-pharmacodynamics/study-report-2.pdf \\
\hline & Comment & \\
\hline \multirow{5}{*}{129} & Number & 4.2.1.1.4 \\
\hline & Title & Study Report 2 Data \\
\hline & Element & m4-2-1-1-primary-pharmacodynamics \\
\hline & File & module-4/study-reports/pharmacology/primary-pharmacodynamics/study-report-2-data.pdf \\
\hline & Comment & \\
\hline \multirow{5}{*}{130} & Number & 4.2.1.1.5 \\
\hline & Title & Study Report 3 \\
\hline & Element & m4-2-1-1-primary-pharmacodynamics \\
\hline & File & module-4/study-reports/pharmacology/primary-pharmacodynamics/study-report-3.pdf \\
\hline & Comment & \\
\hline \multirow{5}{*}{131} & Number & 4.2.1.1.6 \\
\hline & Title & Study Report 3 Data \\
\hline & Element & m4-2-1-1-primary-pharmacodynamics \\
\hline & File & module-4/study-reports/pharmacology/primary-pharmacodynamics/study-report-3-data.pdf \\
\hline & Comment & \\
\hline \multirow[t]{3}{*}{132} & Number & 4.2 .1 .2 \\
\hline & Title & Secondary Pharmacodynamics \\
\hline & Element & m4-2-1-2-secondary-pharmacodynamics \\
\hline
\end{tabular}




\begin{tabular}{|c|c|c|}
\hline & \begin{tabular}{|l|l|l} 
Directory \\
Comment
\end{tabular} & module-4/study-reports/pharmacology/secondary-pharmacodynamics \\
\hline & Comment & 4.2 .1 .2 .1 \\
\hline & Title & Study Report 1 \\
\hline 133 & 3 Element & m4-2-1-2-secondary-pharmacodynamics \\
\hline & File & module-4/study-reports/pharmacology/secondary-pharmacodynamics/study-report-1.pdf \\
\hline & Comment & \\
\hline & Number & 4.2.1.2.2 \\
\hline & Title & Study Report 1 Data \\
\hline $134 \mid$ & 4 Element & m4-2-1-2-secondary-pharmacodynamics \\
\hline & File & module-4/study-reports/pharmacology/secondary-pharmacodynamics/study-report-1-data.pdf \\
\hline & Comment & \\
\hline & Number & 4.2.1.2.3 \\
\hline & Title & Study Report 2 \\
\hline 135 & 5 Element & m4-2-1-2-secondary-pharmacodynamics \\
\hline & File & module-4/study-reports/pharmacology/secondary-pharmacodynamics/study-report-2.pdf \\
\hline & Comment & \\
\hline & Number & $4.2 \cdot 1 \cdot 2.4$ \\
\hline & Title & Study Report 2 Data \\
\hline 136 & $6 \longdiv { \text { Element } }$ & m4-2-1-2-secondary-pharmacodynamics \\
\hline & File & module-4/study-reports/pharmacology/secondary-pharmacodynamics/study-report-2-data.pdf \\
\hline & Comment & \\
\hline & Number & $4.2 \cdot 1 \cdot 2.5$ \\
\hline & Title & Study Report 3 \\
\hline 137 & 7 Element & m4-2-1-2-secondary-pharmacodynamics \\
\hline & File & module-4/study-reports/pharmacology/secondary-pharmacodynamics/study-report-3.pdf \\
\hline & Comment & \\
\hline 138 & $8 /$ Number & 4.2.1.2.6 \\
\hline & Title & Study Report 3 Data \\
\hline
\end{tabular}

Page 4-30 


\begin{tabular}{|c|c|c|}
\hline & \begin{tabular}{|l|} 
Element \\
File
\end{tabular} & \begin{tabular}{|l} 
m4-2-1-2-secondary-pharmacodynamics \\
module-4/studv-reports/pharmacologv/secondarv-pharmacodvnamics/studv-report-3-data.pdf
\end{tabular} \\
\hline & Comment & \\
\hline & Number & 4.2 .1 .3 \\
\hline & Title & Safety Pharmacology \\
\hline 139 & Element & m4-2-1-3-safety-pharmacology \\
\hline & Directory & module-4/study-reports/pharmacology/safety-pharmacology \\
\hline & Comment & \\
\hline & Number & 4.2 .1 .3 .1 \\
\hline & Title & Study Report 1 \\
\hline 140 & Element & m4-2-1-3-safety-pharmacology \\
\hline & File & module-4/study-reports/pharmacology/safety-pharmacology/study-report-1.pdf \\
\hline & Comment & \\
\hline & Number & $4.2 \cdot 1 \cdot 3.2$ \\
\hline & Title & Study Report 1 Data \\
\hline 141 & Element & m4-2-1-3-safety-pharmacology \\
\hline & File & module-4/study-reports/pharmacology/safety-pharmacology/study-report-1-data.pdf \\
\hline & Comment & \\
\hline & Number & $4.2 \cdot 1 \cdot 3.3$ \\
\hline & Title & Study Report 2 \\
\hline 142 & Element & m4-2-1-3-safety-pharmacology \\
\hline & File & module-4/study-reports/pharmacology/safety-pharmacology/study-report-2.pdf \\
\hline & Comment & \\
\hline & Number & 4.2.1.3.4 \\
\hline & Title & Study Report 2 Data \\
\hline 143 & Element & m4-2-1-3-safety-pharmacology \\
\hline & File & module-4/study-reports/pharmacology/safety-pharmacology/study-report-2-data.pdf \\
\hline & Comment & \\
\hline & Number & 4.2 .1 .3 .5 \\
\hline
\end{tabular}

Page 4-31 


\begin{tabular}{|c|c|c|}
\hline & Title & Study Report 3 \\
\hline & Element & m4-2-1-3-safety-pharmacology \\
\hline & File & module-4/study-reports/pharmacology/safety-pharmacology/study-report-3.pdf \\
\hline & Commen & \\
\hline & Number & $4.2 \cdot 1 \cdot 3.6$ \\
\hline & Title & Study Report 3 Data \\
\hline 145 & Element & m4-2-1-3-safety-pharmacology \\
\hline & File & module-4/study-reports/pharmacology/safety-pharmacology/study-report-3-data.pdf \\
\hline & Commen & \\
\hline & Number & 4.2 .1 .4 \\
\hline & Title & Pharmacodynamic Drug Interactions \\
\hline 146 & Element & m4-2-1-4-pharmacodynamic-drug-interactions \\
\hline & Directory & module-4/study-reports/pharmacology/pd-drug-interactions \\
\hline & Commen & \\
\hline & Number & 4.2.1.4.1 \\
\hline & Title & Study Report 1 \\
\hline 147 & Element & m4-2-1-4-pharmacodynamic-drug-interactions \\
\hline & File & module-4/study-reports/pharmacology/pd-drug-interactions/study-report-1.pdf \\
\hline & Commen & \\
\hline & Number & 4.2.1.4.2 \\
\hline & Title & Study Report 1 Data \\
\hline 148 & Element & m4-2-1-4-pharmacodynamic-drug-interactions \\
\hline & File & module-4/study-reports/pharmacology/pd-drug-interactions/study-report-1-data.pdf \\
\hline & Commen & \\
\hline & Number & 4.2.1.4.3 \\
\hline & Title & Study Report 2 \\
\hline 149 & Element & m4-2-1-4-pharmacodynamic-drug-interactions \\
\hline & File & module-4/study-reports/pharmacology/pd-drug-interactions/study-report-2.pdf \\
\hline
\end{tabular}

Page 4-32 


\begin{tabular}{|c|c|c|}
\hline & $\begin{array}{l}\text { Number } \\
\text { Title }\end{array}$ & 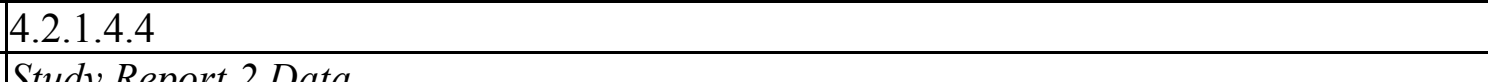 \\
\hline 150 & 0 & 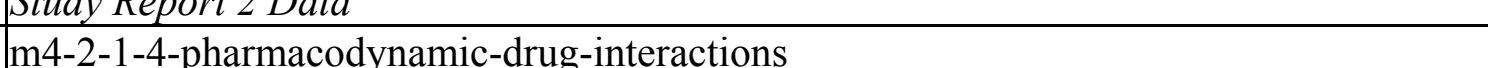 \\
\hline & File & module-4/study-reports/pharmacology/pd-drug-interactions/study-report-2-data.pdf \\
\hline & Comment & \\
\hline & Number & 4.2 .1 .4 .5 \\
\hline & Title & Study Report 3 \\
\hline 151 & $1 \longdiv { \text { Element } }$ & m4-2-1-4-pharmacodynamic-drug-interactions \\
\hline & File & module-4/study-reports/pharmacology/pd-drug-interactions/study-report-3.pdf \\
\hline & Comment & \\
\hline & Number & 4.2 .1 .4 .6 \\
\hline & Title & Study Report 3 Data \\
\hline 152 & 2 Element & m4-2-1-4-pharmacodynamic-drug-interactions \\
\hline & File & module-4/study-reports/pharmacology/pd-drug-interactions/study-report-3-data.pdf \\
\hline & Comment & \\
\hline & Number & 4.2 .2 \\
\hline & Title & Pharmacokinetics \\
\hline 153 & $3 \longdiv { \text { Element } }$ & m4-2-2-pharmacokinetics \\
\hline & Directory & module-4/study-reports/pharmacokinetics \\
\hline & Comment & \\
\hline & Number & 4.2 .2 .1 \\
\hline & Title & Analytical Methods and Validation Reports (if separate reports are available) \\
\hline 154 & $4 \longdiv { \text { Element } }$ & m4-2-2-1-analytical-methods-and-validation-reports \\
\hline & Directory & module-4/study-reports/pharmacokinetics/analyt-methods-validation \\
\hline & Comment & \\
\hline 155 & 5 Number & $4.2 \cdot 2 \cdot 1.1$ \\
\hline & Title & Study Report 1 \\
\hline & Element & m4-2-2-1-analytical-methods-and-validation-reports \\
\hline & File & module-4/study-reports/pharmacokinetics/analyt-methods-validation/study-report-1.p \\
\hline
\end{tabular}

Page 4-33 


\begin{tabular}{|c|c|c|}
\hline & Comment & \\
\hline & Number & 4.2 .2 .1 .2 \\
\hline & Title & Study Report 1 Data \\
\hline 156 & 6 Element & m4-2-2-1-analytical-methods-and-validation-reports \\
\hline & File & module-4/study-reports/pharmacokinetics/analyt-methods-validation/study-report-1-data.pdf \\
\hline & Comment & \\
\hline & Number & 4.2 .2 .1 .3 \\
\hline & Title & Study Report 2 \\
\hline 157 & Element & m4-2-2-1-analytical-methods-and-validation-reports \\
\hline & File & module-4/study-reports/pharmacokinetics/analyt-methods-validation/study-report-2.pdf \\
\hline & Comment & \\
\hline & Number & 4.2 .2 .1 .4 \\
\hline & Title & Study Report 2 Data \\
\hline 158 & 8 Element & m4-2-2-1-analytical-methods-and-validation-reports \\
\hline & File & module-4/study-reports/pharmacokinetics/analyt-methods-validation/study-report-2-data.pdf \\
\hline & Comment & \\
\hline & Number & 4.2 .2 .1 .5 \\
\hline & Title & Study Report 3 \\
\hline 159 & 9 Element & m4-2-2-1-analytical-methods-and-validation-reports \\
\hline & File & module-4/study-reports/pharmacokinetics/analyt-methods-validation/study-report-3.pdf \\
\hline & Comment & \\
\hline & Number & 4.2 .2 .1 .6 \\
\hline & Title & Study Report 3 Data \\
\hline 160 & 0 Element & m4-2-2-1-analytical-methods-and-validation-reports \\
\hline & File & module-4/study-reports/pharmacokinetics/analyt-methods-validation/study-report-3-data.pdf \\
\hline & Comment & \\
\hline 161 & 1 Number & 4.2 .2 .2 \\
\hline & Title & Absorption \\
\hline & Element & m4-2-2-2-absorption \\
\hline
\end{tabular}




\begin{tabular}{|c|c|c|}
\hline & Directory & module-4/study-reports/pharmacokinetics/absorption \\
\hline & Comment & \\
\hline & Number & 4.2.2.2.1 \\
\hline & Title & Study Report 1 \\
\hline 162 & 2 Element & m4-2-2-2-absorption \\
\hline & File & module-4/study-reports/pharmacokinetics/absorption/study-report-1.pdf \\
\hline & Comment & \\
\hline & Number & 4.2 .2 .2 .2 \\
\hline & Title & Study Report 1 Data \\
\hline 163 & 3 Element & m4-2-2-2-absorption \\
\hline & File & module-4/study-reports/pharmacokinetics/absorption/study-report-1-data.pdf \\
\hline & Comment & \\
\hline & Number & $4.2 \cdot 2 \cdot 2.3$ \\
\hline & Title & Study Report 2 \\
\hline 164 & 4 Element & m4-2-2-2-absorption \\
\hline & File & module-4/study-reports/pharmacokinetics/absorption/study-report-2.pdf \\
\hline & Comment & \\
\hline & Number & 4.2 .2 .2 .4 \\
\hline & Title & Study Report 2 Data \\
\hline 165 & 5 Element & m4-2-2-2-absorption \\
\hline & File & module-4/study-reports/pharmacokinetics/absorption/study-report-2-data.pdf \\
\hline & Comment & \\
\hline & Number & 4.2 .2 .2 .5 \\
\hline & Title & Study Report 3 \\
\hline 166 & 6 Element & m4-2-2-2-absorption \\
\hline & File & module-4/study-reports/pharmacokinetics/absorption/study-report-3.pdf \\
\hline & Comment & \\
\hline 167 & 7 Number & 4.2 .2 .2 .6 \\
\hline & Title & Study Report 3 Data \\
\hline
\end{tabular}




\begin{tabular}{|c|c|c|}
\hline & Element & m4-2-2-2-absorption \\
\hline & File & module-4/study-reports/pharmacokinetics/absorption/study-report-3-data.pdf \\
\hline & Comment & \\
\hline & Number & 4.2 .2 .3 \\
\hline & Title & Distribution \\
\hline 168 & Element & m4-2-2-3-distribution \\
\hline & Directory & module-4/study-reports/pharmacokinetics/distribution \\
\hline & Comment & \\
\hline & Number & 4.2.2.3.1 \\
\hline & Title & Study Report 1 \\
\hline 169 & Element & m4-2-2-3-distribution \\
\hline & File & module-4/study-reports/pharmacokinetics/distribution/study-report-1.pdf \\
\hline & Comment & \\
\hline & Number & 4.2.2.3.2 \\
\hline & Title & Study Report 1 Data \\
\hline 170 & Element & m4-2-2-3-distribution \\
\hline & File & module-4/study-reports/pharmacokinetics/distribution/study-report-1-data.pdf \\
\hline & Comment & \\
\hline & Number & 4.2.2.3.3 \\
\hline & Title & Study Report 2 \\
\hline 171 & Element & m4-2-2-3-distribution \\
\hline & File & module-4/study-reports/pharmacokinetics/distribution/study-report-2.pdf \\
\hline & Comment & \\
\hline & Number & 4.2.2.3.4 \\
\hline & Title & Study Report 2 Data \\
\hline 172 & Element & m4-2-2-3-distribution \\
\hline & File & module-4/study-reports/pharmacokinetics/distribution/study-report-2-data.pdf \\
\hline & Comment & \\
\hline 173 & Number & $4.2 \cdot 2 \cdot 3.5$ \\
\hline
\end{tabular}

Page 4-36 


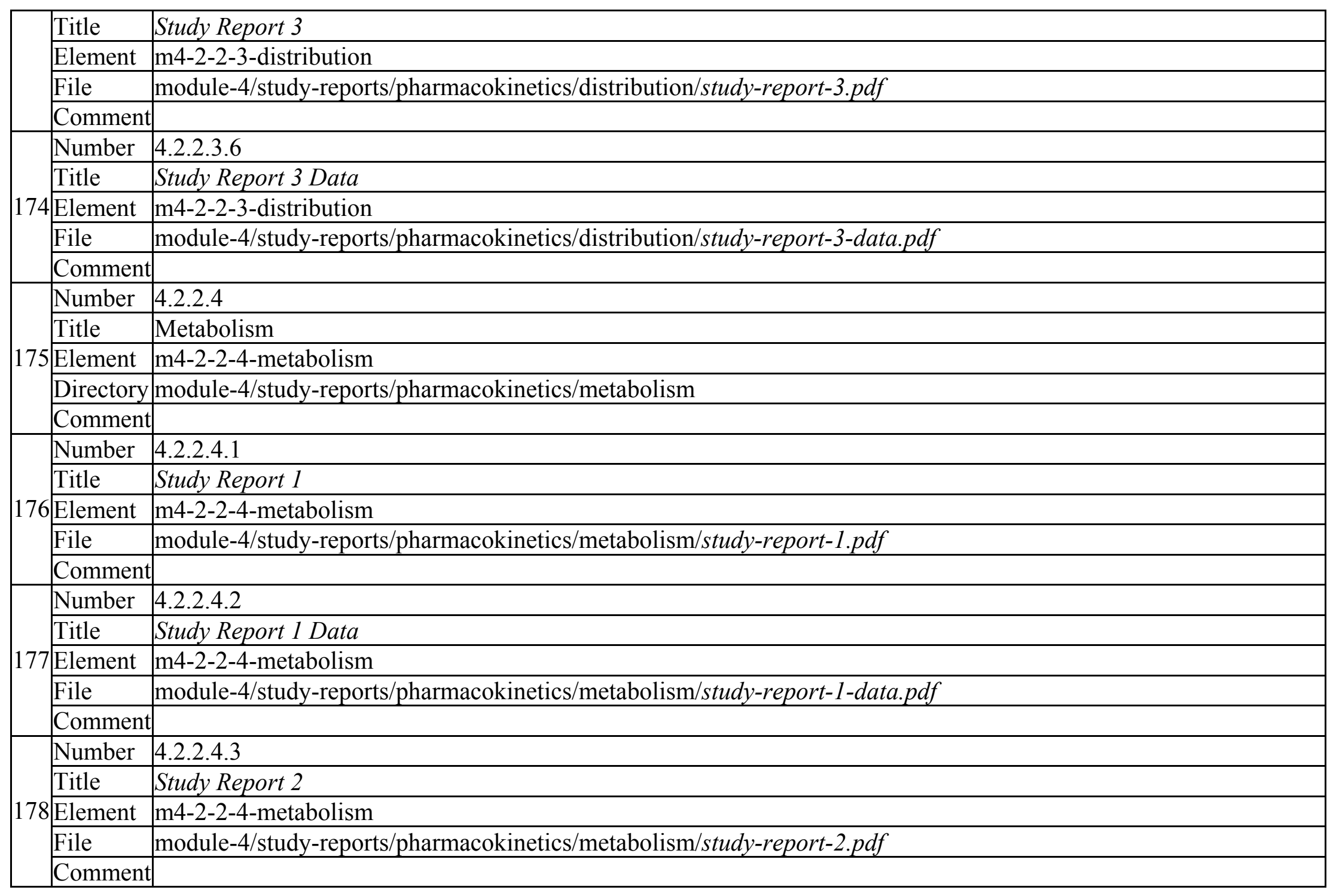




\begin{tabular}{|c|c|c|}
\hline & Number & 4.2.2.4.4 \\
\hline & Title & Study Report 2 Data \\
\hline 179 & Element & m4-2-2-4-metabolism \\
\hline & File & module-4/study-reports/pharmacokinetics/metabolism/study-report-2-data.pdf \\
\hline & Comment & \\
\hline & Number & 4.2 .2 .4 .5 \\
\hline & Title & Study Report 3 \\
\hline 180 & Element & m4-2-2-4-metabolism \\
\hline & File & module-4/study-reports/pharmacokinetics/metabolism/study-report-3.pdf \\
\hline & Comment & \\
\hline & Number & 4.2.2.4.6 \\
\hline & Title & Study Report 3 Data \\
\hline 181 & Element & m4-2-2-4-metabolism \\
\hline & File & module-4/study-reports/pharmacokinetics/metabolism/study-report-3-data.pdf \\
\hline & Comment & \\
\hline & Number & 4.2 .2 .5 \\
\hline & Title & Excretion \\
\hline 182 & Element & m4-2-2-5-excretion \\
\hline & Directory & module-4/study-reports/pharmacokinetics/excretion \\
\hline & Comment & \\
\hline & Number & 4.2 .2 .5 .1 \\
\hline & Title & Study Report 1 \\
\hline 183 & Element & m4-2-2-5-excretion \\
\hline & File & module-4/study-reports/pharmacokinetics/excretion/study-report-1.pdf \\
\hline & Comment & \\
\hline 184 & Number & $4 \cdot 2 \cdot 2 \cdot 5.2$ \\
\hline & Title & Study Report 1 Data \\
\hline & Element & m4-2-2-5-excretion \\
\hline & File & module-4/study-reports/pharmacokinetics/excretion/study-report-1-data.pdf \\
\hline
\end{tabular}




\begin{tabular}{|c|c|c|}
\hline & Comment & \\
\hline & Number & $4.2 \cdot 2 \cdot 5.3$ \\
\hline & Title & Study Report 2 \\
\hline 185 & 5 Element 1 & m4-2-2-5-excretion \\
\hline & File & module-4/study-reports/pharmacokinetics/excretion/study-report-2.pdf \\
\hline & Comment & \\
\hline & Number & $4.2 \cdot 2 \cdot 5.4$ \\
\hline & Title & Study Report 2 Data \\
\hline 186 & 6 Element & m4-2-2-5-excretion \\
\hline & File & module-4/study-reports/pharmacokinetics/excretion/study-report-2-data.pdf \\
\hline & Comment & \\
\hline & Number & $4 \cdot 2 \cdot 2 \cdot 5.5$ \\
\hline & Title & Study Report 3 \\
\hline 187 & 7 Element 1 & m4-2-2-5-excretion \\
\hline & File & module-4/study-reports/pharmacokinetics/excretion/study-report-3.pdf \\
\hline & Comment & \\
\hline & Number & $4 \cdot 2 \cdot 2 \cdot 5.6$ \\
\hline & Title & Study Report 3 Data \\
\hline 188 & 8 Element & m4-2-2-5-excretion \\
\hline & File & module-4/study-reports/pharmacokinetics/excretion/study-report-3-data.pdf \\
\hline & Comment & \\
\hline & Number & 4.2 .2 .6 \\
\hline & Title & Pharmacokinetic Drug Interactions (nonclinical) \\
\hline 189 & 9 Element 1 & m4-2-2-6-pharmacokinetic-drug-interactions \\
\hline & Directory & module-4/study-reports/pharmacokinetics/pk-drug-interactions \\
\hline & Comment & \\
\hline 190 & 0 Number & 4.2.2.6.1 \\
\hline & Title & Study Report 1 \\
\hline & Element 1 & m4-2-2-6-pharmacokinetic-drug-interactions \\
\hline
\end{tabular}




\begin{tabular}{|c|c|c|}
\hline & File & module-4/study-reports/pharmacokinetics/pk-drug-interactions/study-report-1.pdf \\
\hline & Comment & \\
\hline & Number & 4.2.2.6.2 \\
\hline & Title & Study Report 1 Data \\
\hline 191 & 1 Element & m4-2-2-6-pharmacokinetic-drug-interactions \\
\hline & File & module-4/study-reports/pharmacokinetics/pk-drug-interactions/study-report-1-data.pdf \\
\hline & Comment & \\
\hline & Number & $4.2 \cdot 2 \cdot 6.3$ \\
\hline & Title & Study Report 2 \\
\hline 192 & 2 Element & m4-2-2-6-pharmacokinetic-drug-interactions \\
\hline & File & module-4/study-reports/pharmacokinetics/pk-drug-interactions/study-report-2.pdf \\
\hline & Comment & \\
\hline & Number & $4.2 \cdot 2 \cdot 6.4$ \\
\hline & Title & Study Report 2 Data \\
\hline 193 & 3 Element & m4-2-2-6-pharmacokinetic-drug-interactions \\
\hline & File & module-4/study-reports/pharmacokinetics/pk-drug-interactions/study-report-2-data.pdf \\
\hline & Comment & \\
\hline & Number & 4.2 .2 .6 .5 \\
\hline & Title & Study Report 3 \\
\hline 194 & 4 Element & m4-2-2-6-pharmacokinetic-drug-interactions \\
\hline & File & module-4/study-reports/pharmacokinetics/pk-drug-interactions/study-report-3.pdf \\
\hline & Comment & \\
\hline & Number & 4.2.2.6.6 \\
\hline & Title & Study Report 3 Data \\
\hline 195 & 5 Element & m4-2-2-6-pharmacokinetic-drug-interactions \\
\hline & File & module-4/study-reports/pharmacokinetics/pk-drug-interactions/study-report-3-data.pdf \\
\hline & Comment & \\
\hline 196 & 6 Number & $4 \cdot 2.2 .7$ \\
\hline & Title & Other Pharmacokinetic Studies \\
\hline
\end{tabular}




\begin{tabular}{|c|c|c|}
\hline & Element & m4-2-2-7-other-pharmacokinetic-studies \\
\hline & Directory & module-4/study-reports/pharmacokinetics/other-pk-studies \\
\hline & Comment & \\
\hline & Number & 4.2 .2 .7 .1 \\
\hline & Title & Study Report 1 \\
\hline 197 & Element & m4-2-2-7-other-pharmacokinetic-studies \\
\hline & File & module-4/study-reports/pharmacokinetics/other-pk-studies/study-report-1.pdf \\
\hline & Comment & \\
\hline & Number & 4.2 .2 .7 .2 \\
\hline & Title & Study Report 1 Data \\
\hline 198 & Element & m4-2-2-7-other-pharmacokinetic-studies \\
\hline & File & module-4/study-reports/pharmacokinetics/other-pk-studies/study-report-1-data.pdf \\
\hline & Comment & \\
\hline & Number & 4.2 .2 .7 .3 \\
\hline & Title & Study Report 2 \\
\hline 199 & Element & m4-2-2-7-other-pharmacokinetic-studies \\
\hline & File & module-4/study-reports/pharmacokinetics/other-pk-studies/study-report-2.pdf \\
\hline & Comment & \\
\hline & Number & 4.2 .2 .7 .4 \\
\hline & Title & Study Report 2 Data \\
\hline 200 & Element & m4-2-2-7-other-pharmacokinetic-studies \\
\hline & File & module-4/study-reports/pharmacokinetics/other-pk-studies/study-report-2-data.pdf \\
\hline & Comment & \\
\hline & Number & 4.2 .2 .7 .5 \\
\hline & Title & Study Report 3 \\
\hline 201 & Element & m4-2-2-7-other-pharmacokinetic-studies \\
\hline & File & module-4/study-reports/pharmacokinetics/other-pk-studies/study-report-3.pdf \\
\hline & Comment & \\
\hline 202 & Number & 4.2 .2 .7 .6 \\
\hline
\end{tabular}




\begin{tabular}{|l|l|l|}
\hline & Title & Study Report 3 Data \\
\hline Element & m4-2-2-7-other-pharmacokinetic-studies \\
\hline File & module-4/study-reports/pharmacokinetics/other-pk-studies/study-report-3-data.pdf \\
\hline Comment & \\
\hline Number & 4.2 .3 \\
\hline 203 & Title & Toxicology \\
\hline Element & m4-2-3-toxicology \\
\hline Directory & module-4/study-reports/toxicology \\
\hline Comment & \\
\hline Number & 4.2 .3 .1 \\
\hline 204 & Title & Single-Dose Toxicity (in order by species, by route) \\
\hline Element & m4-2-3-1-single-dose-toxicity \\
\hline Directory & module-4/study-reports/toxicology/single-dose-toxicity \\
\hline Comment & \\
\hline Number & 4.2 .3 .1 .1 \\
\hline Title & Study Report 1 \\
\hline 205 & Element & m4-2-3-1-single-dose-toxicity \\
\hline File & module-4/study-reports/toxicology/single-dose-toxicity/study-report-1.pdf \\
\hline Comment & \\
\hline Number & 4.2 .3 .1 .2 \\
\hline Title & Study Report 1 Data \\
\hline 206 & Element & m4-2-3-1-single-dose-toxicity \\
\hline File & module-4/study-reports/toxicology/single-dose-toxicity/study-report-1-data.pdf \\
\hline Comment & \\
\hline Number & 4.2 .3 .1 .3 \\
\hline Title & Study Report 2 \\
\hline 207 & Element & m4-2-3-1-single-dose-toxicity \\
\hline File & module-4/study-reports/toxicology/single-dose-toxicity/study-report-2.pdf \\
\hline Comment & \\
\hline
\end{tabular}

Page 4-42 


\begin{tabular}{|c|c|c|}
\hline & Number & 4.2 .3 .1 .4 \\
\hline & Title & Study Report 2 Data \\
\hline 208 & Element & m4-2-3-1-single-dose-toxicity \\
\hline & File & module-4/study-reports/toxicology/single-dose-toxicity/study-report-2-data.pdf \\
\hline & Comment & \\
\hline & Number & 4.2 .3 .1 .5 \\
\hline & Title & Study Report 3 \\
\hline 209 & Element & m4-2-3-1-single-dose-toxicity \\
\hline & File & module-4/study-reports/toxicology/single-dose-toxicity/study-report-3.pdf \\
\hline & Comment & \\
\hline & Number & 4.2 .3 .1 .6 \\
\hline & Title & Study Report 3 Data \\
\hline 210 & Element & m4-2-3-1-single-dose-toxicity \\
\hline & File & module-4/study-reports/toxicology/single-dose-toxicity/study-report-3-data.pdf \\
\hline & Comment & \\
\hline & Number & 4.2 .3 .2 \\
\hline & Title & Repeat-Dose Toxicity (in order by species, by route, by duration, including supportive toxicokinetics evaluations) \\
\hline 211 & Element & m4-2-3-2-repeat-dose-toxicity \\
\hline & Directory & module-4/study-reports/toxicology/repeat-dose-toxicity \\
\hline & Comment & \\
\hline & Number & 4.2 .3 .2 .1 \\
\hline & Title & Study Report 1 \\
\hline 212 & Element & m4-2-3-2-repeat-dose-toxicity \\
\hline & File & module-4/study-reports/toxicology/repeat-dose-toxicity/study-report-1.pdf \\
\hline & Comment & \\
\hline 213 & Number & 4.2 .3 .2 .2 \\
\hline & Title & Study Report 1 Data \\
\hline & Element & m4-2-3-2-repeat-dose-toxicity \\
\hline & File & module-4/study-reports/toxicology/repeat-dose-toxicity/study-report-1-data.pdf \\
\hline
\end{tabular}




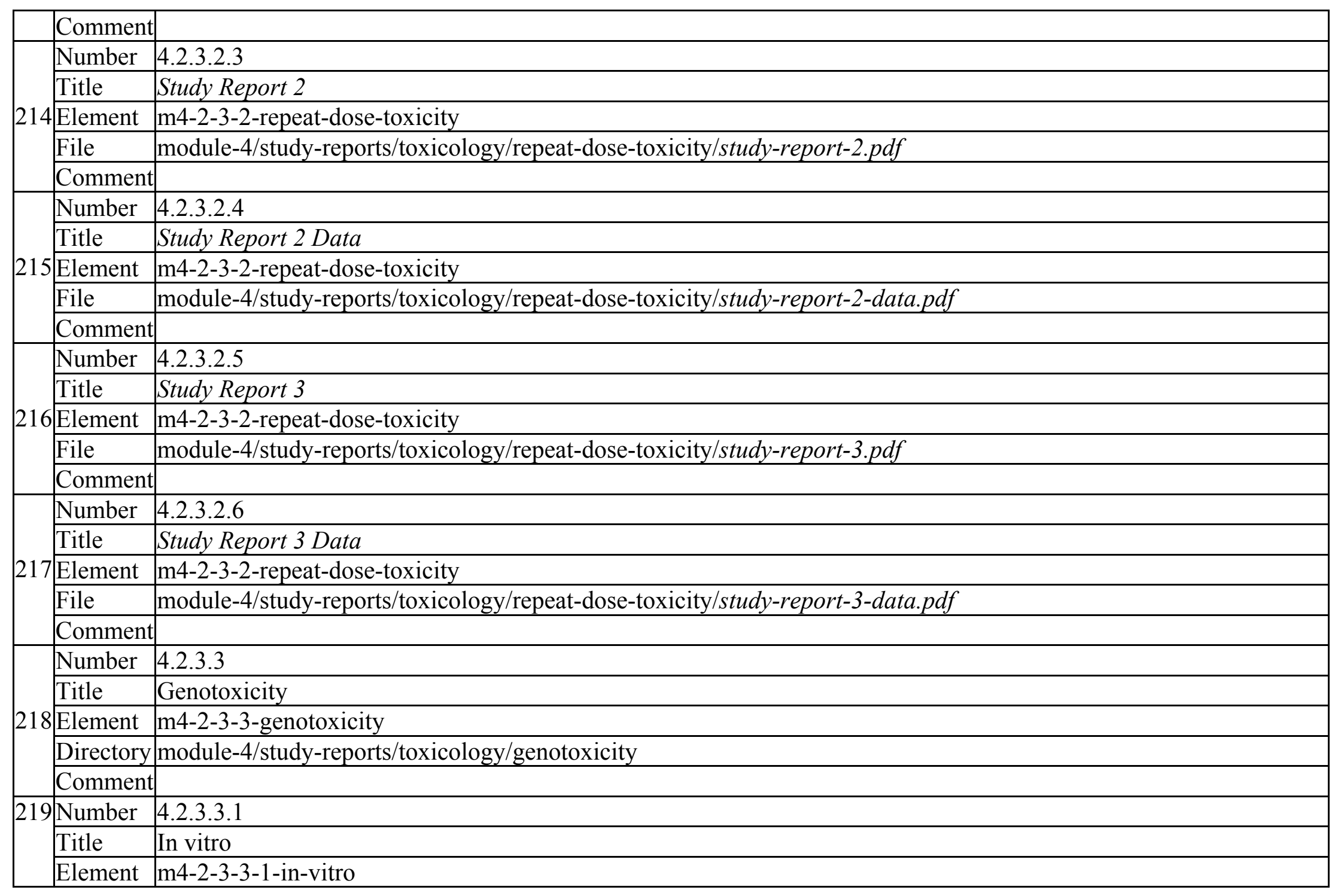




\begin{tabular}{|c|c|c|}
\hline & \begin{tabular}{|l|} 
Directory \\
Comment
\end{tabular} & module-4/study-reports/toxicology/genotoxicity/in-vitro \\
\hline & Number & 4.2 .3 .3 .1 .1 \\
\hline & Title & Study Report 1 \\
\hline 220 & 0 Element & m4-2-3-3-1-in-vitro \\
\hline & File & module-4/study-reports/toxicology/genotoxicity/in-vitro/study-report-1.pdf \\
\hline & Comment & \\
\hline & Number & 4.2 .3 .3 .1 .2 \\
\hline & Title & Study Report 1 Data \\
\hline 221 & 1 Element & m4-2-3-3-1-in-vitro \\
\hline & File & module-4/study-reports/toxicology/genotoxicity/in-vitro/study-report-1-data.pdf \\
\hline & Comment & \\
\hline & Number & 4.2 .3 .3 .1 .3 \\
\hline & Title & Study Report 2 \\
\hline 222 & 2 Element & m4-2-3-3-1-in-vitro \\
\hline & File & module-4/study-reports/toxicology/genotoxicity/in-vitro/study-report-2.pdf \\
\hline & Comment & \\
\hline & Number & 4.2 .3 .3 .1 .4 \\
\hline & Title & Study Report 2 Data \\
\hline 223 & 3 Element & m4-2-3-3-1-in-vitro \\
\hline & File & module-4/study-reports/toxicology/genotoxicity/in-vitro/study-report-2-data.pdf \\
\hline & Comment & \\
\hline & Number & $4.2 .3 \cdot 3 \cdot 1.5$ \\
\hline & Title & Study Report 3 \\
\hline 224 & 4 Element & m4-2-3-3-1-in-vitro \\
\hline & File & module-4/study-reports/toxicology/genotoxicity/in-vitro/study-report-3.pdf \\
\hline & Comment & \\
\hline 225 & 5 Number & 4.2 .3 .3 .1 .6 \\
\hline & Title & Study Report 3 Data \\
\hline
\end{tabular}




\begin{tabular}{|l|l|l|}
\hline Element & m4-2-3-3-1-in-vitro \\
\hline File & module-4/study-reports/toxicology/genotoxicity/in-vitro/study-report-3-data.pdf \\
\hline Comment & \\
\hline Number & 4.2 .3 .3 .2 \\
\hline 226 & Title & In vivo (including supportive toxicokinetics evaluations) \\
\hline Element & m4-2-3-3-2-in-vivo \\
\hline Directory & module-4/study-reports/toxicology/genotoxicity/in-vivo \\
\hline Comment & \\
\hline & Number & 4.2 .3 .3 .2 .1 \\
\hline 227 & Title & Study Report 1 \\
\hline Element & m4-2-3-3-2-in-vivo \\
\hline File & module-4/study-reports/toxicology/genotoxicity/in-vivo/study-report-1.pdf \\
\hline Comment & \\
\hline Number & 4.2 .3 .3 .2 .2 \\
\hline Title & Study Report 1 Data \\
\hline Element & m4-2-3-3-2-in-vivo \\
\hline File & module-4/study-reports/toxicology/genotoxicity/in-vivo/study-report-1-data.pdf \\
\hline Comment & \\
\hline Number & 4.2 .3 .3 .2 .3 \\
\hline Title & Study Report 2 \\
\hline Element & m4-2-3-3-2-in-vivo \\
\hline File & module-4/study-reports/toxicology/genotoxicity/in-vivo/study-report-2.pdf \\
\hline Comment & \\
\hline Number & 4.2 .3 .3 .2 .4 \\
\hline Title & Study Report 2 Data \\
\hline Element & m4-2-3-3-2-in-vivo \\
\hline File & module-4/study-reports/toxicology/genotoxicity/in-vivo/study-report-2-data.pdf \\
\hline Comment & \\
\hline 231 & Number & 4.2 .3 .3 .2 .5 \\
\hline
\end{tabular}

Page 4-46 


\begin{tabular}{|c|c|c|}
\hline & Title & Study Report 3 \\
\hline & Element & m4-2-3-3-2-in-vivo \\
\hline & File & module-4/study-reports/toxicology/genotoxicity/in-vivo/study-report-3.pdf \\
\hline & Comment & \\
\hline & Number & 4.2 .3 .3 .2 .6 \\
\hline & Title & Study Report 3 Data \\
\hline 232 & Element & m4-2-3-3-2-in-vivo \\
\hline & File & module-4/study-reports/toxicology/genotoxicity/in-vivo/study-report-3-data.pdf \\
\hline & Comment & \\
\hline & Number & 4.2 .3 .4 \\
\hline & Title & Carcinogenicity (including supportive toxicokinetics evaluations) \\
\hline 233 & Element & m4-2-3-4-carcinogenicity \\
\hline & Directory & module-4/study-reports/toxicology/carcinogenicity \\
\hline & Comment & \\
\hline & Number & 4.2 .3 .4 .1 \\
\hline 324 & Title & $\begin{array}{l}\text { Long-term studies (in order by species, including range-finding studies that cannot be appropriately included under } \\
\text { repeat-dose toxicity or pharmacokinetics) }\end{array}$ \\
\hline 234 & Element & m4-2-3-4-1-long-term-studies \\
\hline & Directory & module-4/study-reports/toxicology/carcinogenicity/long-term-studies \\
\hline & Comment & \\
\hline & Number & 4.2 .3 .4 .1 .1 \\
\hline & Title & Study Report 1 \\
\hline 235 & Element & m4-2-3-4-1-long-term-studies \\
\hline & File & module-4/study-reports/toxicology/carcinogenicity/long-term-studies/study-report-1.pdf \\
\hline & Comment & \\
\hline 236 & Number & 4.2 .3 .4 .1 .2 \\
\hline & Title & Study Report 1 Data \\
\hline & Element & m4-2-3-4-1-long-term-studies \\
\hline & File & module-4/study-reports/toxicology/carcinogenicity/long-term-studies/study-report-1-data.pdf \\
\hline
\end{tabular}




\begin{tabular}{|c|c|c|}
\hline & Comment & \\
\hline \multirow{5}{*}{237} & Number & 4.2 .3 .4 .1 .3 \\
\hline & Title & Study Report 2 \\
\hline & Element & m4-2-3-4-1-long-term-studies \\
\hline & File & module-4/study-reports/toxicology/carcinogenicity/long-term-studies/study-report-2.pdf \\
\hline & Comment & \\
\hline \multirow{5}{*}{238} & Number & 4.2 .3 .4 .1 .4 \\
\hline & Title & Study Report 2 Data \\
\hline & Element & m4-2-3-4-1-long-term-studies \\
\hline & File & module-4/study-reports/toxicology/carcinogenicity/long-term-studies/study-report-2-data.pdf \\
\hline & Comment & \\
\hline \multirow{5}{*}{239} & Number & 4.2 .3 .4 .1 .5 \\
\hline & Title & Study Report 3 \\
\hline & Element & m4-2-3-4-1-long-term-studies \\
\hline & File & module-4/study-reports/toxicology/carcinogenicity/long-term-studies/study-report-3.pdf \\
\hline & Comment & \\
\hline \multirow{5}{*}{240} & Number & 4.2 .3 .4 .1 .6 \\
\hline & Title & Study Report 3 Data \\
\hline & Element & m4-2-3-4-1-long-term-studies \\
\hline & File & module-4/study-reports/toxicology/carcinogenicity/long-term-studies/study-report-3-data.pdf \\
\hline & Comment & \\
\hline \multirow{5}{*}{241} & Number & 4.2 .3 .4 .2 \\
\hline & Title & $\begin{array}{l}\text { Short- or medium-term studies (including range-finding studies that cannot be appropriately included under repeat- } \\
\text { dose toxicity or pharmacokinetics) }\end{array}$ \\
\hline & Element & m4-2-3-4-2-short-or-medium-term-studies \\
\hline & Directory & module-4/study-reports/toxicology/carcinogenicity/short-medium-term-studies \\
\hline & Comment & \\
\hline \multirow[t]{2}{*}{242} & Number & 4.2 .3 .4 .2 .1 \\
\hline & Title & Study Report 1 \\
\hline
\end{tabular}




\begin{tabular}{|c|c|c|}
\hline & Element & m4-2-3-4-2-short-or-medium-term-studies \\
\hline & File & module-4/study-reports/toxicology/carcinogenicity/short-medium-term-studies/study-report-1.pdf \\
\hline & Comment & \\
\hline & Number & 4.2.3.4.2.2 \\
\hline & Title & Study Report 1 Data \\
\hline 243 & Element & m4-2-3-4-2-short-or-medium-term-studies \\
\hline & File & module-4/study-reports/toxicology/carcinogenicity/short-medium-term-studies/study-report-1-data.pdf \\
\hline & Comment & \\
\hline & Number & 4.2.3.4.2.3 \\
\hline & Title & Study Report 2 \\
\hline 244 & Element & m4-2-3-4-2-short-or-medium-term-studies \\
\hline & File & module-4/study-reports/toxicology/carcinogenicity/short-medium-term-studies/study-report-2.pdf \\
\hline & Comment & \\
\hline & Number & 4.2 .3 .4 .2 .4 \\
\hline & Title & Study Report 2 Data \\
\hline 245 & Element & m4-2-3-4-2-short-or-medium-term-studies \\
\hline & File & module-4/study-reports/toxicology/carcinogenicity/short-medium-term-studies/study-report-2-data.pdf \\
\hline & Comment & \\
\hline & Number & 4.2.3.4.2.5 \\
\hline & Title & Study Report 3 \\
\hline 246 & Element & m4-2-3-4-2-short-or-medium-term-studies \\
\hline & File & module-4/study-reports/toxicology/carcinogenicity/short-medium-term-studies/study-report-3.pdf \\
\hline & Comment & \\
\hline & Number & 4.2.3.4.2.6 \\
\hline & Title & Study Report 3 Data \\
\hline 247 & Element & m4-2-3-4-2-short-or-medium-term-studies \\
\hline & File & module-4/study-reports/toxicology/carcinogenicity/short-medium-term-studies/study-report-3-data.pdf \\
\hline- & Comment & \\
\hline 248 & Number & 4.2 .3 .4 .3 \\
\hline
\end{tabular}




\begin{tabular}{|c|c|c|}
\hline & \begin{tabular}{|l|} 
Title \\
Flement \\
\end{tabular} & Other studies \\
\hline & Directory & module-4/study-reports/toxicology/carcinogenicity/other-studies \\
\hline & Comment & \\
\hline & Number & 4.2.3.4.3.1 \\
\hline & Title & Study Report 1 \\
\hline 249 & Element & m4-2-3-4-3-other-studies \\
\hline & File & module-4/study-reports/toxicology/carcinogenicity/other-studies/study-report-1.pdf \\
\hline & Comment & \\
\hline & Number & 4.2.3.4.3.2 \\
\hline & Title & Study Report 1 Data \\
\hline 250 & Element & m4-2-3-4-3-other-studies \\
\hline & File & module-4/study-reports/toxicology/carcinogenicity/other-studies/study-report-1-data.pdf \\
\hline & Comment & \\
\hline & Number & 4.2.3.4.3.3 \\
\hline & Title & Study Report 2 \\
\hline 251 & Element & m4-2-3-4-3-other-studies \\
\hline & File & module-4/study-reports/toxicology/carcinogenicity/other-studies/study-report-2.pdf \\
\hline & Comment & \\
\hline & Number & 4.2.3.4.3.4 \\
\hline & Title & Study Report 2 Data \\
\hline 252 & Element & m4-2-3-4-3-other-studies \\
\hline & File & module-4/study-reports/toxicology/carcinogenicity/other-studies/study-report-2-data.pdf \\
\hline & Comment & \\
\hline & Number & $4 \cdot 2 \cdot 3 \cdot 4 \cdot 3 \cdot 5$ \\
\hline & Title & Study Report 3 \\
\hline 253 & Element & m4-2-3-4-3-other-studies \\
\hline & File & module-4/study-reports/toxicology/carcinogenicity/other-studies/study-report-3.pdf \\
\hline & Comment & \\
\hline
\end{tabular}




\begin{tabular}{|c|c|c|}
\hline \multirow{4}{*}{254} & \begin{tabular}{|l} 
Number \\
Title
\end{tabular} & \begin{tabular}{|l|} 
4.2.3.4.3.6 \\
Studd, Ponot 3 Data
\end{tabular} \\
\hline & Element & m4-2-3-4-3-other-studies \\
\hline & File & module-4/study-reports/toxicology/carcinogenicity/other-studies/study-report-3-data.pdf \\
\hline & Comment & \\
\hline & Number & 4.2 .3 .5 \\
\hline & Title & $\begin{array}{l}\text { Reproductive and Developmental Toxicity (including range-finding studies and supportive toxicokinetics } \\
\text { evaluations) (If modified study designs are used, the following subheadings should be modified accordingly.) }\end{array}$ \\
\hline & Element & m4-2-3-5-reproductive-and-developmental-toxicity \\
\hline & Directory & module-4/study-reports/toxicology/repro-development-toxicity \\
\hline & Comment & \\
\hline \multirow{5}{*}{256} & Number & 4.2 .3 .5 .1 \\
\hline & Title & Fertility and early embryonic development \\
\hline & Element & m4-2-3-5-1-fertility-and-early-embryonic-development \\
\hline & Directory & module-4/study-reports/toxicology/repro-development-toxicity/fertility-embryonic-develop \\
\hline & Comment & \\
\hline \multirow{5}{*}{257} & Number & 4.2 .3 .5 .1 .1 \\
\hline & Title & Study Report 1 \\
\hline & Element & m4-2-3-5-1-fertility-and-early-embryonic-development \\
\hline & File & module-4/study-reports/toxicology/repro-development-toxicity/fertility-embryonic-develop/study-report-1.pdf \\
\hline & Comment & \\
\hline \multirow{5}{*}{258} & Number & $4.2 .3 \cdot 5 \cdot 1.2$ \\
\hline & Title & Study Report 1 Data \\
\hline & Element & m4-2-3-5-1-fertility-and-early-embryonic-development \\
\hline & File & module-4/study-reports/toxicology/repro-development-toxicity/fertility-embryonic-develop/study-report-1-data.pdf \\
\hline & Comment & \\
\hline \multirow[t]{3}{*}{259} & Number & $4 \cdot 2 \cdot 3 \cdot 5 \cdot 1.3$ \\
\hline & Title & Study Report 2 \\
\hline & Elen & m4-2-3-5-1-fertility-and-early-embryonic-development \\
\hline
\end{tabular}

Page 4-51 


\begin{tabular}{|c|c|c|}
\hline & File & module-4/study-reports/toxicology/repro-development-toxicity/fertility-embryonic-develop/study-report-2.pdf \\
\hline & Comment & \\
\hline & Number & 4.2.3.5.1.4 \\
\hline & Title & Study Report 2 Data \\
\hline 260 & Element & m4-2-3-5-1-fertility-and-early-embryonic-development \\
\hline & File & module-4/study-reports/toxicology/repro-development-toxicity/fertility-embryonic-develop/study-report-2-data.pdf \\
\hline & Comment & \\
\hline & Number & 4.2.3.5.1.5 \\
\hline & Title & Study Report 3 \\
\hline 261 & Element & m4-2-3-5-1-fertility-and-early-embryonic-development \\
\hline & File & module-4/study-reports/toxicology/repro-development-toxicity/fertility-embryonic-develop/study-report-3.pdf \\
\hline & Comment & \\
\hline & Number & 4.2 .3 .5 .1 .6 \\
\hline & Title & Study Report 3 Data \\
\hline 262 & Element & m4-2-3-5-1-fertility-and-early-embryonic-development \\
\hline & File & module-4/study-reports/toxicology/repro-development-toxicity/fertility-embryonic-develop/study-report-3-data.pdf \\
\hline & Comment & \\
\hline & Number & 4.2 .3 .5 .2 \\
\hline & Title & Embryo-fetal development \\
\hline 263 & Element & m4-2-3-5-2-embryo-fetal-development \\
\hline & Directory & module-4/study-reports/toxicology/repro-development-toxicity/embryo-fetal-develop \\
\hline & Comment & \\
\hline & Number & 4.2.3.5.2.1 \\
\hline & Title & Study Report 1 \\
\hline 264 & Element & m4-2-3-5-2-embryo-fetal-development \\
\hline & File & module-4/study-reports/toxicology/repro-development-toxicity/embryo-fetal-develop/study-report-1.pdf \\
\hline & Comment & \\
\hline 265 & Number & 4.2.3.5.2.2 \\
\hline & Title & Study Report 1 Data \\
\hline
\end{tabular}

Page 4-52 


\begin{tabular}{|c|c|c|}
\hline & \begin{tabular}{|l|l|l} 
Element \\
File
\end{tabular} & \begin{tabular}{|l} 
m4-2-3-5-2-embryo-fetal-development \\
module-4/study-reports/toxicology/repro-development-toxicity/embryo-fetal-develop/studv-report-1-data.pdf
\end{tabular} \\
\hline & Comment & \\
\hline & Number & $4.2 .3 \cdot 5 \cdot 2.3$ \\
\hline & Title & Study Report 2 \\
\hline 266 & Element & m4-2-3-5-2-embryo-fetal-development \\
\hline & File & module-4/study-reports/toxicology/repro-development-toxicity/embryo-fetal-develop/study-report-2.pdf \\
\hline & Comment & \\
\hline & Number & $4 \cdot 2 \cdot 3 \cdot 5 \cdot 2.4$ \\
\hline & Title & Study Report 2 Data \\
\hline 267 & Element & m4-2-3-5-2-embryo-fetal-development \\
\hline & File & module-4/study-reports/toxicology/repro-development-toxicity/embryo-fetal-develop/study-report-2-data.pdf \\
\hline & Comment & \\
\hline & Number & $4 \cdot 2 \cdot 3 \cdot 5 \cdot 2.5$ \\
\hline & Title & Study Report 3 \\
\hline 268 & Element & m4-2-3-5-2-embryo-fetal-development \\
\hline & File & module-4/study-reports/toxicology/repro-development-toxicity/embryo-fetal-develop/study-report-3.pdf \\
\hline & Comment & \\
\hline & Number & 4.2 .3 .5 .2 .6 \\
\hline & Title & Study Report 3 Data \\
\hline 269 & Element & m4-2-3-5-2-embryo-fetal-development \\
\hline & File & module-4/study-reports/toxicology/repro-development-toxicity/embryo-fetal-develop/study-report-3-data.pdf \\
\hline & Comment & \\
\hline & Number & 4.2.3.5.3 \\
\hline & Title & Prenatal and postnatal development, including maternal function \\
\hline 270 & Element & m4-2-3-5-3-prenatal-and-postnatal-development-including-maternal-function \\
\hline & Directory & module-4/study-reports/toxicology/repro-development-toxicity/pre-postnatal-develop \\
\hline & Comment & \\
\hline 6 & Number & $4.2 \cdot 3 \cdot 5 \cdot 3.1$ \\
\hline
\end{tabular}

Page 4-53 


\begin{tabular}{|c|c|c|}
\hline & \begin{tabular}{|l|} 
Title \\
Element
\end{tabular} & \begin{tabular}{|l} 
Study Report 1 \\
m4-2-3-5-3-prenatal-and-postnatal-development-including-maternal-function
\end{tabular} \\
\hline & \begin{tabular}{|l|l|l} 
Element \\
File
\end{tabular} & module-4/study-reports/toxicology/repro-development-toxicity/pre-postnatal-develop/studv-report-1.pdf \\
\hline & Comment & \\
\hline & Number & $4 \cdot 2 \cdot 3 \cdot 5 \cdot 3 \cdot 2$ \\
\hline & Title & Study Report 1 Data \\
\hline 272 & Element & m4-2-3-5-3-prenatal-and-postnatal-development-including-maternal-function \\
\hline & File & module-4/study-reports/toxicology/repro-development-toxicity/pre-postnatal-develop/study-report-1-data.pdf \\
\hline & Comment & \\
\hline & Number & $4 \cdot 2 \cdot 3 \cdot 5 \cdot 3.3$ \\
\hline & Title & Study Report 2 \\
\hline 273 & Element & m4-2-3-5-3-prenatal-and-postnatal-development-including-maternal-function \\
\hline & File & module-4/study-reports/toxicology/repro-development-toxicity/pre-postnatal-develop/study-report-2.pdf \\
\hline & Comment & \\
\hline & Number & $4 \cdot 2 \cdot 3 \cdot 5 \cdot 3 \cdot 4$ \\
\hline & Title & Study Report 2 Data \\
\hline 274 & Element & m4-2-3-5-3-prenatal-and-postnatal-development-including-maternal-function \\
\hline & File & module-4/study-reports/toxicology/repro-development-toxicity/pre-postnatal-develop/study-report-2-data.pdf \\
\hline & Comment & \\
\hline & Number & $4 \cdot 2 \cdot 3 \cdot 5 \cdot 3 \cdot 5$ \\
\hline & Title & Study Report 3 \\
\hline 275 & Element & m4-2-3-5-3-prenatal-and-postnatal-development-including-maternal-function \\
\hline & File & module-4/study-reports/toxicology/repro-development-toxicity/pre-postnatal-develop/study-report-3.pdf \\
\hline & Comment & \\
\hline & Number & $4 \cdot 2 \cdot 3 \cdot 5 \cdot 3.6$ \\
\hline & Title & Study Report 3 Data \\
\hline 276 & Element & m4-2-3-5-3-prenatal-and-postnatal-development-including-maternal-function \\
\hline & File & module-4/study-reports/toxicology/repro-development-toxicity/pre-postnatal-develop/study-report-3-data.pdf \\
\hline & Comment & \\
\hline
\end{tabular}

Page 4-54 


\begin{tabular}{|c|c|c|}
\hline & $\begin{array}{ll}\text { Number } \\
\text { Title }\end{array}$ & $\begin{array}{l}4 \cdot 2 \cdot 3 \cdot 5 \cdot 4 \\
\text { Studies in which the offenring (iuvenile animale) are doced and/ar further ovaluated }\end{array}$ \\
\hline 277 & Elitle & m4-2-3-5-4-studies-in-which-the-offspring-juvenile-animals-are-dosed-and-or-further-evaluated \\
\hline & Directory & module-4/study-reports/toxicology/repro-development-toxicity/juvenile \\
\hline & Comment & \\
\hline & Number & 4.2 .3 .5 .4 .1 \\
\hline & Title & Study Report 1 \\
\hline 278 & Element & m4-2-3-5-4-studies-in-which-the-offspring-juvenile-animals-are-dosed-and-or-further-evaluated \\
\hline & File & module-4/study-reports/toxicology/repro-development-toxicity/juvenile/study-report-1.pdf \\
\hline & Comment & \\
\hline & Number & $4 \cdot 2 \cdot 3 \cdot 5 \cdot 4.2$ \\
\hline & Title & Study Report 1 Data \\
\hline 279 & Element & m4-2-3-5-4-studies-in-which-the-offspring-juvenile-animals-are-dosed-and-or-further-evaluated \\
\hline & File & module-4/study-reports/toxicology/repro-development-toxicity/juvenile/study-report-1-data.pdf \\
\hline & Comment & \\
\hline & Number & $4 \cdot 2 \cdot 3 \cdot 5.4 .3$ \\
\hline & Title & Study Report 2 \\
\hline 280 & Element & m4-2-3-5-4-studies-in-which-the-offspring-juvenile-animals-are-dosed-and-or-further-evaluated \\
\hline & File & module-4/study-reports/toxicology/repro-development-toxicity/juvenile/study-report-2.pdf \\
\hline & Comment & \\
\hline & Number & 4.2 .3 .5 .4 .4 \\
\hline & Title & Study Report 2 Data \\
\hline 281 & Element & m4-2-3-5-4-studies-in-which-the-offspring-juvenile-animals-are-dosed-and-or-further-evaluated \\
\hline & File & module-4/study-reports/toxicology/repro-development-toxicity/juvenile/study-report-2-data.pdf \\
\hline & Comment & \\
\hline 282 & Number & 4.2 .3 .5 .4 .5 \\
\hline & Title & Study Report 3 \\
\hline & Element & m4-2-3-5-4-studies-in-which-the-offspring-juvenile-animals-are-dosed-and-or-further-evaluated \\
\hline & File & module-4/study-reports/toxicology/repro-development-toxicity/juvenile/study-report-3.pdf \\
\hline
\end{tabular}

Page 4-55 


\begin{tabular}{|c|c|c|}
\hline & Comment & \\
\hline \multirow{5}{*}{28} & Number & $4 \cdot 2.3 \cdot 5.4 .6$ \\
\hline & Title & Study Report 3 Data \\
\hline & Element & m4-2-3-5-4-studies-in-which-the-offspring-juvenile-animals-are-dosed-and-or-further-evaluated \\
\hline & File & module-4/study-reports/toxicology/repro-development-toxicity/juvenile/study-report-3-data.pdf \\
\hline & Comment & \\
\hline \multirow{5}{*}{284} & Number & 4.2 .4 \\
\hline & Title & Local Tolerance \\
\hline & Element & m4-2-4-local-tolerance \\
\hline & Directory & module-4/study-reports/local-tolerance \\
\hline & Comment & \\
\hline \multirow{5}{*}{285} & Number & 4.2 .4 .1 \\
\hline & Title & Study Report 1 \\
\hline & Element & m4-2-4-local-tolerance \\
\hline & File & module-4/study-reports/local-tolerance/study-report-1.pdf \\
\hline & Comment & \\
\hline \multirow{5}{*}{286} & Number & 4.2 .4 .2 \\
\hline & Title & Study Report 1 Data \\
\hline & Element & m4-2-4-local-tolerance \\
\hline & File & module-4/study-reports/local-tolerance/study-report-1-data.pdf \\
\hline & Comment & \\
\hline \multirow{5}{*}{287} & Number & 4.2 .4 .3 \\
\hline & Title & Study Report 2 \\
\hline & Element & m4-2-4-local-tolerance \\
\hline & File & module-4/study-reports/local-tolerance/study-report-2.pdf \\
\hline & Comment & \\
\hline \multirow[t]{3}{*}{288} & Number & 4.2.4.4 \\
\hline & Title & Study Report 2 Data \\
\hline & Element & m4-2-4-local-tolerance \\
\hline
\end{tabular}




\begin{tabular}{|c|c|c|}
\hline & File & module-4/study-reports/local-tolerance/study-report-2-data.pdf \\
\hline & Comment & \\
\hline & Number & 4.2.4.5 \\
\hline & Title & Study Report 3 \\
\hline 289 & Element & m4-2-4-local-tolerance \\
\hline & File & module-4/study-reports/local-tolerance/study-report-3.pdf \\
\hline & Comment & \\
\hline & Number & 4.2 .4 .6 \\
\hline & Title & Study Report 3 Data \\
\hline 290 & Element & m4-2-4-local-tolerance \\
\hline & File & module-4/study-reports/local-tolerance/study-report-3-data.pdf \\
\hline & Comment & \\
\hline & Number & 4.2 .5 \\
\hline & Title & Other Toxicity Studies (if available) \\
\hline $291 \mid$ & Element & m4-2-5-other-toxicity-studies \\
\hline & Directory & module-4/study-reports/other-toxicity-studies \\
\hline & Comment & \\
\hline & Number & 4.2 .5 .1 \\
\hline & Title & Antigenicity \\
\hline 292 & Element & m4-2-5-1-antigenicity \\
\hline & Directory & module-4/study-reports/other-toxicity-studies/antigenicity \\
\hline & Comment & \\
\hline & Number & 4.2 .5 .1 .1 \\
\hline & Title & Study Report 1 \\
\hline 293 & Element & m4-2-5-1-antigenicity \\
\hline & File & module-4/study-reports/other-toxicity-studies/antigenicity/study-report-1.pdf \\
\hline & Comment & \\
\hline 294 & Number & $4 \cdot 2 \cdot 5 \cdot 1.2$ \\
\hline & Title & Study Report 1 Data \\
\hline
\end{tabular}




\begin{tabular}{|c|c|c|}
\hline & Element & m4-2-5-1-antigenicity \\
\hline & File & module-4/study-reports/other-toxicity-studies/antigenicity/study-report-1-data.pdf \\
\hline & Comment & \\
\hline & Number & 4.2 .5 .1 .3 \\
\hline & Title & Study Report 2 \\
\hline 295 & Element & m4-2-5-1-antigenicity \\
\hline & File & module-4/study-reports/other-toxicity-studies/antigenicity/study-report-2.pdf \\
\hline & Comment & \\
\hline & Number & 4.2 .5 .1 .4 \\
\hline & Title & Study Report 2 Data \\
\hline 296 & Element & m4-2-5-1-antigenicity \\
\hline & File & module-4/study-reports/other-toxicity-studies/antigenicity/study-report-2-data.pdf \\
\hline & Comment & \\
\hline & Number & 4.2 .5 .1 .5 \\
\hline & Title & Study Report 3 \\
\hline 297 & Element & m4-2-5-1-antigenicity \\
\hline & File & module-4/study-reports/other-toxicity-studies/antigenicity/study-report-3.pdf \\
\hline & Comment & \\
\hline & Number & 4.2 .5 .1 .6 \\
\hline & Title & Study Report 3 Data \\
\hline 298 & Element & m4-2-5-1-antigenicity \\
\hline & File & module-4/study-reports/other-toxicity-studies/antigenicity/study-report-3-data.pdf \\
\hline & Comment & \\
\hline & Number & 4.2 .5 .2 \\
\hline & Title & Immunotoxicity \\
\hline 299 & Element & m4-2-5-2-immunotoxicity \\
\hline & Directory & module-4/study-reports/other-toxicity-studies/immunotoxicity \\
\hline & Comment & \\
\hline 300 & Number & 4.2 .5 .2 .1 \\
\hline
\end{tabular}




\begin{tabular}{|c|c|c|}
\hline & \begin{tabular}{|l|} 
Title \\
Element \\
\end{tabular} & $\begin{array}{l}\text { Study Report } 1 \\
\text { m4-2-5-2-immunotoxicity }\end{array}$ \\
\hline & \begin{tabular}{|l|} 
Element \\
File
\end{tabular} & module-4/studv-reports/other-toxicitv-studies/immunotoxicitv/studv-report-1 pdf \\
\hline & Comment & \\
\hline & Number & $4.2 .5 \cdot 2.2$ \\
\hline & Title & Study Report 1 Data \\
\hline 301 & Element & m4-2-5-2-immunotoxicity \\
\hline & File & module-4/study-reports/other-toxicity-studies/immunotoxicity/study-report-1-data.pdf \\
\hline & Comment & \\
\hline & Number & $4.2 .5 \cdot 2.3$ \\
\hline & Title & Study Report 2 \\
\hline 302 & Element & m4-2-5-2-immunotoxicity \\
\hline & File & module-4/study-reports/other-toxicity-studies/immunotoxicity/study-report-2.pdf \\
\hline & Comment & \\
\hline & Number & $4.2 .5 \cdot 2.4$ \\
\hline & Title & Study Report 2 Data \\
\hline 303 & Element & m4-2-5-2-immunotoxicity \\
\hline & File & module-4/study-reports/other-toxicity-studies/immunotoxicity/study-report-2-data.pdf \\
\hline & Comment & \\
\hline & Number & 4.2 .5 .2 .5 \\
\hline & Title & Study Report 3 \\
\hline 304 & Element & m4-2-5-2-immunotoxicity \\
\hline & File & module-4/study-reports/other-toxicity-studies/immunotoxicity/study-report-3.pdf \\
\hline & Comment & \\
\hline & Number & 4.2 .5 .2 .6 \\
\hline & Title & Study Report 3 Data \\
\hline 305 & Element & m4-2-5-2-immunotoxicity \\
\hline & File & module-4/study-reports/other-toxicity-studies/immunotoxicity/study-report-3-data.pdf \\
\hline & Comment & \\
\hline
\end{tabular}




\begin{tabular}{|c|c|c|}
\hline & Number & 4.2 .5 .3 \\
\hline & Title & Mechanistic studies (if not included elsewhere) \\
\hline 306 & Element & m4-2-5-3-mechanistic-studies \\
\hline & Directory & module-4/study-reports/other-toxicity-studies/mechanistic-studies \\
\hline & Comment & \\
\hline & Number & 4.2 .5 .3 .1 \\
\hline & Title & Study Report 1 \\
\hline 307 & Element & m4-2-5-3-mechanistic-studies \\
\hline & File & module-4/study-reports/other-toxicity-studies/mechanistic-studies/study-report-1.pdf \\
\hline & Comment & \\
\hline & Number & 4.2 .5 .3 .2 \\
\hline & Title & Study Report 1 Data \\
\hline 308 & Element & m4-2-5-3-mechanistic-studies \\
\hline & File & module-4/study-reports/other-toxicity-studies/mechanistic-studies/study-report-1-data.pdf \\
\hline & Comment & \\
\hline & Number & 4.2 .5 .3 .3 \\
\hline & Title & Study Report 2 \\
\hline 309 & Element & m4-2-5-3-mechanistic-studies \\
\hline & File & module-4/study-reports/other-toxicity-studies/mechanistic-studies/study-report-2.pdf \\
\hline & Comment & \\
\hline & Number & 4.2 .5 .3 .4 \\
\hline & Title & Study Report 2 Data \\
\hline 310 & Element & m4-2-5-3-mechanistic-studies \\
\hline & File & module-4/study-reports/other-toxicity-studies/mechanistic-studies/study-report-2-data.pdf \\
\hline & Comment & \\
\hline 311 & Number & 4.2 .5 .3 .5 \\
\hline & Title & Study Report 3 \\
\hline & Element & m4-2-5-3-mechanistic-studies \\
\hline & File & module-4/study-reports/other-toxicity-studies/mechanistic-studies/study-report-3.pdf \\
\hline
\end{tabular}




\begin{tabular}{|c|c|c|}
\hline & Comment & \\
\hline & Number & 4.2 .5 .3 .6 \\
\hline & Title & Study Report 3 Data \\
\hline 312 & Element & m4-2-5-3-mechanistic-studies \\
\hline & File & module-4/study-reports/other-toxicity-studies/mechanistic-studies/study-report-3-data.pdf \\
\hline & Comment & \\
\hline & Number & 4.2 .5 .4 \\
\hline & Title & Dependence \\
\hline 313 & Element & m4-2-5-4-dependence \\
\hline & Directory & module-4/study-reports/other-toxicity-studies/dependence \\
\hline & Comment & \\
\hline & Number & 4.2 .5 .4 .1 \\
\hline & Title & Study Report 1 \\
\hline 314 & Element & m4-2-5-4-dependence \\
\hline & File & module-4/study-reports/other-toxicity-studies/dependence/study-report-1.pdf \\
\hline & Comment & \\
\hline & Number & 4.2 .5 .4 .2 \\
\hline & Title & Study Report 1 Data \\
\hline 315 & Element & m4-2-5-4-dependence \\
\hline & File & module-4/study-reports/other-toxicity-studies/dependence/study-report-1-data.pdf \\
\hline & Comment & \\
\hline & Number & 4.2 .5 .4 .3 \\
\hline & Title & Study Report 2 \\
\hline 316 & Element & m4-2-5-4-dependence \\
\hline & File & module-4/study-reports/other-toxicity-studies/dependence/study-report-2.pdf \\
\hline & Comment & \\
\hline 317 & Number & 4.2 .5 .4 .4 \\
\hline & Title & Study Report 2 Data \\
\hline & Element & m4-2-5-4-dependence \\
\hline
\end{tabular}




\begin{tabular}{|c|c|c|}
\hline & File & module-4/study-reports/other-toxicity-studies/dependence/study-report-2-data.pdf \\
\hline & Comment & \\
\hline & Number & 4.2 .5 .4 .5 \\
\hline & Title & Study Report 3 \\
\hline 318 & Element & m4-2-5-4-dependence \\
\hline & File & module-4/study-reports/other-toxicity-studies/dependence/study-report-3.pdf \\
\hline & Comment & \\
\hline & Number & 4.2 .5 .4 .6 \\
\hline & Title & Study Report 3 Data \\
\hline 319 & Element & m4-2-5-4-dependence \\
\hline & File & module-4/study-reports/other-toxicity-studies/dependence/study-report-3-data.pdf \\
\hline & Comment & \\
\hline & Number & 4.2 .5 .5 \\
\hline & Title & Metabolites \\
\hline 320 & Element & m4-2-5-5-metabolites \\
\hline & Directory & module-4/study-reports/other-toxicity-studies/metabolites \\
\hline & Comment & \\
\hline & Number & 4.2.5.5.1 \\
\hline & Title & Study Report 1 \\
\hline 321 & Element & m4-2-5-5-metabolites \\
\hline & File & module-4/study-reports/other-toxicity-studies/metabolites/study-report-1.pdf \\
\hline & Comment & \\
\hline & Number & 4.2 .5 .5 .2 \\
\hline & Title & Study Report 1 Data \\
\hline 322 & Element & m4-2-5-5-metabolites \\
\hline & File & module-4/study-reports/other-toxicity-studies/metabolites/study-report-1-data.pdf \\
\hline & Comment & \\
\hline 323 & Number & 4.2.5.5.3 \\
\hline & Title & Study Report 2 \\
\hline
\end{tabular}




\begin{tabular}{|c|c|c|}
\hline & \begin{tabular}{|l|} 
Element \\
File
\end{tabular} & \begin{tabular}{|l|} 
m4-2-5-5-metabolites \\
module-4/study-reports/other-toxicity-studies/metabolites/study-report-2.pdf
\end{tabular} \\
\hline & Comment & \\
\hline & Number & 4.2.5.5.4 \\
\hline & Title & Study Report 2 Data \\
\hline 324 & Element & m4-2-5-5-metabolites \\
\hline & File & module-4/study-reports/other-toxicity-studies/metabolites/study-report-2-data.pdf \\
\hline & Comment & \\
\hline & Number & 4.2 .5 .5 .5 \\
\hline & Title & Study Report 3 \\
\hline 325 & Element & m4-2-5-5-metabolites \\
\hline & File & module-4/study-reports/other-toxicity-studies/metabolites/study-report-3.pdf \\
\hline & Comment & \\
\hline & Number & 4.2 .5 .5 .6 \\
\hline & Title & Study Report 3 Data \\
\hline 326 & Element & m4-2-5-5-metabolites \\
\hline & File & module-4/study-reports/other-toxicity-studies/metabolites/study-report-3-data.pdf \\
\hline & Comment & \\
\hline & Number & 4.2 .5 .6 \\
\hline & Title & Impurities \\
\hline $327 \mid$ & Element & m4-2-5-6-impurities \\
\hline & Directory & module-4/study-reports/other-toxicity-studies/impurities \\
\hline & Comment & \\
\hline & Number & 4.2.5.6.1 \\
\hline & Title & Study Report 1 \\
\hline 328 & Element & m4-2-5-6-impurities \\
\hline & File & module-4/study-reports/other-toxicity-studies/impurities/study-report-1.pdf \\
\hline & Comment & \\
\hline 329 & Number & 4.2.5.6.2 \\
\hline
\end{tabular}




\begin{tabular}{|c|c|c|}
\hline & Title & Study Report 1 Data \\
\hline & Element & m4-2-5-6-impurities \\
\hline & File & module-4/study-reports/other-toxicity-studies/impurities/study-report-1-data.pdf \\
\hline & Comment & \\
\hline & Number & 4.2 .5 .6 .3 \\
\hline & Title & Study Report 2 \\
\hline 330 & Element & m4-2-5-6-impurities \\
\hline & File & module-4/study-reports/other-toxicity-studies/impurities/study-report-2.pdf \\
\hline & Comment & \\
\hline & Number & 4.2 .5 .6 .4 \\
\hline & Title & Study Report 2 Data \\
\hline 331 & Element & m4-2-5-6-impurities \\
\hline & File & module-4/study-reports/other-toxicity-studies/impurities/study-report-2-data.pdf \\
\hline & Comment & \\
\hline & Number & 4.2 .5 .6 .5 \\
\hline & Title & Study Report 3 \\
\hline 332 & Element & m4-2-5-6-impurities \\
\hline & File & module-4/study-reports/other-toxicity-studies/impurities/study-report-3.pdf \\
\hline & Comment & \\
\hline & Number & 4.2 .5 .6 .6 \\
\hline & Title & Study Report 3 Data \\
\hline 333 & Element & m4-2-5-6-impurities \\
\hline & File & module-4/study-reports/other-toxicity-studies/impurities/study-report-3-data.pdf \\
\hline & Comment & \\
\hline & Number & 4.2 .5 .7 \\
\hline & Title & Other \\
\hline 334 & Element & m4-2-5-7-other \\
\hline & \begin{tabular}{|l|} 
Directory \\
\end{tabular} & module-4/study-reports/other-toxicity-studies/other \\
\hline & Comment & \\
\hline
\end{tabular}




\begin{tabular}{|c|c|c|}
\hline & Number & 4.2 .5 .7 .1 \\
\hline & Title & Study Report 1 \\
\hline $335[1$ & Element & m4-2-5-7-other \\
\hline & File & module-4/study-reports/other-toxicity-studies/other/study-report-1.pdf \\
\hline & Comment & \\
\hline & Number & $4 \cdot 2 \cdot 5 \cdot 7.2$ \\
\hline & Title & Study Report 1 Data \\
\hline 336 & Element & m4-2-5-7-other \\
\hline & File & module-4/study-reports/other-toxicity-studies/other/study-report-1-data.pdf \\
\hline & Comment & \\
\hline & Number & $4.2 \cdot 5 \cdot 7.3$ \\
\hline & Title & Study Report 2 \\
\hline 3371 & Element & m4-2-5-7-other \\
\hline & File & module-4/study-reports/other-toxicity-studies/other/study-report-2.pdf \\
\hline & Comment & \\
\hline & Number & $4 \cdot 2 \cdot 5 \cdot 7.4$ \\
\hline & Title & Study Report 2 Data \\
\hline 338 & Element & m4-2-5-7-other \\
\hline & File & module-4/study-reports/other-toxicity-studies/other/study-report-2-data.pdf \\
\hline & Comment & \\
\hline & Number & $4 \cdot 2 \cdot 5 \cdot 7.5$ \\
\hline & Title & Study Report 3 \\
\hline 339 & Element & m4-2-5-7-other \\
\hline & File & module-4/study-reports/other-toxicity-studies/other/study-report-3.pdf \\
\hline & Comment & \\
\hline 340 & Number & $4 \cdot 2 \cdot 5 \cdot 7.6$ \\
\hline & Title & Study Report 3 Data \\
\hline & Element & m4-2-5-7-other \\
\hline & File & module-4/study-reports/other-toxicity-studies/other/study-report-3-data.pdf \\
\hline
\end{tabular}




\begin{tabular}{|c|c|c|}
\hline & Comment & \\
\hline & Number & 4.3 \\
\hline & Title & Literature References \\
\hline 341 & 1 Element & m4-3-literature-references \\
\hline & Directory & module-4/references \\
\hline & Comment & \\
\hline & Number & 4.3 .1 \\
\hline & Title & Reference 1 \\
\hline & Element & m4-3-literature-references \\
\hline 342 & $2 \longdiv { \text { File } }$ & module-4/references/reference-1.pdf \\
\hline & Comment & $\begin{array}{l}\text { Applicants can use an alternative approach whereby a single PDF file includes all references with bookmarks to each } \\
\text { tindividual reference. However, this option means that the whole file should be replaced if any update is made to its } \\
\text { components. }\end{array}$ \\
\hline & Number & 4.3 .2 \\
\hline & Title & Reference 2 \\
\hline 343 & $3 \longdiv { \text { Element } }$ & m4-3-literature-references \\
\hline & File & module-4/references/reference-2.pdf \\
\hline & Comment & \\
\hline & Number & 4.3 .3 \\
\hline & Title & Reference 3 \\
\hline 344 & 4 Element & m4-3-literature-references \\
\hline & File & module-4/references/reference-3.pdf \\
\hline & Comment & \\
\hline
\end{tabular}




\begin{tabular}{|c|c|c|}
\hline & Number & 5 \\
\hline & Title & Clinical Study Reports \\
\hline 345 & Element & m5-clinical-study-reports \\
\hline & Directory & module-5 \\
\hline & Comment & \\
\hline & Number & 5.2 \\
\hline & Title & Tabular Listing of all Clinical Studies \\
\hline 346 & Element & m5-2-tabular-listing-of-all-clinical-studies \\
\hline & File & module-5/tabular-listing.pdf \\
\hline & Comment & \\
\hline & Number & 5.3 \\
\hline & Title & Clinical Study Reports \\
\hline 347 & Element & m5-3-clinical-study-reports \\
\hline & Directory & module-5/clinical-study-reports \\
\hline & Comment & \\
\hline & Number & 5.3 .1 \\
\hline & Title & Reports of Biopharmaceutic Studies \\
\hline 348 & Element & m5-3-1-reports-of-biopharmaceutic-studies \\
\hline & Directory & module-5/clinical-study-reports/biopharmaceutic-studies \\
\hline & Comment & \\
\hline & Number & 5.3.1.1 \\
\hline & Title & Bioavailability (BA) Study Reports \\
\hline 349 & Element & m5-3-1-1-bioavailability-study-reports \\
\hline & Directory & module-5/clinical-study-reports/biopharmaceutic-studies/bioavailability \\
\hline & Comment & \\
\hline 350 & Number & 5.3 .1 .1 .1 \\
\hline & Title & Study Report 1 \\
\hline & Element & m5-3-1-1-bioavailability-study-reports \\
\hline
\end{tabular}




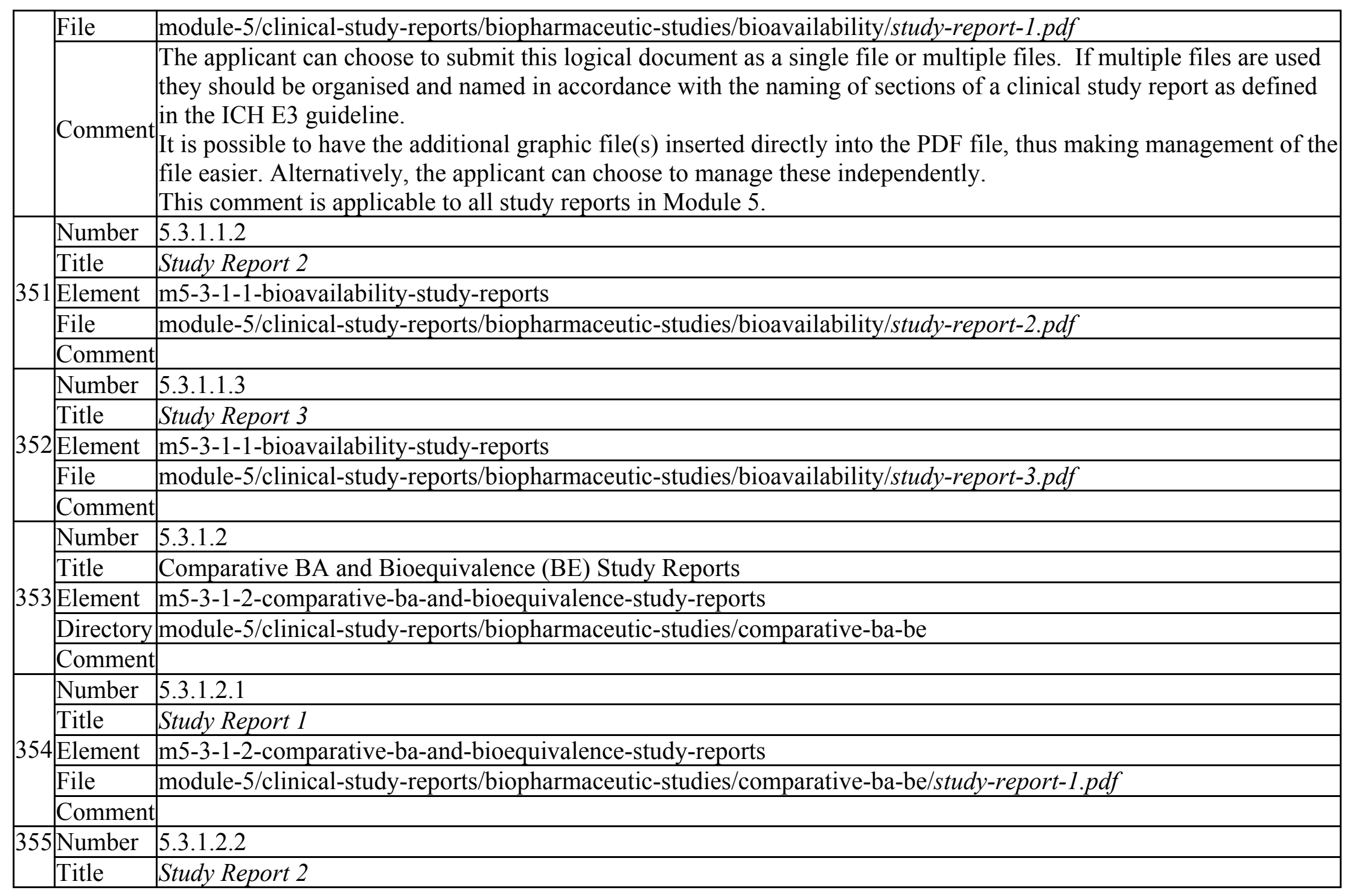




\begin{tabular}{|l|l|l|}
\hline Element & m5-3-1-2-comparative-ba-and-bioequivalence-study-reports \\
\hline File & module-5/clinical-study-reports/biopharmaceutic-studies/comparative-ba-be/study-report-2.pdf \\
\hline Comment & \\
\hline Number & 5.3 .1 .2 .3 \\
\hline 356 & Title & Study Report 3 \\
\hline Element & m5-3-1-2-comparative-ba-and-bioequivalence-study-reports \\
\hline File & module-5/clinical-study-reports/biopharmaceutic-studies/comparative-ba-be/study-report-3.pdf \\
\hline Comment & \\
\hline Number & 5.3 .1 .3 \\
\hline 357 & Title & In vitro - In vivo Correlation Study Reports \\
\hline Element & m5-3-1-3-in-vitro-in-vivo-correlation-study-reports \\
\hline Directory & module-5/clinical-study-reports/biopharmaceutic-studies/in-vitro-in-vivo \\
\hline Comment & \\
\hline Number & 5.3.1.3.1 \\
\hline Title & Study Report 1 \\
\hline 358 & Element & m5-3-1-3-in-vitro-in-vivo-correlation-study-reports \\
\hline File & module-5/clinical-study-reports/biopharmaceutic-studies/in-vitro-in-vivo/study-report-1.pdf \\
\hline Comment & \\
\hline Number & 5.3 .1 .3 .2 \\
\hline Title & Study Report 2 \\
\hline Element & m5-3-1-3-in-vitro-in-vivo-correlation-study-reports \\
\hline File & module-5/clinical-study-reports/biopharmaceutic-studies/in-vitro-in-vivo/study-report-2.pdf \\
\hline Comment & \\
\hline Number & 5.3 .1 .3 .3 \\
\hline Title & Study Report 3 \\
\hline Element & m5-3-1-3-in-vitro-in-vivo-correlation-study-reports \\
\hline File & module-5/clinical-study-reports/biopharmaceutic-studies/in-vitro-in-vivo/study-report-3.pdf \\
\hline Comment & \\
\hline 361 & Number & 5.3 .1 .4 \\
\hline
\end{tabular}

Page 4-69 


\begin{tabular}{|c|c|c|}
\hline & \begin{tabular}{|l|} 
Title \\
Element
\end{tabular} & \begin{tabular}{|l|} 
Reports of Bioanalytical and Analytical Methods for Human Studies \\
m5-3-1-4-reports-of-bioanalvtical-and-analytical-methods-for-human-studies
\end{tabular} \\
\hline & \begin{tabular}{|l|l} 
Element \\
Directory
\end{tabular} & module-5/clinical-study-reports/biopharmaceutic-studies/bioanalyt-analyt-methods \\
\hline & Comment & \\
\hline & Number & 5.3.1.4.1 \\
\hline & Title & Study Report 1 \\
\hline 362 & Element & m5-3-1-4-reports-of-bioanalytical-and-analytical-methods-for-human-studies \\
\hline & File & module-5/clinical-study-reports/biopharmaceutic-studies/bioanalyt-analyt-methods/study-report-1.pdf \\
\hline & Comment & \\
\hline & Number & 5.3.1.4.2 \\
\hline & Title & Study Report 2 \\
\hline 363 & Element & m5-3-1-4-reports-of-bioanalytical-and-analytical-methods-for-human-studies \\
\hline & File & module-5/clinical-study-reports/biopharmaceutic-studies/bioanalyt-analyt-methods/study-report-2.pdf \\
\hline & Comment & \\
\hline & Number & 5.3.1.4.3 \\
\hline & Title & Study Report 3 \\
\hline 364 & Element & m5-3-1-4-reports-of-bioanalytical-and-analytical-methods-for-human-studies \\
\hline & File & module-5/clinical-study-reports/biopharmaceutic-studies/bioanalyt-analyt-methods/study-report-3.pdf \\
\hline & Comment & \\
\hline & Number & 5.3 .2 \\
\hline & Title & Reports of Studies Pertinent to Pharamacokinetics using Human Biomaterials \\
\hline 365 & Element & m5-3-2-reports-of-studies-pertinent-to-pharmacokinetics-using-human-biomaterials \\
\hline & Directory & module-5/clinical-study-reports/pk-human-biomaterials \\
\hline & Comment & \\
\hline & Number & 5.3.2.1 \\
\hline & Title & Plasma Protein Binding Study Reports \\
\hline 366 & Element & m5-3-2-1-plasma-protein-binding-study-reports \\
\hline & Director & module-5/clinical-study-reports/pk-human-biomaterials/plasma-protein-binding \\
\hline & Com & \\
\hline
\end{tabular}

Page 4-70 


\begin{tabular}{|c|c|c|}
\hline & Number & 5.3 .2 .1 .1 \\
\hline & Title & Study Report 1 \\
\hline 367 & Element & m5-3-2-1-plasma-protein-binding-study-reports \\
\hline & File & module-5/clinical-study-reports/pk-human-biomaterials/plasma-protein-binding/study-report-1.pdf \\
\hline & Comment & \\
\hline & Number & 5.3.2.1.2 \\
\hline & Title & Study Report 2 \\
\hline 368 & Element & m5-3-2-1-plasma-protein-binding-study-reports \\
\hline & File & module-5/clinical-study-reports/pk-human-biomaterials/plasma-protein-binding/study-report-2.pdf \\
\hline & Comment & \\
\hline & Number & 5.3.2.1.3 \\
\hline & Title & Study Report 3 \\
\hline 369 & Element & m5-3-2-1-plasma-protein-binding-study-reports \\
\hline & File & module-5/clinical-study-reports/pk-human-biomaterials/plasma-protein-binding/study-report-3.pdf \\
\hline & Comment & \\
\hline & Number & 5.3 .2 .2 \\
\hline & Title & Reports of Hepatic Metabolism and Drug Interaction Studies \\
\hline 370 & Element & m5-3-2-2-reports-of-hepatic-metabolism-and-drug-interaction-studies \\
\hline & Directory & module-5/clinical-study-reports/pk-human-biomaterials/hepatic-metab-interactions \\
\hline & Comment & \\
\hline & Number & 5.3.2.2.1 \\
\hline & Title & Study Report 1 \\
\hline 371 & Element & m5-3-2-2-reports-of-hepatic-metabolism-and-drug-interaction-studies \\
\hline & File & module-5/clinical-study-reports/pk-human-biomaterials/hepatic-metab-interactions/study-report-1.pdf \\
\hline & Comment & \\
\hline 372 & Number & 5.3 .2 .2 .2 \\
\hline & Title & Study Report 2 \\
\hline & Element & m5-3-2-2-reports-of-hepatic-metabolism-and-drug-interaction-studies \\
\hline & File & module-5/clinical-study-reports/pk-human-biomaterials/hepatic-metab-interactions/study-report-2.pdf \\
\hline
\end{tabular}




\begin{tabular}{|c|c|c|}
\hline & Comment & \\
\hline \multirow{5}{*}{373} & Number & 5.3.2.2.3 \\
\hline & Title & Study Report 3 \\
\hline & Element & m5-3-2-2-reports-of-hepatic-metabolism-and-drug-interaction-studies \\
\hline & File & module-5/clinical-study-reports/pk-human-biomaterials/hepatic-metab-interactions/study-report-3.pdf \\
\hline & Comment & \\
\hline \multirow{5}{*}{374} & Number & 5.3.2.3 \\
\hline & Title & Reports of Studies Using Other Human Biomaterials \\
\hline & Element & m5-3-2-3-reports-of-studies-using-other-human-biomaterials \\
\hline & Directory & module-5/clinical-study-reports/pk-human-biomaterials/other-human-biomaterials \\
\hline & Comment & \\
\hline \multirow{5}{*}{375} & Number & 5.3 .2 .3 .1 \\
\hline & Title & Study Report 1 \\
\hline & Element & m5-3-2-3-reports-of-studies-using-other-human-biomaterials \\
\hline & File & module-5/clinical-study-reports/pk-human-biomaterials/other-human-biomaterials/study-report-1.pdf \\
\hline & Comment & \\
\hline \multirow{5}{*}{376} & Number & 5.3 .2 .3 .2 \\
\hline & Title & Study Report 2 \\
\hline & Element & m5-3-2-3-reports-of-studies-using-other-human-biomaterials \\
\hline & File & module-5/clinical-study-reports/pk-human-biomaterials/other-human-biomaterials/study-report-2.pdf \\
\hline & Comment & \\
\hline \multirow{5}{*}{377} & Number & 5.3 .2 .3 .3 \\
\hline & Title & Study Report 3 \\
\hline & Element & m5-3-2-3-reports-of-studies-using-other-human-biomaterials \\
\hline & File & module-5/clinical-study-reports/pk-human-biomaterials/other-human-biomaterials/study-report-3.pdf \\
\hline & Comment & \\
\hline \multirow[t]{3}{*}{378} & Number & 5.3 .3 \\
\hline & Title & Reports of Human Pharmacokinetic (PK) Studies \\
\hline & Element & m5-3-3-reports-of-human-pharmacokinetics-pk-studies \\
\hline
\end{tabular}

Page 4-72 


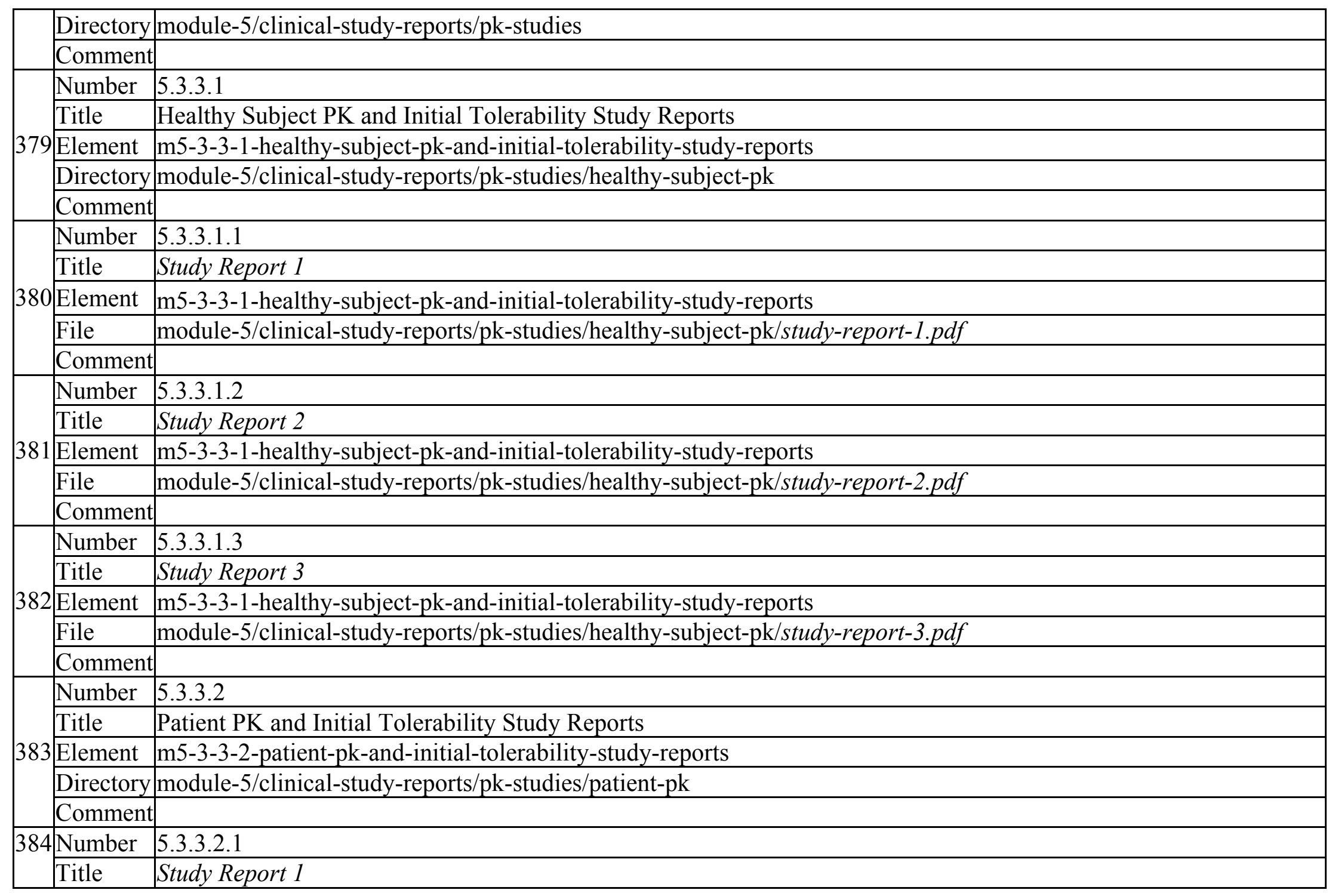




\begin{tabular}{|c|c|c|}
\hline & \begin{tabular}{|l|} 
Element \\
File
\end{tabular} & \begin{tabular}{|l} 
m5-3-3-2-patient-pk-and-initial-tolerability-study-reports \\
module-5/clinical-study-reports/pk-studies/patient-pk/studv-report-1.pdf
\end{tabular} \\
\hline & Comment & \\
\hline & Number & 5.3.3.2.2 \\
\hline & Title & Study Report 2 \\
\hline 385 & Element & m5-3-3-2-patient-pk-and-initial-tolerability-study-reports \\
\hline & File & module-5/clinical-study-reports/pk-studies/patient-pk/study-report-2.pdf \\
\hline & Comment & \\
\hline & Number & 5.3.3.2.3 \\
\hline & Title & Study Report 3 \\
\hline 386 & Element & m5-3-3-2-patient-pk-and-initial-tolerability-study-reports \\
\hline & File & module-5/clinical-study-reports/pk-studies/patient-pk/study-report-3.pdf \\
\hline & Comment & \\
\hline & Number & 5.3 .3 .3 \\
\hline & Title & Intrinsic Factor PK Study Reports \\
\hline 387 & Element & m5-3-3-3-intrinsic-factor-pk-study-reports \\
\hline & Directory & module-5/clinical-study-reports/pk-studies/intrinsic-factor-pk \\
\hline & Comment & \\
\hline & Number & 5.3.3.3.1 \\
\hline & Title & Study Report 1 \\
\hline 388 & Element & m5-3-3-3-intrinsic-factor-pk-study-reports \\
\hline & File & module-5/clinical-study-reports/pk-studies/intrinsic-factor-pk/study-report-1.pdf \\
\hline & Comment & \\
\hline & Number & 5.3.3.3.2 \\
\hline & Title & Study Report 2 \\
\hline 389 & Element & m5-3-3-3-intrinsic-factor-pk-study-reports \\
\hline & File & module-5/clinical-study-reports/pk-studies/intrinsic-factor-pk/study-report-2.pdf \\
\hline & Comment & \\
\hline 390 & Number & 5.3.3.3.3 \\
\hline
\end{tabular}

Page 4-74 


\begin{tabular}{|c|c|c|}
\hline & \begin{tabular}{|l|} 
Title \\
Element
\end{tabular} & $\begin{array}{l}\text { Study Report } 3 \\
\text { m5-3-3-3-intrinsic-factor-pk-studv-reports }\end{array}$ \\
\hline & \begin{tabular}{|l|} 
Element \\
File
\end{tabular} & module-5/clinical-studv-reports/pk-studies/intrinsic-factor-pk/studv-report-3.pdf \\
\hline & Comment & \\
\hline & Number & 5.3.3.4 \\
\hline & Title & Extrinsic Factor PK Study Reports \\
\hline 391 & Element & m5-3-3-4-extrinsic-factor-pk-study-reports \\
\hline & Directory & module-5/clinical-study-reports/pk-studies/extrinsic-factor-pk \\
\hline & Comment & \\
\hline & Number & 5.3.3.4.1 \\
\hline & Title & Study Report 1 \\
\hline 392 & Element & m5-3-3-4-extrinsic-factor-pk-study-reports \\
\hline & File & module-5/clinical-study-reports/pk-studies/extrinsic-factor-pk/study-report-1.pdf \\
\hline & Comment & \\
\hline & Number & 5.3.3.4.2 \\
\hline & Title & Study Report 2 \\
\hline 393 & Element & m5-3-3-4-extrinsic-factor-pk-study-reports \\
\hline & File & module-5/clinical-study-reports/pk-studies/extrinsic-factor-pk/study-report-2.pdf \\
\hline & Comment & \\
\hline & Number & 5.3.3.4.3 \\
\hline & Title & Study Report 3 \\
\hline 394 & Element & m5-3-3-4-extrinsic-factor-pk-study-reports \\
\hline & File & module-5/clinical-study-reports/pk-studies/extrinsic-factor-pk/study-report-3.pdf \\
\hline & Comment & \\
\hline & Number & 5.3.3.5 \\
\hline & Title & Population PK Study Reports \\
\hline 395 & Element & m5-3-3-5-population-pk-study-reports \\
\hline & Directory & module-5/clinical-study-reports/pk-studies/population-pk \\
\hline & Comment & \\
\hline
\end{tabular}

Page 4-75 


\begin{tabular}{|c|c|c|}
\hline & Number & 5.3.3.5.1 \\
\hline & Title & Study Report 1 \\
\hline 396 & Element & m5-3-3-5-population-pk-study-reports \\
\hline & File & module-5/clinical-study-reports/pk-studies/population-pk/study-report-1.pdf \\
\hline & Comment & \\
\hline & Number & 5.3 .3 .5 .2 \\
\hline & Title & Study Report 2 \\
\hline 397 & Element & m5-3-3-5-population-pk-study-reports \\
\hline & File & module-5/clinical-study-reports/pk-studies/population-pk/study-report-2.pdf \\
\hline & Comment & \\
\hline & Number & 5.3.3.5.3 \\
\hline & Title & Study Report 3 \\
\hline 398 & Element & m5-3-3-5-population-pk-study-reports \\
\hline & File & module-5/clinical-study-reports/pk-studies/population-pk/study-report-3.pdf \\
\hline & Comment & \\
\hline & Number & 5.3 .4 \\
\hline & Title & Reports of Human Pharmacodynamic (PD) Studies \\
\hline 399 & Element & m5-3-4-reports-of-human-pharmacodynamics-pd-studies \\
\hline & Directory & module-5/clinical-study-reports/human-pd-studies \\
\hline & Comment & \\
\hline & Number & 5.3 .4 .1 \\
\hline & Title & Healthy Subject PD and PK/PD Study Reports \\
\hline 400 & Element & m5-3-4-1-healthy-subject-pd-and-pk-pd-study-reports \\
\hline & Directory & module-5/clinical-study-reports/human-pd-studies/healthy-subject-pd \\
\hline & Comment & \\
\hline 401 & Number & 5.3 .4 .1 .1 \\
\hline & Title & Study Report 1 \\
\hline & Element & m5-3-4-1-healthy-subject-pd-and-pk-pd-study-reports \\
\hline & File & module-5/clinical-study-reports/human-pd-studies/healthy-subject-pd/study-report-1.pdf \\
\hline
\end{tabular}




\begin{tabular}{|c|c|c|}
\hline & Comment & \\
\hline & Number & 5.3.4.1.2 \\
\hline & Title & Study Report 2 \\
\hline 402 & 2 Element & m5-3-4-1-healthy-subject-pd-and-pk-pd-study-reports \\
\hline & File & module-5/clinical-study-reports/human-pd-studies/healthy-subject-pd/study-report-2.pdf \\
\hline & Comment & \\
\hline & Number & 5.3.4.1.3 \\
\hline & Title & Study Report 3 \\
\hline 403 & 3 Element & m5-3-4-1-healthy-subject-pd-and-pk-pd-study-reports \\
\hline & File & module-5/clinical-study-reports/human-pd-studies/healthy-subject-pd/study-report-3.pdf \\
\hline & Comment & \\
\hline & Number & 5.3.4.2 \\
\hline & Title & Patient PD and PK/PD Study Reports \\
\hline 404 & 4 Element & m5-3-4-2-patient-pd-and-pk-pd-study-reports \\
\hline & Directory & module-5/clinical-study-reports/human-pd-studies/patient-pd \\
\hline & Comment & \\
\hline & Number & 5.3.4.2.1 \\
\hline & Title & Study Report 1 \\
\hline 405 & 5 Element & m5-3-4-2-patient-pd-and-pk-pd-study-reports \\
\hline & File & module-5/clinical-study-reports/human-pd-studies/patient-pd/study-report-1.pdf \\
\hline & Comment & \\
\hline & Number & 5.3.4.2.2 \\
\hline & Title & Study Report 2 \\
\hline 406 & 6 Element & m5-3-4-2-patient-pd-and-pk-pd-study-reports \\
\hline & File & module-5/clinical-study-reports/human-pd-studies/patient-pd/study-report-2.pdf \\
\hline & Comment & \\
\hline 407 & 7 Number & 5.3.4.2.3 \\
\hline & Title & Study Report 3 \\
\hline & Element & m5-3-4-2-patient-pd-and-pk-pd-study-reports \\
\hline
\end{tabular}




\begin{tabular}{|c|c|c|}
\hline & File & module-5/clinical-study-reports/human-pd-studies/patient-pd/study-report-3.pdf \\
\hline & Comment & \\
\hline & Number & 5.3 .5 \\
\hline & Title & Reports of Efficacy and Safety Studies \\
\hline 408 & \begin{tabular}{|l|l} 
Element & 1 \\
\end{tabular} & m5-3-5-reports-of-efficacy-and-safety-studies \\
\hline & Directory 1 & module-5/clinical-study-reports/efficacy-safety \\
\hline & Comment & \\
\hline & Number & 5.3 .5 \\
\hline & Title & Reports of Efficacy and Safety Studies - Indication Name \\
\hline & \begin{tabular}{|l|l} 
Element & 1
\end{tabular} & m5-3-5-reports-of-efficacy-and-safety-studies \\
\hline 409 & Directory 1 & module-5/clinical-study-reports/efficacy-safety/indication-1 \\
\hline & Comment & $\begin{array}{l}\text { The folder name should always include the indication being claimed (abbreviated if appropriate) eg. 'asthma'. Where } \\
\text { there is more than one indication (eg asthma \& migraine) then the first indication has a folder 'asthma' and the } \\
\text { second 'migraine'. }\end{array}$ \\
\hline & Number & 5.3 .5 .1 \\
\hline & Title & Study Reports of Controlled Clinical Studies Pertinent to the Claimed Indication \\
\hline 410 & \begin{tabular}{|l|l} 
Element & 1 \\
\end{tabular} & m5-3-5-1-study-reports-of-controlled-clinical-studies-pertinent-to-the-claimed-indication \\
\hline & Directory 1 & module-5/clinical-study-reports/efficacy-safety/indication-1/controlled-studies \\
\hline & Comment & \\
\hline & Number & 5.3 .5 .1 .1 \\
\hline & Title & Study Report 1 \\
\hline 411 & \begin{tabular}{|l|l} 
Element & 1 \\
\end{tabular} & m5-3-5-1-study-reports-of-controlled-clinical-studies-pertinent-to-the-claimed-indication \\
\hline & File & module-5/clinical-study-reports/efficacy-safety/indication-1/controlled-studies/study-report-1.pdf \\
\hline & Comment & \\
\hline & Number & 5.3 .5 .1 .2 \\
\hline & Title & Study Report 2 \\
\hline 412 & \begin{tabular}{|l|l} 
Element & 1 \\
\end{tabular} & m5-3-5-1-study-reports-of-controlled-clinical-studies-pertinent-to-the-claimed-indication \\
\hline & File & module-5/clinical-study-reports/efficacy-safety/indication-1/controlled-studies/study-report-2.pdf \\
\hline & Comment & \\
\hline
\end{tabular}




\begin{tabular}{|c|c|c|}
\hline \multirow{4}{*}{413} & \begin{tabular}{|l|} 
Number \\
Title
\end{tabular} & \begin{tabular}{|l|} 
5.3.5.1.3 \\
Studv Report 3 \\
\end{tabular} \\
\hline & Element & m5-3-5-1-study-reports-of-controlled-clinical-studies-pertinent-to-the-claimed-indication \\
\hline & File & module-5/clinical-study-reports/efficacy-safety/indication-1/controlled-studies/study-report-3.pdf \\
\hline & Comment & \\
\hline \multirow{5}{*}{414} & Number & 5.3 .5 .2 \\
\hline & Title & Study Reports of Uncontrolled Clinical Studies \\
\hline & Element & m5-3-5-2-study-reports-of-uncontrolled-clinical-studies \\
\hline & Directory & module-5/clinical-study-reports/efficacy-safety/indication-1/uncontrolled-studies \\
\hline & Comment & \\
\hline \multirow{5}{*}{415} & Number & 5.3.5.2.1 \\
\hline & Title & Study Report 1 \\
\hline & Element & m5-3-5-2-study-reports-of-uncontrolled-clinical-studies \\
\hline & File & module-5/clinical-study-reports/efficacy-safety/indication-1/uncontrolled-studies/study-report-1.pdf \\
\hline & Comment & \\
\hline \multirow{5}{*}{416} & Number & 5.3.5.2.2 \\
\hline & Title & Study Report 2 \\
\hline & Element & m5-3-5-2-study-reports-of-uncontrolled-clinical-studies \\
\hline & File & module-5/clinical-study-reports/efficacy-safety/indication-1/uncontrolled-studies/study-report-2.pdf \\
\hline & Comment & \\
\hline \multirow{5}{*}{417} & Number & 5.3.5.2.3 \\
\hline & Title & Study Report 3 \\
\hline & Element & m5-3-5-2-study-reports-of-uncontrolled-clinical-studies \\
\hline & File & module-5/clinical-study-reports/efficacy-safety/indication-1/uncontrolled-studies/study-report-3.pdf \\
\hline & Comment & \\
\hline \multirow[t]{4}{*}{418} & Number & 5.3 .5 .3 \\
\hline & Title & Reports of Analyses of Data from More than One Study \\
\hline & Element & m5-3-5-3-reports-of-analyses-of-data-from-more-than-one-study \\
\hline & Direc & nodule-5/clinical-study-reports/efficacy-safety/indication-1/multistudy-analyses \\
\hline
\end{tabular}

Page 4-79 


\begin{tabular}{|c|c|c|}
\hline & Comment & \\
\hline \multirow{5}{*}{419} & Number & 5.3 .5 .3 .1 \\
\hline & Title & Study Report 1 \\
\hline & Element & m5-3-5-3-reports-of-analyses-of-data-from-more-than-one-study \\
\hline & File & module-5/clinical-study-reports/efficacy-safety/indication-1/multistudy-analyses/study-report-1.pdf \\
\hline & Comment & \\
\hline \multirow{5}{*}{420} & Number & 5.3 .5 .3 .2 \\
\hline & Title & Study Report 2 \\
\hline & Element & m5-3-5-3-reports-of-analyses-of-data-from-more-than-one-study \\
\hline & File & module-5/clinical-study-reports/efficacy-safety/indication-1/multistudy-analyses/study-report-2.pdf \\
\hline & Comment & \\
\hline \multirow{5}{*}{421} & Number & 5.3 .5 .3 .3 \\
\hline & Title & Study Report 3 \\
\hline & Element & m5-3-5-3-reports-of-analyses-of-data-from-more-than-one-study \\
\hline & File & module-5/clinical-study-reports/efficacy-safety/indication-1/multistudy-analyses/study-report-3.pdf \\
\hline & Comment & \\
\hline \multirow{5}{*}{422} & Number & 5.3.5.4 \\
\hline & Title & Other Study Reports \\
\hline & Element & m5-3-5-4-other-study-reports \\
\hline & Directory & module-5/clinical-study-reports/efficacy-safety/indication-1/other-studies \\
\hline & Comment & \\
\hline \multirow{5}{*}{423} & Number & 5.3.5.4.1 \\
\hline & Title & Study Report 1 \\
\hline & Element & m5-3-5-4-other-study-reports \\
\hline & File & module-5/clinical-study-reports/efficacy-safety/indication-1/other-studies/study-report-1.pdf \\
\hline & Comment & \\
\hline \multirow[t]{3}{*}{424} & Number & 5.3 .5 .4 .2 \\
\hline & Title & Study Report 2 \\
\hline & Element & m5-3-5-4-other-study-reports \\
\hline
\end{tabular}




\begin{tabular}{|c|c|c|}
\hline & File & module-5/clinical-study-reports/efficacy-safety/indication-1/other-studies/study-report-2.pdf \\
\hline & Comment & \\
\hline \multirow{5}{*}{425} & Number & 5.3.5.4.3 \\
\hline & Title & Study Report 3 \\
\hline & Element & m5-3-5-4-other-study-reports \\
\hline & File & module-5/clinical-study-reports/efficacy-safety/indication-1/other-studies/study-report-3.pdf \\
\hline & Comment & \\
\hline \multirow{5}{*}{426} & Number & 5.3 .6 \\
\hline & Title & Reports of Postmarketing Experience \\
\hline & Element & m5-3-6-reports-of-postmarketing-experience \\
\hline & Directory & module-5/clinical-study-reports/postmarketing-experience \\
\hline & Comment & \\
\hline \multirow{5}{*}{427} & Number & 5.3 .7 \\
\hline & Title & Case Report Forms and Individual Patient Listings \\
\hline & Element & m5-3-7-case-report-forms-and-individual-patient-listings \\
\hline & Directory & module-5/clinical-study-reports/crfs-patient-listings \\
\hline & Comment & \\
\hline \multirow{5}{*}{428} & Number & 5.3.7.1 \\
\hline & Title & Study 1 \\
\hline & Element & m5-3-7-case-report-forms-and-individual-patient-listings \\
\hline & Directory & module-5/clinical-study-reports/crfs-patient-listings/study-1 \\
\hline & Comment & \\
\hline \multirow{5}{*}{429} & Number & 5.3.7.1.1 \\
\hline & Title & Document/Dataset 1 \\
\hline & Element & m5-3-7-case-report-forms-and-individual-patient-listings \\
\hline & File & module-5/clinical-study-reports/crfs-patient-listings/study-1/filename-1.txt \\
\hline & Comment & $\begin{array}{l}\text { The filename and extension should include the description of the file and appropriate file extension according to } \\
\text { Appendix 2. Reference should be made to regional guidance for the acceptability of submission of datasets }\end{array}$ \\
\hline $430 \mid$ & Number & 5.3.7.1.2 \\
\hline
\end{tabular}

Page 4-81 


\begin{tabular}{|c|c|c|}
\hline & Title & Document/Dataset 2 \\
\hline & Element ${ }_{1}$ & m5-3-7-case-report-forms-and-individual-patient-listings \\
\hline & File & module-5/clinical-study-reports/crfs-patient-listings/study-1/filename-2.txt \\
\hline & Comment & \\
\hline & Number & 5.3.7.1.3 \\
\hline & Title & Document/Dataset 3 \\
\hline 431 & Element & m5-3-7-case-report-forms-and-individual-patient-listings \\
\hline & File & module-5/clinical-study-reports/crfs-patient-listings/study-1/filename-3.txt \\
\hline & Comment & \\
\hline & Number & 5.3 .7 .2 \\
\hline & Title & Study 2 \\
\hline 432 & Element 1 & m5-3-7-case-report-forms-and-individual-patient-listings \\
\hline & Directory 1 & module-5/clinical-study-reports/crfs-patient-listings/study-2 \\
\hline & Comment & define element \\
\hline & Number & 5.3.7.2.1 \\
\hline & Title & Document/Dataset 1 \\
\hline 433 & Element 1 & m5-3-7-case-report-forms-and-individual-patient-listings \\
\hline & File & module-5/clinical-study-reports/crfs-patient-listings/study-2/filename-1.txt \\
\hline & Comment & \\
\hline & Number & 5.3.7.2.2 \\
\hline & Title & Document/Dataset 2 \\
\hline 434 & Element & m5-3-7-case-report-forms-and-individual-patient-listings \\
\hline & File & module-5/clinical-study-reports/crfs-patient-listings/study-2/filename-2.txt \\
\hline & Comment & \\
\hline & Number & 5.3.7.2.3 \\
\hline & Title & Document/Dataset 3 \\
\hline 435 & Element & m5-3-7-case-report-forms-and-individual-patient-listings \\
\hline & File & module-5/clinical-study-reports/crfs-patient-listings/study-2/filename-3.txt \\
\hline & Comment & \\
\hline
\end{tabular}

Page 4-82 


\begin{tabular}{|c|c|c|}
\hline & Title & Study 3 \\
\hline 436 & 6 Element & m5-3-7-case-report-forms-and-individual-patient-listings \\
\hline & Directory & module-5/clinical-study-reports/crfs-patient-listings/study-3 \\
\hline & Comment & define element \\
\hline & Number & 5.3 .7 .3 .1 \\
\hline & Title & Document/Dataset 1 \\
\hline 437 & 7 Element & m5-3-7-case-report-forms-and-individual-patient-listings \\
\hline & File & module-5/clinical-study-reports/crfs-patient-listings/study-3/filename-1.txt \\
\hline & Comment & \\
\hline & Number & 5.3.7.3.2 \\
\hline & Title & Document/Dataset 2 \\
\hline 438 & 8 Element & m5-3-7-case-report-forms-and-individual-patient-listings \\
\hline & File & module-5/clinical-study-reports/crfs-patient-listings/study-3/filename-2.txt \\
\hline & Comment & \\
\hline & Number & 5.3 .7 .3 .3 \\
\hline & Title & Document/Dataset 3 \\
\hline 439 & 9 Element & m5-3-7-case-report-forms-and-individual-patient-listings \\
\hline & File & module-5/clinical-study-reports/crfs-patient-listings/study-3/filename-3.txt \\
\hline & Comment & \\
\hline & Number & 5.4 \\
\hline & Title & Literature References \\
\hline 440 & 0 Element & m5-4-literature-references \\
\hline & Directory & module-5/references \\
\hline & Comment & \\
\hline 441 & 1 Number & 5.4 .1 \\
\hline & Title & Reference 1 \\
\hline & Element & m5-4-literature-references \\
\hline & File & module-5/references/reference-1.pdf \\
\hline
\end{tabular}




\begin{tabular}{|c|c|c|}
\hline & Comment & $\begin{array}{l}\text { An applicant can use an alternative approach whereby a single PDF file includes all references with bookmarks to } \\
\text { each individual reference. However, this option would mean that the whole file should be replaced if any update is } \\
\text { made to its components. }\end{array}$ \\
\hline \multirow{5}{*}{442} & Number & 5.4 .2 \\
\hline & Title & Reference 2 \\
\hline & Element & m5-4-literature-references \\
\hline & File & module-5/references/reference- $2 . p d f$ \\
\hline & Comment & \\
\hline \multirow{5}{*}{443} & Number & 5.4 .3 \\
\hline & Title & Reference 3 \\
\hline & Element & m5-4-literature-references \\
\hline & File & module-5/references/reference-3.pdf \\
\hline & Comment & \\
\hline
\end{tabular}




\begin{tabular}{|c|c|c|}
\hline & Number & \\
\hline & Title & \\
\hline 444[ & Element & \\
\hline & Directory & util \\
\hline & Commen & tutilities \\
\hline & Number & \\
\hline & Title & \\
\hline $445[\mathrm{I}$ & Element & \\
\hline & Directory & util/dtd \\
\hline & Commen & tDTDs \\
\hline & Number & \\
\hline & Title & \\
\hline $446[1$ & Element & \\
\hline & File & util/dtd/ich-ectd-1-0.dtd \\
\hline & Commen & DTD for the instance - the version used to create the eCTD submission must be included \\
\hline & Number & \\
\hline & Title & \\
\hline 447 & Element & \\
\hline & File & util/dtd/eu-regional-1-0.dtd \\
\hline & Commen & DTD for the EU specific documentation \\
\hline & Number & \\
\hline & Title & \\
\hline $448 \mathrm{I}$ & Element & \\
\hline & File & util/dtd/jp-regional-1-0.dtd \\
\hline & Commen & DTD for the Japan specific documentation \\
\hline 449 & Number & \\
\hline & Title & \\
\hline & Element & \\
\hline
\end{tabular}




\begin{tabular}{|c|c|c|}
\hline & File & util/dtd/us-regional-1-0.dtd \\
\hline & Comment & DTD for the US specific documentation \\
\hline & Number & \\
\hline & Title & \\
\hline 450 & Element & \\
\hline & File & util/dtd/xx-regional-1-0.dtd \\
\hline & Comment & DTD for the $\mathrm{xx}$ specific documentation, where $\mathrm{xx}$ is a two character country code from ISO-3166-1 \\
\hline & Number & \\
\hline & Title & \\
\hline 451 & Element & \\
\hline & Directory & util/style \\
\hline & Comment & Directory for style sheets - default (ICH) and applicant specific stylesheets \\
\hline & Number & \\
\hline & Title & \\
\hline 452 & Element & \\
\hline & File & util/style/ectd-1-0.xsl \\
\hline & Comment & $\begin{array}{l}\text { The specific version of the eCTD stylesheet used by the applicant as a reference during the creation of the } \\
\text { submission should be included }\end{array}$ \\
\hline
\end{tabular}




\section{Appendix 5 Region Specific Information Including Transmission and Receipt}

\section{Introduction}

This section describes region specific information for content that is not explicitly included in the Common Technical Document and logistical details appropriate for the transmission and receipt of submissions using the electronic Common Technical Document.

\section{Region specific information: Module 1}

This module contains administrative information that is unique for each region. There will be local requirements for both the content and electronic component of module 1 . The eCTD backbone was developed to allow the transfer of this regional information to be included in a regulatory dossier.

Regional guidance will provide the specific instructions on how to provide the administrative forms and detailed prescribing information. Please refer to this information and appendix 6 when preparing module 1. Module 1 includes all administrative documents (e.g., forms and certifications) and labeling, including the documents described in regional guidance.

Not all regionally specific documents are included in module 1 . Technical reports required for a specific region should be placed in modules 2 to 5 . These reports should be included in the module most appropriate for the content of the information provided.

Each region provides specific guidance on the format and content of the regional requirements of each module. The Table 5-1 provides contact information for each region.

Table 5-1

\begin{tabular}{|c|c|c|}
\hline Region & Internet Address & Electronic Mail Contact \\
\hline European Union & http://www.emea.eu.int & esubmission@emea.eu.int \\
\hline $\begin{array}{l}\text { Food And Drug } \\
\text { Administration, USA }\end{array}$ & $\begin{array}{l}\text { http://www.fda.gov/cber } \\
\text { http://www.fda.gov/cder }\end{array}$ & $\begin{array}{l}\text { Esubprep@cber.fda.gov } \\
\text { esub@cder.fda.gov }\end{array}$ \\
\hline $\begin{array}{l}\text { Ministry of Health, Labour } \\
\text { and Welfare, Japan }\end{array}$ & $\begin{array}{l}\text { http://www.mhlw.go.jp } \\
\text { http://www.nihs.go.jp }\end{array}$ & e-submission@nihs.go.jp \\
\hline Health Canada & $\begin{array}{l}\text { http://www.hc- } \\
\text { sc.gc.ca/hpb- } \\
\text { dgps/therapeut }\end{array}$ & Bob_kapitany@hc-sc.gc.ca \\
\hline
\end{tabular}




\section{Submission Addresses}

Submissions should be sent directly to the appropriate regulatory authority. Information needed to send physical media to each regulatory authority is found at the reference location in Table 5-2.

Table 5-2

\begin{tabular}{|l|l|}
\hline \multicolumn{1}{|c|}{ Regulatory Authority } & \multicolumn{1}{c|}{ Reference location } \\
\hline $\begin{array}{l}\text { EMEA, European Union } \\
\text { Or national agencies }\end{array}$ & $\underline{\text { http://www.eudra.org/ }}$ \\
\hline $\begin{array}{l}\text { Ministry of Health, Labour and Welfare, } \\
\text { Japan }\end{array}$ & $\underline{\underline{h t t p} / / / \text { heads.medagencies.org }}$ \\
\hline $\begin{array}{l}\text { Food and Drug Admintw.go.jp } \\
\text { States of America }\end{array}$ & $\underline{\underline{\text { http://www.nihs.go.jp }}}$ \\
\hline $\begin{array}{l}\text { Health Canada, Health Protection Branch, } \\
\text { Canada }\end{array}$ & $\underline{\text { http://www.hc-sc.gc.ca/hpb-dgps/therapeut }}$ \\
\hline
\end{tabular}

\section{Media}

Regulatory authorities are prepared to accept electronic submissions provided on the media listed in Table 5-3. To optimize processing efficiency, we recommend choosing media with a capacity most appropriate to the size of the submission. Whenever possible, applicants should choose media capable of holding the submission on the fewest number of units. For example for a submission that has a size of 50 Megabytes, use 1 CD-ROM instead of 50 floppy disks.

Table 5-3

\begin{tabular}{|l|l|l|}
\hline \multicolumn{2}{|c|}{ Recommendations for Media } & Regulatory \\
Authority \\
\hline $\begin{array}{c}\text { Example Size of } \\
\text { Submission }\end{array}$ & Media and format & EU \\
\hline Less than 1.4 MB & 3.5 inch DOS Formatted Floppy Disks & USA \\
\hline Less than 10 MB & 3.5 inch DOS Formatted Floppy Disks & EU, Japan \\
\hline Less than 650 MB & CD-ROM ISO 9660 - Joliet & $\begin{array}{l}\text { Japan, USA, } \\
\text { Canada }\end{array}$ \\
\hline Less than 7 GB & CD-ROM ISO 9660 - Joliet & USA \\
\hline Greater than 7 GB & $\begin{array}{l}\text { Digital Tape - Compaq DLT 20/40 and 10/20 GB } \\
\text { format using NT server 4.0 with NT backup or } \\
\text { BackupExec }\end{array}$ & EU, Canada \\
\hline More than 650 MB & DVD & \\
\hline
\end{tabular}

\section{Cover letter}

Applicants should provide a cover letter as a PDF file (cover.pdf). A paper cover letter should also be included with non-electronic portions of the submission (such as forms with signatures or seals, and certifications). The cover letter should include: 
- A description of the submission including appropriate regulatory information.

- A listing of the sections of the submission filed as paper, electronic, or both paper and electronic.

- A description of the electronic submission including type and number of electronic media, approximate size of the submission, and, if appropriate, format used for DLT tapes.

- A statement that the submission is virus free with a description of the software used to check the files for viruses.

- The printed contents of the index-md5.txt file as an appendix.

- The regulatory and information technology points of contact for the submission.

\section{Preparing the media}

CD-ROMs should be packaged carefully to ensure that they arrive in a usable condition. Particularly vulnerable are diskettes and CD-ROM jewel cases shipped in envelopes without bubble-type protective material or stiff backing. The use of a jiffy-type bag by itself to ship media will not provide adequate protection for shipping electronic media.

\section{Transport}

Secure data exchange over the Internet is the recommended means for transporting submissions. However, until the regulatory authorities can develop secure electronic gateways, submissions should continue to be physically transported by courier or registered mail.

\section{Security}

An MD5 checksum should be included for each physical file in the eCTD. The checksum allows the recipient to verify integrity of the physical files in the submission. The XML eCTD DTD provides the location of the files and a tag name contains the checksums.

A checksum of the XML eCTD instance should be included. Applicants should name this checksum file index-md5.txt and include it as a file in the same directory as the XML eCTD instance. Applicants should print the contents of the index-md5.txt file and include the paper copy with the paper cover letter for the submission.

An applicant can provide the eCTD as an encrypted file in accordance with the ICH M2 Recommendation 4.1, if the regulatory body has implemented it. This solution allows the eCTD to be encrypted and transferred over the Internet (if Internet receipt is implemented regionally) or to be encrypted on one of the approved physical media standards. The purpose of encryption is to protect the privacy of the confidential information and to insure it is only available to the authorized receiver. Encryption is always appropriate when the eCTD is sent via the Internet.

Encryption is not considered necessary if the information is sent using a physical media, although encryption is an option. The applicant should assume all liability for the media until it is delivered to the regulatory authority. 
Applicants should not include any file level security settings or password protection for individual files in the eCTD. Applicants should allow printing, changes to the document, selecting text and graphics, and adding or changing notes and form fields. Internal security and access control processes in the regulatory authority should maintain the integrity of the submitted files.

\section{Receipt}

Upon arrival at the regulatory authority, the submission is archived according to local regulations. A read-only copy of the submission is then made available to the review community in the regulatory authority. This is typically done by placing the copy on a network server.

\section{Acknowledgment}

Each regulatory authority should acknowledge the receipt of the eCTD submission according to the policy and procedure of the individual regulatory authority. Applicants should use the address in Table 5-1 to find guidance regarding acknowledgments 


\section{Appendix 6 The eCTD XML Submission}

\section{Background}

There are many factors that have influenced the design of the eCTD. Some that have had a more significant impact on the design are:

- The submissions should accommodate full regulatory dossiers, supplements, amendments and variations.

- The submissions should be able to accommodate regional requirements that are represented in regional guidance documents, regulations, and statutes.

- The technology should be extensible so that as technology changes, the new electronic solutions can be accommodated.

The eCTD is designed around the concept of a backbone. The backbone is similar to a container that holds the files that are part of the submission. The backbone is based on an XML Document Type Definition (DTD). There is a close relationship between the logical documents defined in the CTD and entities in the backbone. The backbone will provide the navigation links to the various files and information that make up the submission.

The file that is produced based on the XML eCTD DTD is the eCTD XML instance or XML backbone. The XML backbone allows more than one entry or link to point to the same physical file. This should be done with caution since it can be more difficult for the regulatory authority to manage the life cycle of that file if there is more than one pointer to the file.

\section{File Names and Directory Structure}

Recipients of the eCTD should be able to directly navigate through the submission at the folder and file level, i.e. without benefit of a customized end user application. The structure of the eCTD and instructions for how to create folder names facilitate this type of navigation.

In order to preserve the navigational linkages that can be present in the documents contained in the eCTD, the directory structure should be preserved by the agencies. The navigational links should be relative links within a module.

Specific folder and file names have been defined in appendix 4. The top-level for the directory structure will vary by region. The identification of the top-level folder uniquely identifies the submission in a region. The submission identification should be used as the folder name in the top-level directory. For example, if the submission number were CTD 123456 , the root directory would be named "ctd-123456". The original submission and subsequent amendments and variations should use the same top-level folder name. Submissions should be differentiated by a subfolder named according to the sequence number of the submission in that region. Table 6-1 and Figure 6-1 illustrate this naming convention. 
Table 6-1

\begin{tabular}{|l|l|l|}
\hline Submission number & Sequence number & Type of submission \\
\hline ctd-123456 & 0000 & Original Submission \\
\hline ctd-123456 & 0001 & $\begin{array}{l}\text { First amendment, } \\
\text { supplement or variation }\end{array}$ \\
\hline ctd-123456 & 0002 & $\begin{array}{l}\text { Second amendment, } \\
\text { supplement or variation }\end{array}$ \\
\hline$\ldots$ & nnnn & $\begin{array}{l}\text { Nth amendment, } \\
\text { supplement or variation }\end{array}$ \\
\hline ctd-123456 &
\end{tabular}

\section{Figure 6-1}

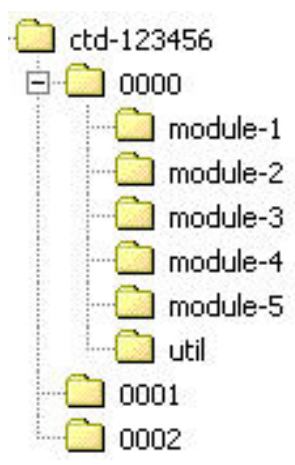

You should submit the xml backbone as a single file named index. $x m l$ which should be placed in the submission sequence number folder for that submission. In the example shown in Figure 6-1, there should be an index.xml file in folder "0000", folder "0001" and folder "0002". The MD5 checksum file, index-md5.txt, should be in each folder with the corresponding index. $x m l$ file. The DTD for index. $x m l$ should be in the "util" folder for each submission.

The regional administrative xml backbone file, if supplied, should be in the region specific module-1 folder for each submission. The DTD for the regional xml backbone file should be in the util folder for each submission.

Table 6-2 presents the file locations for the example in Figure 6-1.

Table 6-2

\begin{tabular}{|l|l|}
\hline Submission Folder & Files \\
\hline ctd-123456/0000 & $\begin{array}{l}\text { index.xml } \\
\text { index-md5.txt }\end{array}$ \\
\hline ctd-123456/0000/module-1/us & us-regional.xml \\
\hline ctd-123456/0000/util & $\begin{array}{l}\text { ich-ectd-1-0.dtd } \\
\text { us-regional-1-0.dtd }\end{array}$ \\
\hline
\end{tabular}




\begin{tabular}{|l|l|}
\hline Submission Folder & Files \\
\hline ctd-123456/0001 & $\begin{array}{l}\text { index.xml } \\
\text { index-md5.txt }\end{array}$ \\
\hline ctd-123456/0001/module-1/us & us-regional.xml \\
\hline ctd-12345/0001/util & $\begin{array}{l}\text { ich-ectd-1-0.dtd } \\
\text { us-regional-1-0.dtd }\end{array}$ \\
\hline ctd-123456/0002 & $\begin{array}{l}\text { index.xml } \\
\text { index-md5.txt }\end{array}$ \\
\hline ctd-123456/0002/module-1/us & us-regional.xml \\
\hline ctd-123456/0002/util & $\begin{array}{l}\text { ich-ectd-1-0.dtd } \\
\text { us-regional-1-0.dtd }\end{array}$ \\
\hline
\end{tabular}

\section{Lifecycle Management}

It is important for the recipients of eCTD to be able to establish the location of the submission in the lifecycle of a product.

The eCTD is capable of containing initial submissions, supplements, amendments and variations. There are no uniform definitions for these terms in the three regions, but amendments and supplements are terms used in the United States. Variations apply in Europe. The variations, supplements and amendments are used to provide additional information to an original regulatory dossier. For example, if a new manufacturer for the drug substance were being proposed, this would result in submission of an amendment or supplement to the FDA and a variation to Europe. When regulatory authorities request additional information, the information is also provided as a variation, supplement or amendment to the original submission. Therefore, the regulatory agencies should have a way to manage the lifecycle for the submission. This function should be provided by each regulatory authority in the form of guidance that can include regional DTDs and specifications. Each regional DTD should be referenced in the eCTD DTD by the submitter.

The eCTD DTD provides some facilities for lifecycle management at the file level. When revisions are sent to a regulatory authority, the new file should be submitted as a leaf element associated with the same tag name as the file being amended or deleted. The "modified-file" attribute of the leaf element should contain the name and relative directory path of the file being amended, replaced or deleted. This will allow the regulatory authority to accurately locate the original file and update the original file's status.

\section{Operation Attribute}

The operation attribute is a key to managing each individual file in a submission. The applicant uses the operation attribute to tell the regulatory authority how the applicant intends the files in the submission to be used. The operation attribute describes the relation between files in subsequent submissions during the life cycle of a medicinal product. In the very first submission all the files will be new. In the second, third, fourth, etc. submission, all the newly submitted files can have different operation 
attributes due to having or not having a relation with previously submitted files. Table 62 describes the meaning of each allowed value of the operation attribute.

Table 6-3 Understanding the Operation Attribute

\begin{tabular}{|l|l|l|l|}
\hline \multicolumn{2}{|c|}{$\begin{array}{c}\text { Operation attribute value } \\
\text { Meaning }\end{array}$} & \multicolumn{2}{|c|}{$\begin{array}{c}\text { What the reviewer might } \\
\text { see when using the Agency } \\
\text { review software }\end{array}$} \\
\cline { 2 - 4 } & $\begin{array}{l}\text { The file has no relationship with files } \\
\text { Nubmitted previously. }\end{array}$ & Current & Previous file \\
\hline Append & $\begin{array}{l}\text { The file itself is new, but due to the relation } \\
\text { this file has with a previously submitted file, } \\
\text { the attribute is "append". The append status is } \\
\text { linked to a previously submitted file on which } \\
\text { this operation has to be executed. The } \\
\text { previously submitted file is indicated by the } \\
\text { "modified file" attribute of the leaf element. }\end{array}$ & Current & $\begin{array}{l}\text { Current - } \\
\text { Appended }\end{array}$ \\
\hline Replace & $\begin{array}{l}\text { The file itself is new, but due to the relation } \\
\text { this file has with a previously submitted file, } \\
\text { the attribute is "replace". The "replace" status } \\
\text { is linked to a previously submitted file on } \\
\text { which this operation is executed. The } \\
\text { previously submitted file is indicated by the } \\
\text { "modified file" attribute of the leaf element. }\end{array}$ & Current & Replaced \\
\hline $\begin{array}{l}\text { There is no new file submitted in this case. } \\
\text { Instead, the leaf has the operation of "delete" } \\
\text { and the "modified-file" attribute identifies the } \\
\text { file in a previous submission that is to be } \\
\text { considered no longer relevant to the review. }\end{array}$ & & \\
\hline Delete & $\begin{array}{l}\text { No longer } \\
\text { relevant to } \\
\text { the review }\end{array}$ & \\
\hline
\end{tabular}

The following cases show examples of the use of each of the operation attribute values. These examples are not a complete list of all possible situations. Consult the appropriate regulatory authority if you have specific questions about the use of the operation attribute.

Case 1 - The first submission of a dossier.

Table 6-4

\begin{tabular}{|l|l|l|l|l|}
\hline $\begin{array}{l}\text { Submission } \\
\text { sequence \# }\end{array}$ & File name & Operation & Modified file & $\begin{array}{l}\text { Sample logical display } \\
\text { in a review tool }\end{array}$ \\
\hline 0000 & $0000 \backslash .$. Istructure.pdf & New & & $\begin{array}{l}\text { Asstructure.pdf } \\
\text { (current) }\end{array}$ \\
\hline
\end{tabular}

Case 2 - Two submissions. Submission 0000 is the first submission of a dossier.

Submission 0001 is a subsequent amendment or variation in which the applicant intends 
to completely replace the structure.pdf file in submission 0000. The intent is to keep the original structure.pdf for historical purposes but to consider only the contents of the $0001 \backslash$ structure.pdf as relevant to the review. These two submissions could be described as follows:

- Submission 0000 is the first submission of the file structure.pdf and this file is the current version of this file.

- Submission 0001, which is submitted at a later time, is the submission of the file structure.pdf, which is now current and replaces the file structure.pdf in submission 0000 .

Table 6-5

\begin{tabular}{|c|c|c|c|c|}
\hline $\begin{array}{l}\text { Submission } \\
\text { sequence \# }\end{array}$ & File name & Operation & Modified file & $\begin{array}{l}\text { Sample logical } \\
\text { display in a review } \\
\text { tool }\end{array}$ \\
\hline 0000 & $0000 \backslash .$. structure.pdf & New & & $\begin{array}{l}\text { Sistructure.pdf } \\
\text { (current) }\end{array}$ \\
\hline 0001 & 0001\.. Istructure.pdf & Replace & 0000\..\structure.pdf & $\begin{array}{l}\text { 垔structure.pdf } \\
\text { (replaced) } \\
\text { 国structure.pdf } \\
\text { (current) }\end{array}$ \\
\hline
\end{tabular}

Case 3 - Two submissions. Submission 0000 is the first submission of a dossier. Submission 0001 is an amendment or variation where the applicant intends to add new information to the original structure.pdf file, which was submitted in submission 0000. The intent is to have the reviewer consider the contents of both files relevant to the submission. These two submissions could be described as follows:

- Submission 0000 is the first submission of the file structure.pdf and this file is the current version of this file.

- Submission 0001, which is submitted at a later time, is the submission of the file structure.pdf, which is the current file but contains information that should be appended to file structure.pdf in submission 0000. Both files should be considered relevant to the review of the dossier.

Table 6-6

\begin{tabular}{|c|c|c|c|c|}
\hline $\begin{array}{l}\text { Submission } \\
\text { sequence \# }\end{array}$ & File name & Operation & Modified file & $\begin{array}{l}\text { Sample logical } \\
\text { display in a review } \\
\text { tool }\end{array}$ \\
\hline 0000 & 0000\..\structure.pdf & New & & $\begin{array}{l}\text { Istructure.pdf } \\
\text { (current) }\end{array}$ \\
\hline 0001 & $0001 \backslash .$. structure.pdf & Append & $0000 \backslash .$. structure.pdf & $\begin{array}{l}\text { Astructure.pdf } \\
\text { (current - } \\
\text { appended) }\end{array}$ \\
\hline
\end{tabular}




\begin{tabular}{|l|l|l|l|l|}
\hline & & & & $\begin{array}{l}\text { gsstructure.pdf } \\
\text { (current) }\end{array}$ \\
\hline
\end{tabular}

Case 4 - Two submissions. Submission 0000 is the first submission of a dossier. Submission 0001 is an amendment or variation where the applicant intends to delete a file in the previous submission. The intent is to have the reviewer disregard the contents of the original file, possibly because it should not have been submitted with the original dossier. These two submissions could be described as follows:

- Submission 0000 is the first submission of the file structure.pdf and this file is the current version of this file.

- Submission 0001, which is submitted at a later time, requests that the file structure.pdf in submission 0000 be deleted and no longer considered relevant to the review of the dossier.

Table 6-7

\begin{tabular}{|c|c|c|c|c|}
\hline $\begin{array}{l}\text { Submission } \\
\text { sequence \# }\end{array}$ & File name & Operation & Modified file & $\begin{array}{l}\text { Sample logical } \\
\text { display in a review } \\
\text { tool }\end{array}$ \\
\hline 0000 & 0000\..\structure.pdf & New & & $\begin{array}{l}\text { Isstructure.pdf } \\
\text { (current) }\end{array}$ \\
\hline 0001 & & Delete & 0000\..\structure.pdf & $\begin{array}{l}\text { Sistructure.pdf } \\
\text { (no longer } \\
\text { relevant to the } \\
\text { review) }\end{array}$ \\
\hline
\end{tabular}

\section{DTD Content Model}

The content model of the eCTD is derived from the organization of the Common Technical Document. The graphic representation of a portion of the content model is shown below. The content model is hierarchical starting at the "ectd" and going down to a specific item to be included in the submission. This example shows how the section of the CTD containing summaries is structured. 


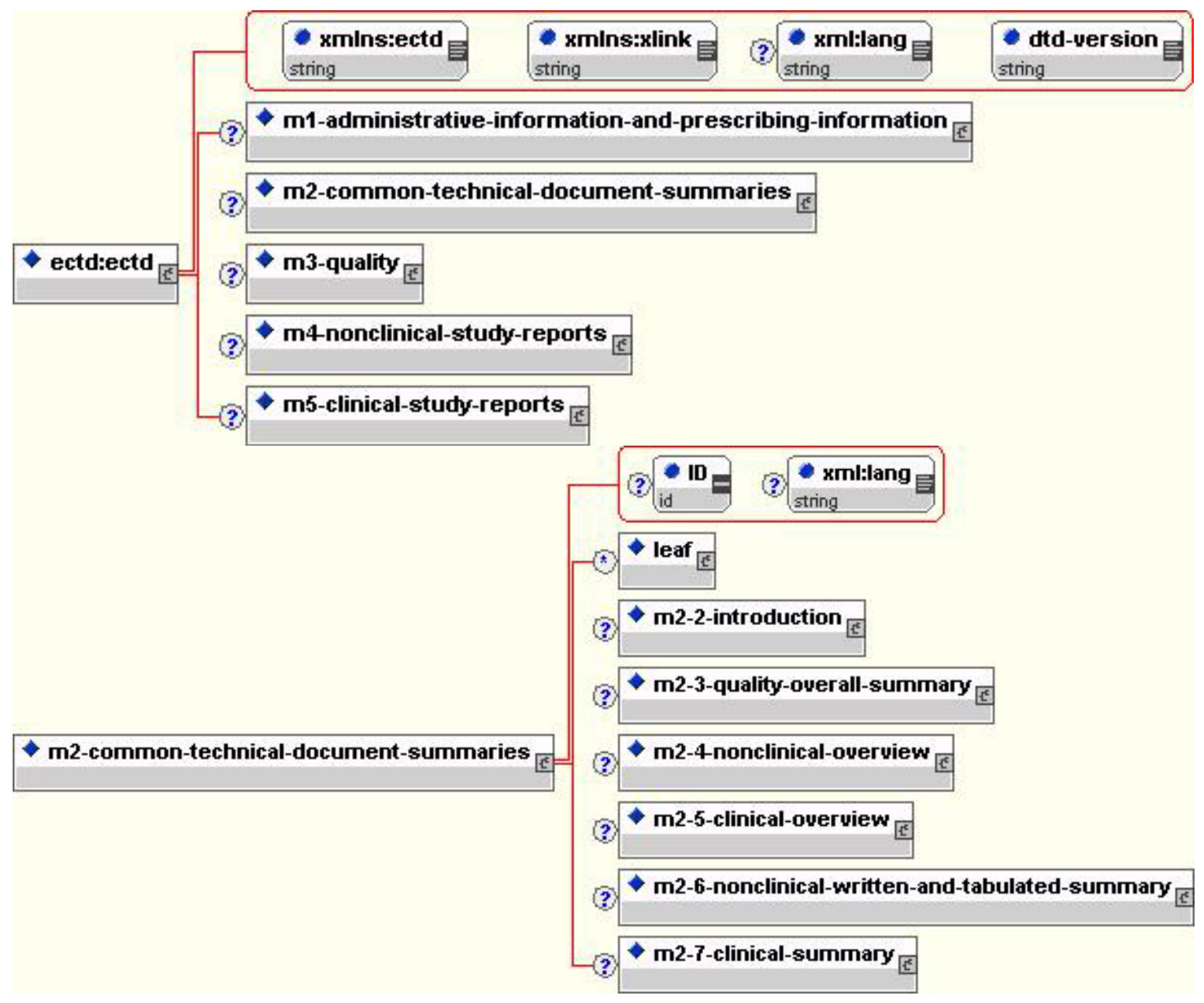

Once the appropriate tag has been selected, use the $<$ leaf $>$ element and attributes to specify a file in the submission. See "Instructions for preparing the eCTD" in this appendix for details.

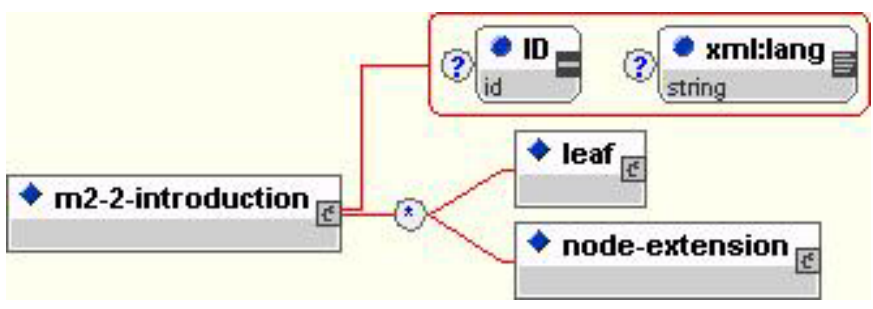




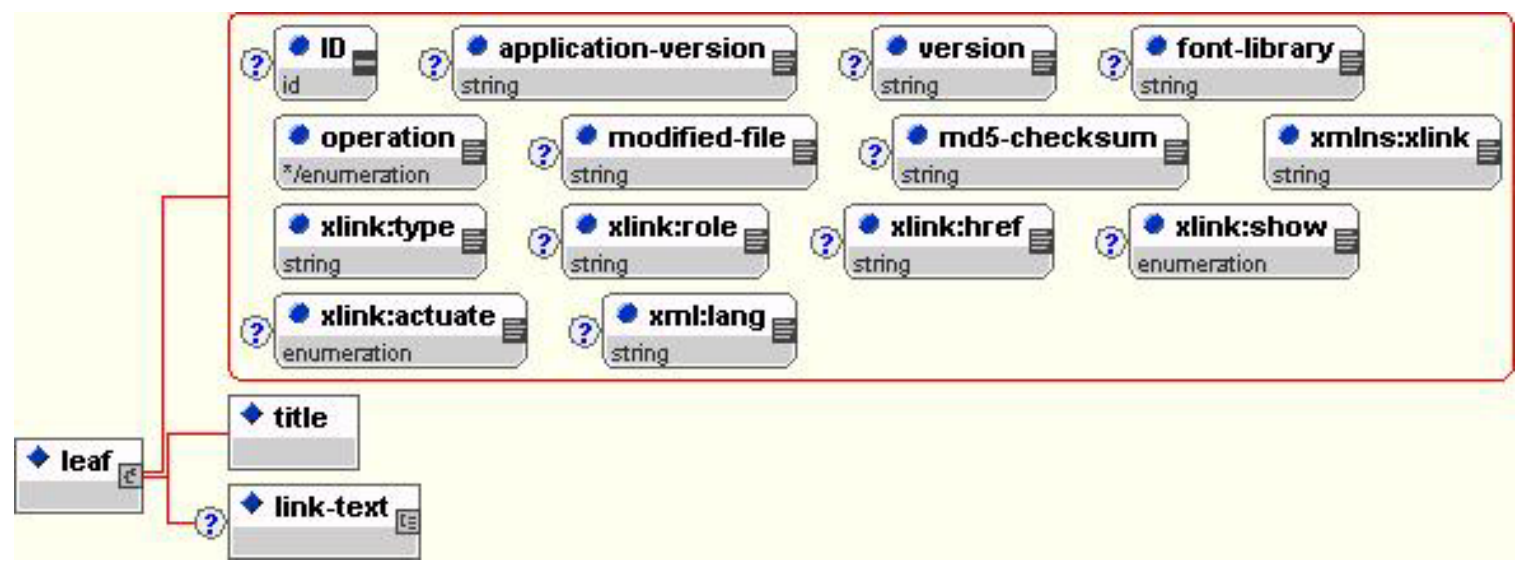

Page 6-8 


\section{eCTD Element/Attribute Instructions}

The eCTD consists of 5 primary sub modules:

- m1-administrative-information-and-prescribing-information

- m2-common-technical-document-summaries

- m3-quality

- m4-nonclinical-study-reports

- m5-clinical-study-reports

Each of the first 5 sub modules is further decomposed into sub-elements, each with a distinct $<$ tag $>$ that represents a CTD table of contents location. The steps should be completed as shown in the following example, where all files are submitted for modules 1 through 5:

1. You should select a tag element that best corresponds to the CTD table of contents location for a document or file being submitted. For example, select the tag $<\mathrm{m} 2-4-$ nonclinical-overview $>$ to submit the nonclinical overview document.

2. You should create a child $<$ leaf $>$ element underneath the $<$ m2-4-nonclinicaloverview $>$ tag.

3. You should provide the relative location and file name of the actual file containing the nonclinical overview in the "xlink:href" attribute for the $<$ leaf $>$ element.

4. You should provide a descriptive title for the file that contains the nonclinical overview in the $<$ title $>$ element of the $<$ leaf $>$.

5. You should provide information for the appropriate attributes of the $<$ leaf $>$ element as described in Table 6-3.

The following table describes each of these elements and attributes in further detail.

Table 6-8

\begin{tabular}{|l|l|l|l|}
\hline Element & Attribute & Description/Instructions & Example \\
\hline $\begin{array}{l}\text { Any table of } \\
\text { contents tag } \\
\text { such as }<\mathrm{m} 2-4- \\
\text { nonclinical- } \\
\text { overview }>\end{array}$ & $\begin{array}{l}\text { A table of contents tag represents a } \\
\text { grouping of one or more files related } \\
\text { to a specific section of the Common } \\
\text { Technical Document. } \\
\text { One or more child }<\text { leaf }>\text { elements } \\
\text { can be declared for a parent table of } \\
\text { contents tag. } \\
\text { It is possible to extend a table of } \\
\text { contents tag by providing a }<\text { node- } \\
\text { extension> element. This can be } \\
\text { done at the lowest level of the }\end{array}$ & \\
& &
\end{tabular}




\begin{tabular}{|c|c|c|c|}
\hline \multirow[t]{4}{*}{ Element } & Attribute & Description/Instructions & Example \\
\hline & & $\begin{array}{l}\text { defined table of contents tags but } \\
\text { should be done only when absolutely } \\
\text { necessary. See the section } \\
\text { "Instructions for extending eCTD tag } \\
\text { elements" in this appendix. }\end{array}$ & \\
\hline & ID & $\begin{array}{l}\text { A unique identifier for this location } \\
\text { in the XML instance. }\end{array}$ & \\
\hline & xml:lang & $\begin{array}{l}\text { The primary language used by the } \\
\text { files in this entire section of the } \\
\text { submission. Use ISO-639 standard } \\
\text { language abbreviations }\end{array}$ & en \\
\hline \multirow[t]{8}{*}{$<$ leaf $>$} & & $\begin{array}{l}\text { A leaf corresponds to a file. } \\
\text { One or more child leaf elements can } \\
\text { be submitted for a parent table of } \\
\text { contents tag. }\end{array}$ & \\
\hline & \begin{tabular}{|l} 
application- \\
version
\end{tabular} & $\begin{array}{l}\text { The version of the software } \\
\text { application that was used to create } \\
\text { this file. }\end{array}$ & Acrobat 5 \\
\hline & Font-library & $\begin{array}{l}\text { The commercial name of the font or } \\
\text { font library needed to properly view } \\
\text { the submitted file. }\end{array}$ & \\
\hline & ID & $\begin{array}{l}\text { Unique identifier for this location in } \\
\text { the XML instance. }\end{array}$ & \\
\hline & checksum & $\begin{array}{l}\text { The checksum value for the file } \\
\text { being submitted. }\end{array}$ & $\begin{array}{l}\text { e854d3002c02a61 fe5cbe926fd } 97 b \\
001\end{array}$ \\
\hline & $\begin{array}{l}\text { checksum- } \\
\text { type }\end{array}$ & The checksum algorithm used. & MD5 \\
\hline & modified-file & $\begin{array}{l}\text { The name of the file to be modified } \\
\text { as indicated in the "operation" } \\
\text { attribute. This file name should } \\
\text { include the relative path to the file. } \\
\text { If no file is being modified, then you } \\
\text { should not supply the "modified-file" } \\
\text { attribute. }\end{array}$ & $\begin{array}{l}\text { /0000/module- } 2 / \text { clinical- } \\
\text { summary/references.pdf }\end{array}$ \\
\hline & operation & $\begin{array}{l}\text { Indicates the operation to be } \\
\text { performed on the "modified-file". } \\
\text { You should select one of the } \\
\text { following valid values: } \\
\text { new } \\
\text { replace } \\
\text { append } \\
\text { delete } \\
\text { See the section Operation Attribute } \\
\text { in this appendix for details on the } \\
\text { meaning of these values. }\end{array}$ & new \\
\hline
\end{tabular}




\begin{tabular}{|c|c|c|c|}
\hline Element & Attribute & Description/Instructions & Example \\
\hline & Version & $\begin{array}{l}\text { The file submitter's internal version } \\
\text { number or version identification for } \\
\text { the report. }\end{array}$ & V23.5 \\
\hline & xlink:actuate & Not Currently Used & \\
\hline & xlink:href & $\begin{array}{l}\text { Provide the pointer to the actual file. } \\
\text { Use the relative path to the file and } \\
\text { the file name. }\end{array}$ & $\begin{array}{l}\text { module-2/clinical- } \\
\text { summary/references.pdf }\end{array}$ \\
\hline & xlink:role & Not Currently Used & \\
\hline & xlink:show & Not Currently Used. & \\
\hline & xlink:type & Fixed value of "simple". & simple \\
\hline \multirow[t]{2}{*}{$<$ title $>$} & & $\begin{array}{l}\text { This element is associated with a } \\
\text { "leaf" and provides a description of } \\
\text { the file being submitted. }\end{array}$ & study report 1234 \\
\hline & ID & $\begin{array}{l}\text { Unique identifier for this location in } \\
\text { the XML instance }\end{array}$ & ID050520 \\
\hline
\end{tabular}

\section{Instructions for a Simple New Submission}

The following XML fragment demonstrates the submission of a clinical overview of efficacy as a single PDF document.

$<? \mathrm{xml}$ version $=" 1.0 "$ encoding $=$ "UTF-8"? $>$

$<$ !DOCTYPE ectd:ectd SYSTEM "util/dtd/ich-ectd-1-0.dtd">

<ectd:ectd xmlns:ectd = "http://www.ich.org/ectd" xmlns:xlink = "http://www.w3c.org/1999/xlink"> $<\mathrm{m} 2$-common-technical-document-summaries $>$ $<\mathrm{m} 2-5$-clinical-overview $>$

$<\mathrm{m} 2-5-4-o v e r v i e w-o f-e f f i c a c y ~ x m l: l a n g=" e n ">$ $<$ leaf operation $=$ "new" xlink:type $=$ "simple" checksum $=$ "e854d3002c02a61fe5cbe926fd97b001" xlink:href = "module-2/clinical-summary/efficacy-overview.pdf" application-version $=$ "Acrobat $5 ">$ $</$ leaf $>$

$<$ title $>$ Overview of efficacy $</$ title $>$

$</$ m2-5-4-overview-of-efficacy $>$

$</ \mathrm{m} 2-5$-clinical-overview $>$

$</$ ectd:ectd $>$

$</$ m2-common-technical-document-summaries $>$

This submission includes the file "efficacy-overview.pdf" in the relative directory "module-2/clinical-summary" (i.e. the one starting below the Dossier number and submission sequence directories). The file is "new" and has a descriptive name of "Overview of efficacy"

The regional review application should treat this as a new submission to be associated with the submission identified in CTD module 1, which is region specific.

If this is the first submission for Dossier CTD 123456, all the files in this submission are in the ctd-123456\0000 directory and below. 


\section{Instructions for an Amendment, Supplement or Variation}

In the previous example, an efficacy overview was submitted. In this example, it is replaced by an updated version.

To replace a file, add the replacement file $<$ leaf $>$ element under the same tag element as the original file. If this is the second submission for Dossier CTD 123456, all the files in this submission are in the ctd-123456\0001 directory and below.

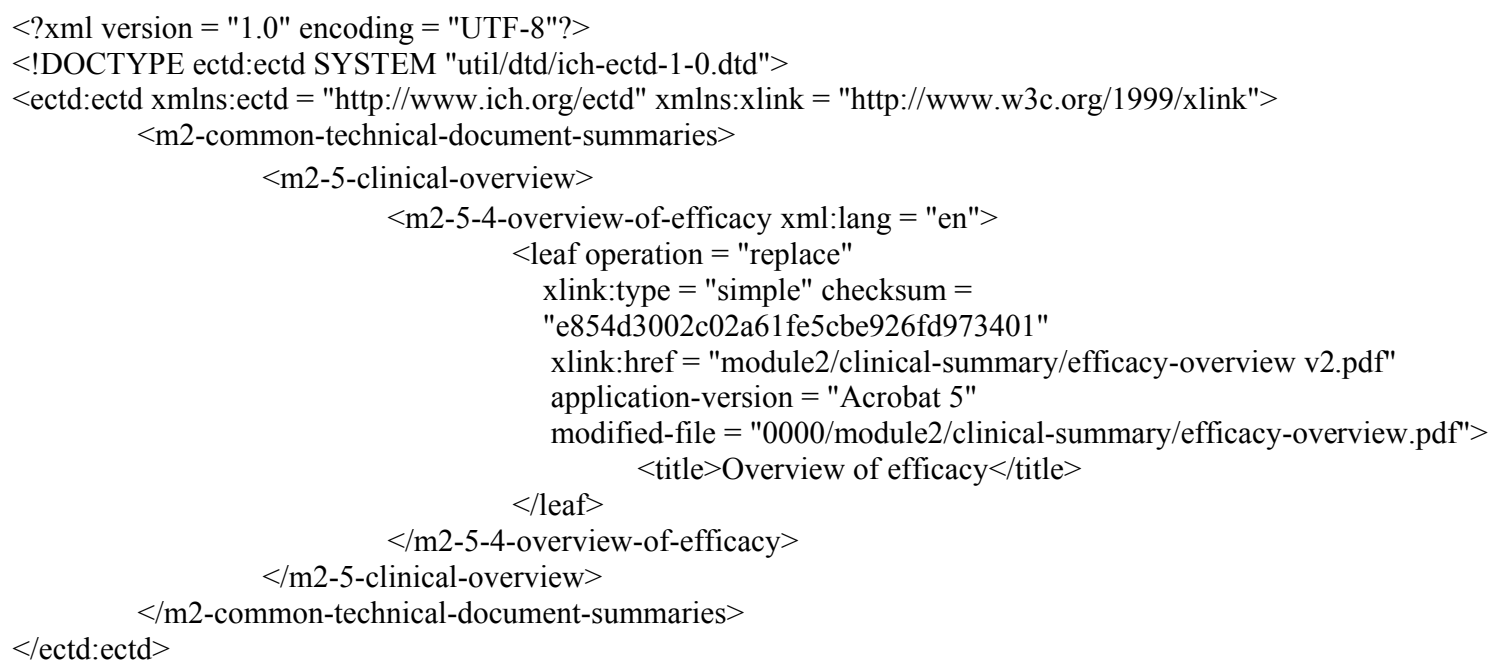

\section{Instructions for Multiple Indications ${ }^{7}$}

Multiple therapeutic indications use an additional attribute associated with the $<$ m2-7-3summary-of-clinical-efficacy $>$ and the $<\mathrm{m} 5$-3-5-reports-of-efficacy-and-safety-studies $>$ elements to allow multiple indications to be submitted. The following table shows the use of these attributes.

Table 6-9

\begin{tabular}{|l|l|l|l|}
\hline \multicolumn{1}{|c|}{ Element } & \multicolumn{1}{|c|}{ Attribute } & \multicolumn{1}{c|}{ Description/Instructions } & \multicolumn{1}{c|}{ Example } \\
\hline $\begin{array}{l}<\mathrm{m} \text { 2-7-3-summary- } \\
\text { of-clinical-efficacy }>\end{array}$ & Indication & Name of the indication & pain \\
\hline $\begin{array}{l}<\mathrm{m} 5-3-5-\text {-reports-of- } \\
\text { efficacy-and-safety- } \\
\text { studies }>\end{array}$ & Indication & Name of the indication. & pain \\
\hline
\end{tabular}

Note that the indication attribute is used by the regulatory authority to apply to all the table of contents tags beneath the $<$ m2-7-3-summary-of-clinical-efficacy $>$ and $<$ m5-3-5reports-of-efficacy-and-safety-studies $>$ tags. This is an example of the a section of the instance showing the submission of information about two indications:

$<? \mathrm{xml}$ version $=" 1.0 "$ encoding $=$ "UTF-8"?>

$<$ !DOCTYPE ectd:ectd SYSTEM "util/dtd/ich-ectd-1-0.dtd">

<ectd:ectd xmlns:ectd = "http://www.ich.org/ectd" xmlns:xlink = "http://www.w3c.org/1999/xlink">

\footnotetext{
${ }^{7}$ Note that these XML examples are examples only and do not necessarily contain all of the elements and attributes that you should use when preparing an eCTD submission.
} 


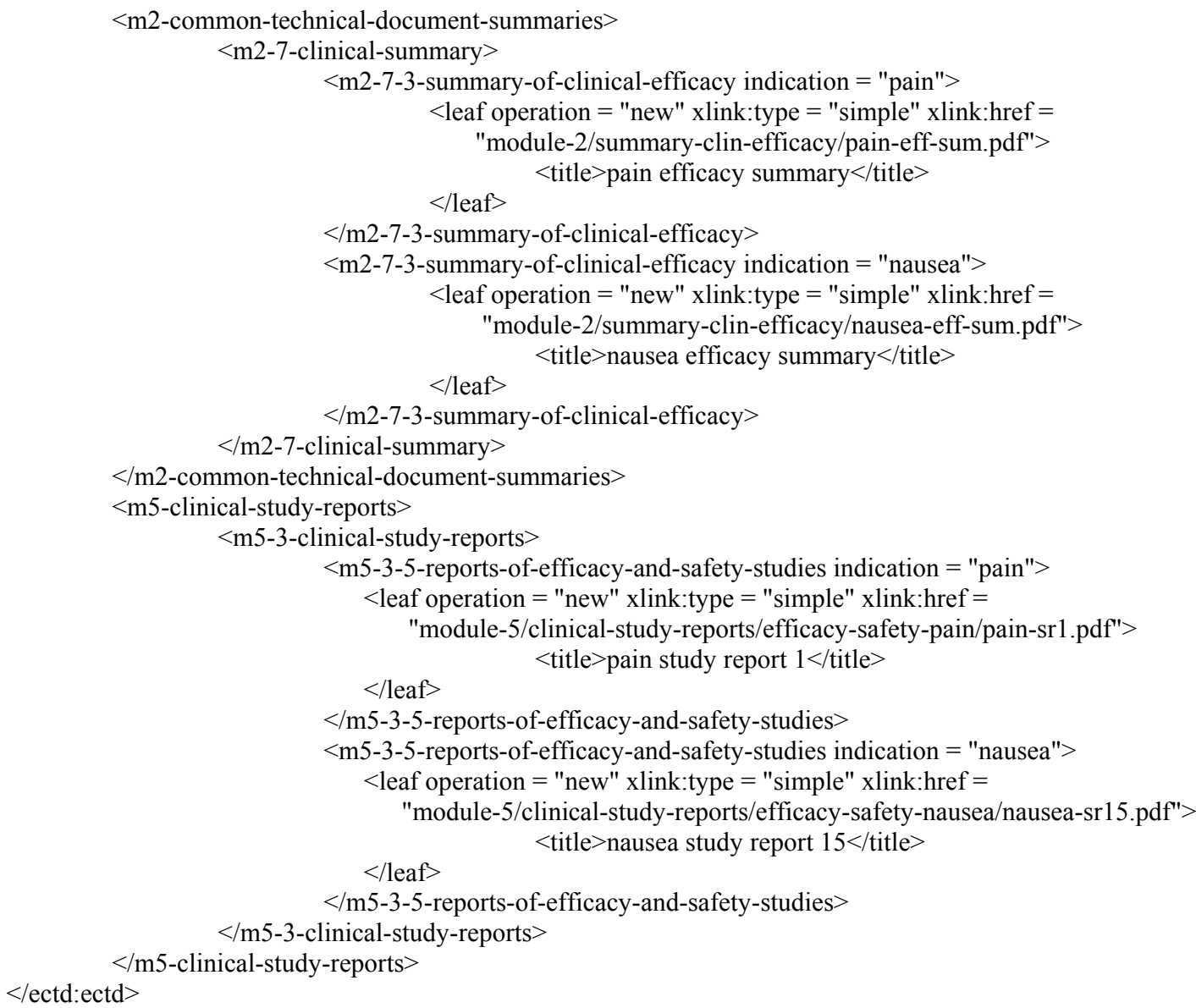

\section{Instructions for Multiple Drug Substances, Manufacturers and Products}

Multiple drug substances use additional attributes associated with the $<\mathrm{m} 3-2$-s-drugsubstance $>$ element to allow unique combinations of the drug substance name and manufacturer to be submitted. The following table shows the use of these attributes.

Table 6-10

\begin{tabular}{|l|l|l|l|}
\hline \multicolumn{1}{|c|}{ Element } & \multicolumn{1}{|c|}{ Attribute } & \multicolumn{1}{|c|}{ Description/Instructions } & \multicolumn{1}{|c|}{ Example } \\
\hline $\begin{array}{l}\text { <m3-2-s-drug- } \\
\text { substance }>\end{array}$ & Substance & Name of one of the drug substances & Acetaminophen \\
\hline & Manufacturer & $\begin{array}{l}\text { Name of the manufacturer of the } \\
\text { drug substance }\end{array}$ & my supplier \\
\hline
\end{tabular}

This is an example of the a section of the instance showing the submission of information about two drug substances, one of which is supplied by two manufacturers:

$<\mathrm{m} 3$-2-body-of-data $>$

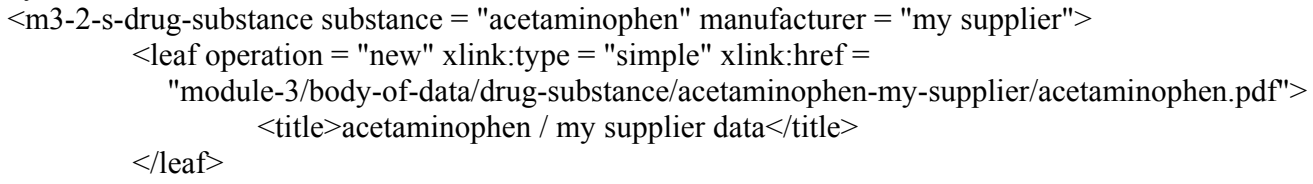




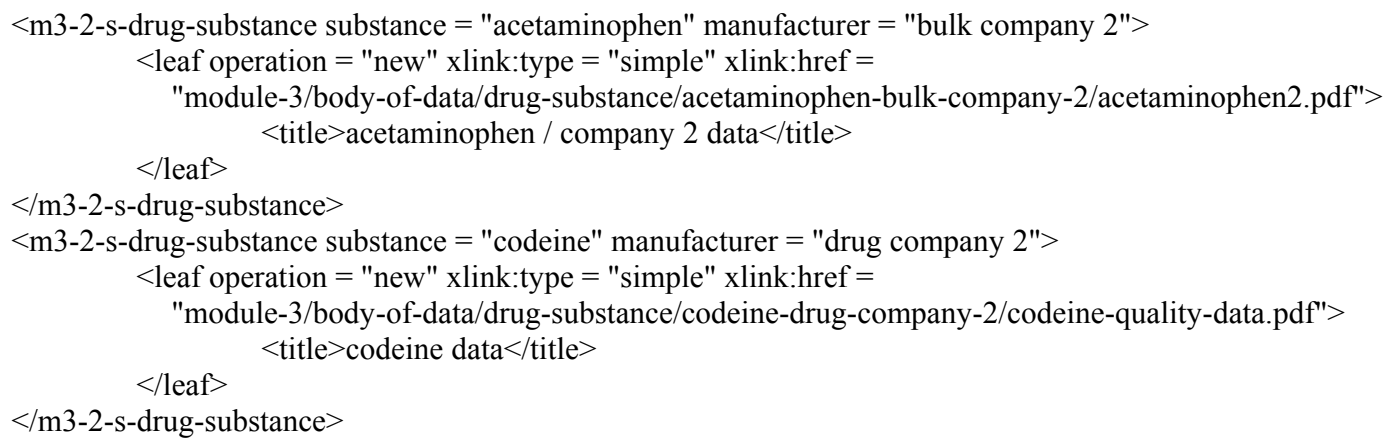

Multiple drug products use additional attributes associated with the $<\mathrm{m} 3$-2-p-drugproduct $>$ element to allow unique combinations of the drug product name and dosage form to be submitted. The following table shows the use of these attributes.

Table 6-11

\begin{tabular}{|l|l|l|l|}
\hline \multicolumn{1}{|c|}{ Element } & \multicolumn{1}{|c|}{ Attribute } & \multicolumn{1}{|c|}{ Description/Instructions } & \multicolumn{1}{|c|}{ Example } \\
\hline $\begin{array}{l}\text { <m3-2-p-drug- } \\
\text { product }>\end{array}$ & Product-name & Name of one of the drug products & Wonder drug \\
\hline & Dosageform & $\begin{array}{l}\text { Name of the dosage form of the drug } \\
\text { product }\end{array}$ & Capsules \\
\hline
\end{tabular}

This is an example of a section of the instance showing the submission of information about two drug products:

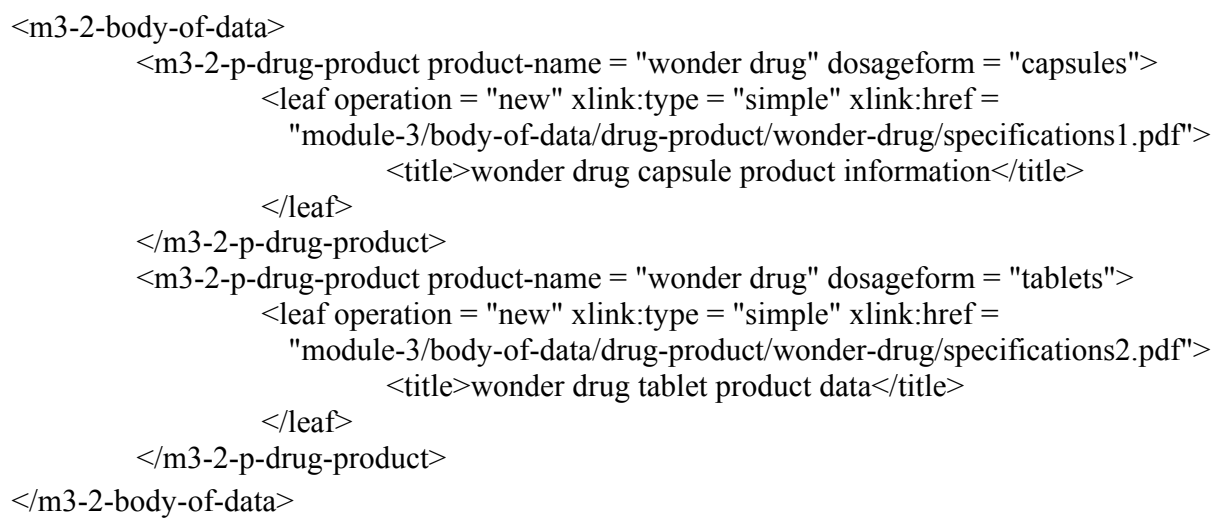

\section{Instructions for extending XML eCTD DTD elements}

An applicant can extend the definition of an element by creating node extensions beneath a defined table of contents tag. Using node extensions is discouraged and should only be done when there is no other feasible means to submit information. The child element $<$ node-extension $>$ should be used for each new table of contents node created. The $<$ title $>$ element value is inherited from the parent element. You should follow the following principles when using $<$ node-extension $>$ :

1. You should only extend the lowest level of defined elements. For example you can extend the $<\mathrm{m} 2$-3-r-regional-information $>$ element but not the $<\mathrm{m} 2$-3-quality- 
overall-summary> element since the latter is not the lowest element defined in the table of contents.

2. Do not extend the element more than one level. For example, you should not extend $<$ node-extension $><$ title $>$ special-fda-summary $</$ title $><$ /node-extension $>$ with another $<$ node-extension $>$.

The following is an example of a section of the eCTD instance in which an applicant extends the $<\mathrm{m} 2-3$-r-regional-information $>$ to provide specific regional information as requested by a regulatory authority. The title element associated with the $<$ nodeextension $>$ describes the extension. Alternatively, the regional information in this example could have been provided as a $<$ leaf $>$ element under the $<$ m2-3-r-regionalinformation> element without the use of a "node extension".

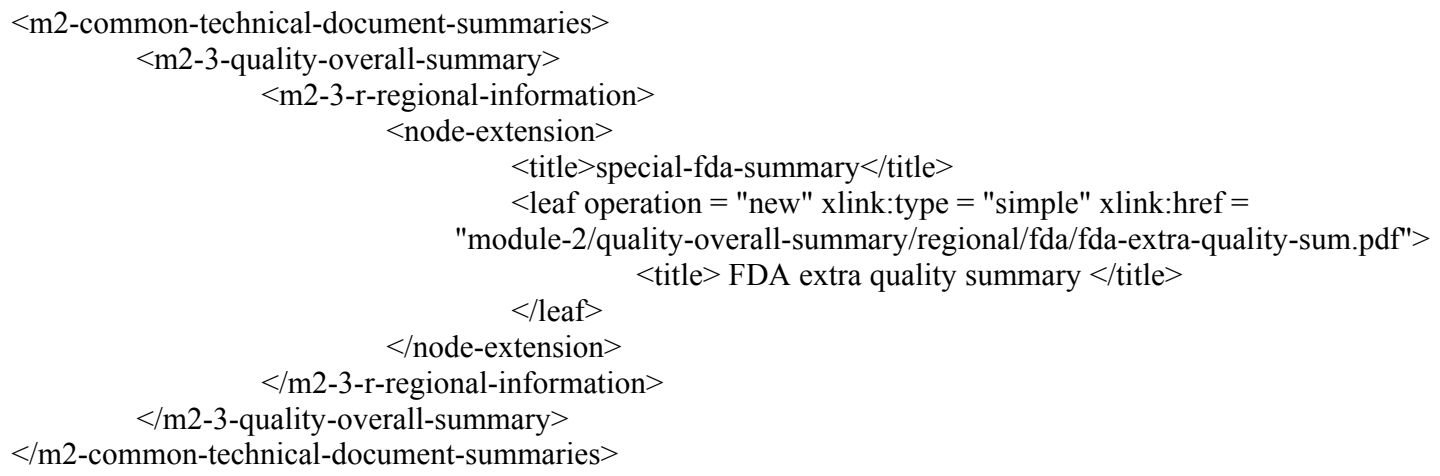

To update a file that has been submitted as an extended node, you should submit the replacement file using exactly the same element and "node extension" information, including the $<$ title $>$ element for the $<$ node-extension $>$. This makes it possible for the regulatory authority to locate the original file and update its status.

\section{Instructions for Submitting Sections as Paper}

During the transition to fully electronic submissions of the CTD, some sections can be submitted as paper only. These sections should be identified in the XML eCTD instance by including a PDF file in the instance that describes the content and location of the paper section. For example, the PDF file might consist of only one page with the name of the CTD document and the physical volume number and tab identifier. The $<$ title $>$ element in the XML eCTD instance could indicate that this is a paper submission.

This is an example of the instance showing the submission of a paper efficacy overview document.

$<\mathrm{m} 2-5$-clinical-overview $>$

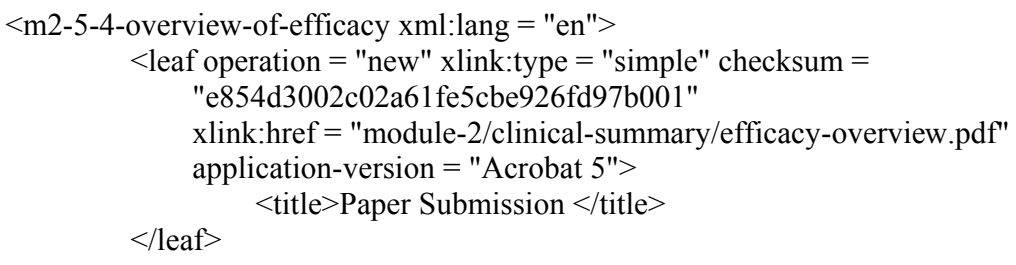


$</$ m2-5-4-overview-of-efficacy $>$

$</$ m2-5-clinical-overview $>$

Page 6-16 


\section{Appendix 7 Specification for Submission Formats}

\section{Introduction}

This appendix describes the way in which files should be constructed for inclusion in the eCTD. The file formats included in this section are those formats that are commonly used in electronic submissions. Other formats can be used according to guidance published in each region.

\section{PDF}

Adobe Portable Document Format (PDF) is a published format created by Adobe Systems Incorporated (http://www.adobe.com). It is not necessary to use a product from Adobe or from any specific company to produce PDF documents. PDF is accepted as a standard for documents defined in this specification. The following recommendations support the creation of PDF files that Agencies can review effectively. For any specification of the Japanese version of Adobe Acrobat, or where Japanese characters will be in the file, please refer to the regional guidance.

To ensure that PDF files can be accessed efficiently, PDF files should be no larger than 50 Megabytes. The files should be saved "optimized" as shown in figure 7-1.

Figure 7-1

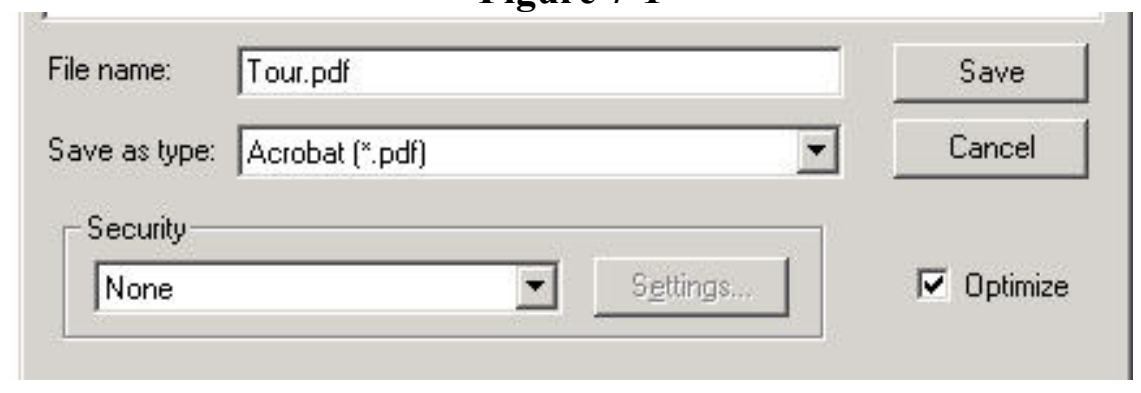

\section{Version}

Agencies should be able to read all PDF files with version 4.0 or higher of the Acrobat Reader. Agencies should not need any additional software to read and navigate the PDF files. However, review can be facilitated through use of Adobe Acrobat since significantly more functionality is available in this product than with Acrobat Reader.

\section{Fonts}

PDF viewing software automatically substitutes a font to display text if the font used to create the text is unavailable on the reviewer's computer. Font substitution can affect a document's appearance and structure, and in some cases, it can affect the information conveyed by a document. Agencies cannot guarantee the availability of any fonts except Times New Roman, Arial and Courier and fonts supported in the Acrobat product set itself. Therefore, all additional fonts used in the PDF files should be embedded to ensure that those fonts would always be available to the reviewer. When embedding fonts, all 
characters for the font should be embedded, not just a subset of the fonts being used in the document.

One problem associated with embedding fonts is that embedding requires additional computer storage space. Three techniques to help limit the storage space taken by embedding fonts include:

- Limiting the number of fonts used in each document

- Using only True Type or Adobe Type 1 fonts

- Avoiding customized fonts

Resizing a document because the contents are too small to read is inefficient. Times New Roman, 12-point font, the font used for this document is adequate in size for reading narrative text and should be used whenever possible. It is sometimes tempting to use fonts which are smaller than 12 point in tables and charts but this should be avoided whenever possible. When choosing a point size for tables, a balance should be made between providing sufficient information on a single page that may facilitate data comparisons for the reviewer while still achieving a point size that remains legible. The corollary of this is that in making point size larger, more tables might be necessary, which can complicate data comparisons for a reviewer since data might now be included in separate tables. Generally, point sizes 9-10 are considered acceptable in tables but smaller point sizes should be avoided.

\section{Use of Color fonts}

The use of a black font color is recommended. Blue font can be used for hypertext links. If a font color other than black is used, light colors that do not print well on grayscale printers should be avoided. Color reproduction can be tested prior to submission by printing sample pages from the document using a gray scale printer. The use of background shadowing should be avoided.

\section{Page Orientation}

Pages should be properly oriented so that all portrait pages are presented in portrait and all landscape pages are presented in landscape. To achieve this, the page orientation of landscape pages should be set to landscape prior to saving the PDF document in final form.

\section{Page Size and Margins}

The print area for pages should fit on a sheet of A4 or Letter paper. A sufficient margin (at least $2.5 \mathrm{~cm}$ ) on the left side of each page should be provided in order to avoid obscuring information if the reviewer subsequently prints and binds the pages for temporary use. For pages in landscape orientation (typically tables and publications) smaller margins are allowable (at least $2.0 \mathrm{~cm}$ at the top and $0.8 \mathrm{~cm}$ left and right) so as to allow more information, displayed legibly, on the page (see Section 3, Fonts). It is acceptable that header and footer information appears within these margins but not so close to the page edge that it may risk being lost upon printing. 


\section{Source of Electronic Document}

PDF documents produced by scanning paper documents are usually inferior to those produced from an electronic source document. Scanned documents are more difficult to read and do not allow reviewers to search or copy and paste text for editing. They should be avoided where possible.

\section{Methods for Creating PDF Documents and Images}

The method used for creating PDF documents should produce the best replication of a paper document. To ensure that the paper and PDF version of the document are the same, the document should be printed from the PDF version. Documents that are available only in paper should be scanned at resolutions that will ensure the pages are legible both on the computer screen and when printed. At the same time, the file size should be limited. It is recommended that scanning be undertaken at a resolution of 300 dots per inch (dpi) to balance legibility and file size. The use of grayscale or color is discouraged because of file size. After scanning, resampling to a lower resolution should be avoided.

When creating PDF files containing images, the images should not be downsampled. Downsampling does not preserve all of the pixels in the original. For PDF images, one of the following lossless compression techniques should be used:

- For lossless compression of color and grayscale images, use Zip/Flate (one technique with two names). This is specified in Internet RFC 1950 and RFC 1951 (http://info.internet.isi.edu/in-notes/rfc/files/rfc1950.txt).

- For lossless compression of black and white images, use the CCITT Group 4 Fax compression technique. It is specified as CCITT recommendations T.6 (1988) Facsimile coding schemes and coding control functions for Group 4 facsimile apparatus.

Paper documents containing hand-written notes should be scanned at 300 dpi. Handwritten notes should be done in black ink for clarity.

For photographs, the image should be obtained with a resolution of $600 \mathrm{dpi}$. If black and white photos are submitted, 8-bit grayscale images should be considered. If color photos are submitted, 24-bit RGB images should be considered. A captured image should not be subjected to non-uniform scaling (i.e., sizing).

Gels and karyotypes should be scanned directly, rather than from photographs. Scanning should be at 600 dpi and 8-bit grayscale depth.

Plotter output graphics should be scanned or captured digitally at 300 dpi.

High-pressure liquid chromatography or similar images should be scanned at 300 dpi. Applicants should validate the quality of the renditions. 


\section{Hypertext Linking and Bookmarks}

Hypertext links and bookmarks are techniques used to improve navigation through PDF documents. Hypertext links can be designated by rectangles using thin lines or by blue text.

In general, for documents with a table of contents, bookmarks for each item listed in the table of contents should be provided including all tables, figures, publications, other references, and appendices. These bookmarks are essential for the efficient navigation through documents. In general, including a bookmark to the main table of contents for a submission or module is helpful. The bookmark hierarchy should be made identical to the table of contents with no additional bookmark levels beyond those present in the table of contents.

Each additional level increases the need for space to read the bookmarks. The use of no more than 4 levels in the hierarchy is recommended.

Hypertext links throughout the body of the document to supporting annotations, related sections, references, appendices, tables, or figures that are not located on the same page are helpful and improve navigation efficiency. Relative paths should be used when creating hypertext links to minimize the loss of hyperlink functionality when folders are moved between disk drives. Absolute links that reference specific drives and root directories will no longer work once the submission is loaded onto the Agency's network servers.

When creating bookmarks and hyperlinks, the magnification setting Inherit Zoom should be used so that the destination page displays at the same magnification level that the reviewer is using for the rest of the document.

\section{Page Numbering}

If a submission includes more than one document, no additional volume or page numbering is necessary. Only page numbers for individual documents are needed. It is easier to navigate through an electronic document if the page numbers for the document and the PDF file are the same. To accomplish this, the initial page of the document should be numbered page 1, with no use of Roman numerals or unnumbered pages in the document. If this is not done, Acrobat Reader would include such numbering within its page count and thus put the Acrobat numbering out of synchrony with the internal document page numbers.

Two exceptions to this rule can occur, details of which can be found in the guidance for the modules of the CTD.

- Firstly, where a document is split because of its size (e.g. >50MB), under which circumstances the second or subsequent file should be numbered consecutively to that of the first or preceding file.

- Secondly, where several small documents with their own internal page numbering have been brought together into a single file, under which circumstances it is not 
considered necessary to provide additional page numbering, but the start of each sub-document should be book marked.

\section{Document Information Fields}

Document information fields should not be used for the common portions of the eCTD, but they may be appropriate for some of the regional documents. Recommendations for the document information fields will be provided in the regional guidance for the specific submission type.

\section{Open Dialog Box}

The open dialog box sets the document view when the file is opened. The initial view of the PDF files should be set as Bookmarks and Page. If there are no bookmarks, the initial view as Page only should be set. The Magnification and Page Layout should be set as default.

\section{Security}

No security settings or password protection for PDF files should be included. Security fields should be set to allow printing, changes to the document, selecting text and graphics, and adding or changing notes and form fields.

\section{Indexing PDF Documents}

Full text indices can be used to help find specific documents and/or search for text within documents. When a document or group of documents is indexed, all words and numbers in the file and all information stored in the Document Information fields are stored in special index files that are functionally accessible using the search tools available in Acrobat. Portions of a document that are imaged are not indexed. Even if the document only contains images, the text in the Document Information fields of the file will be indexed.

These full text indices should not be confused with a table of contents. Adobe Acrobat Catalog is one example of a tool that can be used to index PDF documents. Indices should not require extensions or additions to off-the-shelf Acrobat programs.

Further recommendations for full text indices will be provided in regional guidance.

\section{Use of Acrobat Plug-Ins}

It is considered acceptable to use plug-ins to assist in the creation of a submission. However, the review of the submission should not require the use of any plug ins, in addition to those provided with Adobe Acrobat because Agencies should not be required to archive additional plug-in functionality. 


\section{XML Files}

A working group at the World Wide Web Consortium (W3C) developed XML. It is a nonproprietary language developed to improve on previous mark up languages including standard generalized markup language (SGML) and hypertext markup language (HTML).

Information in an XML file is divided into specific pieces. These pieces are called objects or element types. The element type identifies the piece of information. For example, the name of the company submitting a registration application in ECTD format for review is identified with the element type <applicant $>$. All element type names are bracketed using the special characters $<>$. Inside the XML document, the element type name is placed just prior to the piece of information and after the information. This is called tagging. So, in the XML file, the applicant could be tagged as follows $<$ applicant $>$ Worldwide Pharmaceuticals Inc. $<$ /applicant $>$. The / prior to the element type denotes that this is the end of the information about the applicant.

By using a hierarchical structure, XML allows you to relate two or more elements. This is accomplished by nesting one element within another.

Additional information about the element type is provided by attributes. Attributes are placed within the element types and are surrounded by " ". For example, if you wanted to show that the applicant name is presented in the English language, you could add this piece of information as an attribute. This could be represented in the XML file as $<$ applicant XML:LANG="EN"> Worldwide Pharmaceuticals Inc.</applicant>.

XML files are read by a parser found in internet browsers. Style sheets provide the browser with the information to create tables, fonts, and colors for display.

The specific names of the element types and attributes as well as the valid syntax, structure and format for defining the XML elements are included in a file called document type declaration (DTD). If the XML document does not follow the DTD, then the file will not be able to be used properly.

The top three lines of the XML file should include the XML version, the style sheet type and address, and the DTD name and address.

Additional information about the XML standard can be found at the $\mathrm{W} 3 \mathrm{C}$ web site at http://www.w3c.org.

\section{SVG Files}

SVG is a language for describing two-dimensional graphics in XML. SVG allows for three types of graphic objects: vector graphic shapes (e.g., paths consisting of straight lines and curves), images and text. Graphical objects can be grouped, styled, transformed and composited into previously rendered objects. Text can be in any XML namespace suitable to the appplication, which enhances searchability and accessibility of the SVG graphics. The feature set includes nested transformations, clipping paths, alpha masks, filter effects, template objects and extensibility. 
SVG drawings can be dynamic and interactive. The Document Object Model (DOM) for SVG, which includes the full XML DOM, allows for straightforward and efficient vector graphics animation via scripting. A rich set of event handlers such as onmouseover and onclick can be assigned to any SVG graphical object. Because of its compatibility and leveraging of other Web standards, features like scripting can be done on SVG elements and other XML elements from different namespaces simultaneously within the same Web page. ${ }^{8}$

The specific use of SVG in a submission should be discussed with the regulatory authority.

\footnotetext{
${ }^{8}$ This description of SVG is from w3c web page http://www.w3c.org/graphics/svg
} 


\section{Appendix 8 XML eCTD DTD}

$<$ !-- eCTD Version 0.96 renamed to version 1.0

Jan 10 - Feb 6, 2002

Added keywords attribute to leaf element

This attribute is expected to be a comma-separated list of keywords

Removed 3+ level of detail in m2-4-nonclinical-overview

Removed 4+ level of detail in

m2-6-nonclinical-written-and-tabulated-summary

Removed 3+ level of detail in m2-7-clinical-summary

Removed 3+ level of detail in m2-5-clinical-overview

Changed name of leaf attribute md5-checksum to checksum

Added attribute checksum-type to leaf element

Added attribute manufacturer to

m2-3-p-drug-product and m3-2-p-drug-product

Removed 4+ level of detail in m2-3-quality-overall-summary

Removed 6+ level of detail in m3-2-p-2-pharmaceutical-development

$-->$

$<$ !-- eCTD Version 0.95 -->

$<!--$ Oct 20, 2001 -->

$<$ !-- Changes according to testing feedback -->

$<$ !-- See separate sheet -->

$<$ !-- eCTD Version $0.92 \quad-->$

$<$ !-- Changed incorrect m2-7-4-5-3 attribute -- $>$

$<$ !-- Added namespace info to leaf and xref elements -- $>$

$<$ !-- eCTD Version $0.91 \quad-->$

$<$ !- June 4, 2001 -->

$<$ !-- changed $\mathrm{m} 2-7-3$ to be 0 or more instead of 0 or 1 -->

$<$ !-- eCTD Version 0.9 -->

$<$ !-- ICH Tokyo Meeting: May 24, 2001 -->

$<$ !ENTITY \% att "ID ID \#IMPLIED

xml:lang CDATA \#IMPLIED " >

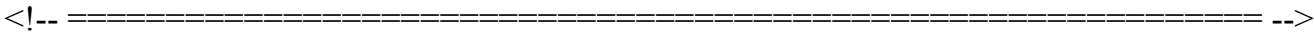

$<$ !-- Top-level element -->

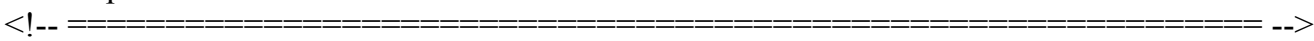

$<$ !ELEMENT ectd:ectd (m1-administrative-information-and-prescribing-information?, $\mathrm{m} 2$-common-technical-document-summaries?, m3-quality?, m4-nonclinical-study-reports?, m5-clinical-study-reports?

$<$ !ATTLIST ectd:ectd

$$
>
$$

xmlns:ectd CDATA \#FIXED "http://www.ich.org/ectd" xmlns:xlink CDATA \#FIXED "http://www.w3c.org/1999/xlink" xml:lang CDATA \#IMPLIED dtd-version CDATA \#FIXED "0.96" >

$<!--$

Page 8-1 


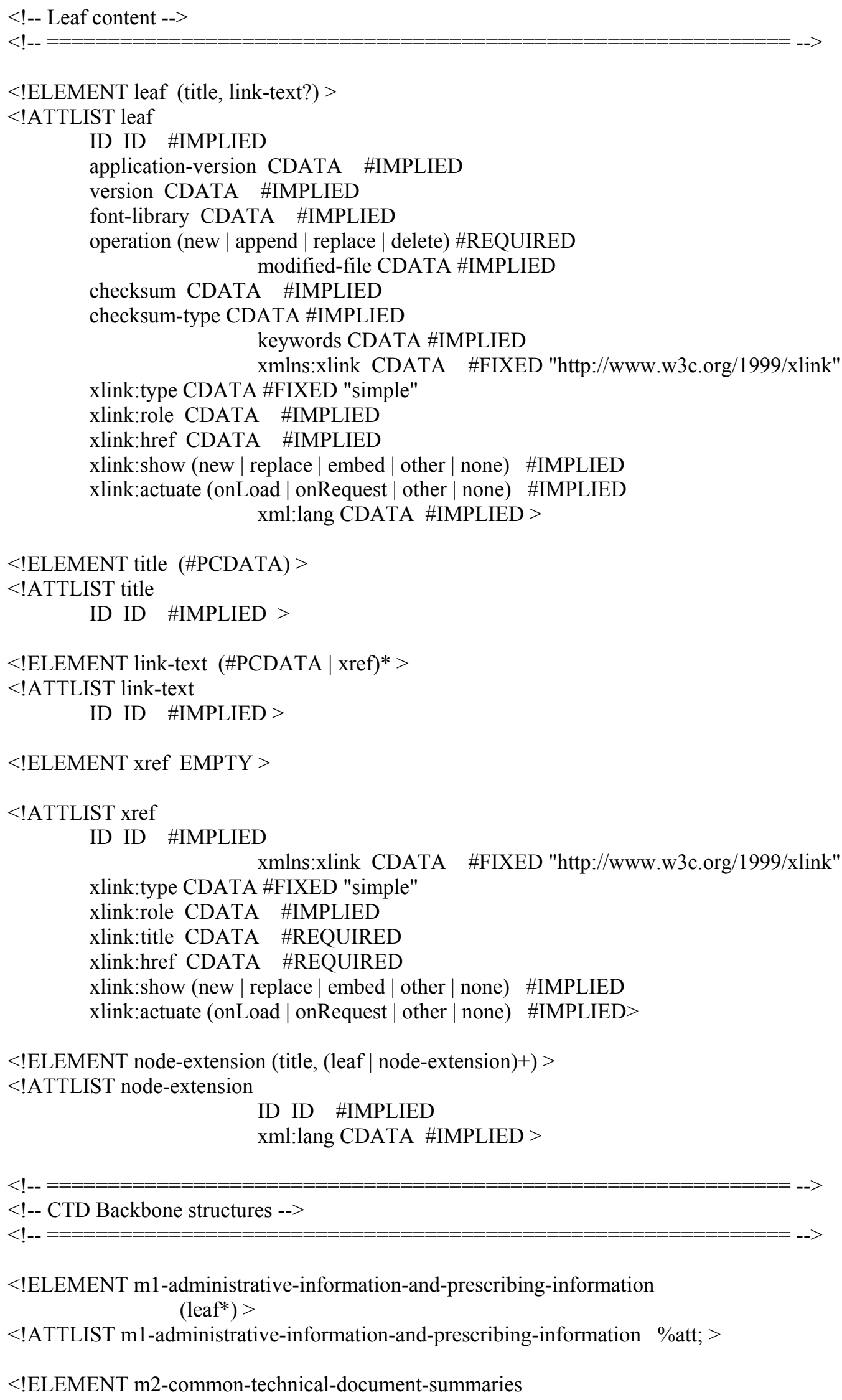


(leaf*,

m2-2-introduction?,

summary?,

$\mathrm{m} 2$-3-quality-overall-

overview?,

and-tabulated-summary?,

m2-4-nonclinical-

m2-5-clinical-overview?, m2-6-nonclinical-written-

$>$

m2-7-clinical-summary?)

$<$ !ATTLIST m2-common-technical-document-summaries \%att; >

$<$ !ELEMENT m2-2-introduction ((leaf $\mid$ node-extension) $\left.)^{*}\right)>$

$<$ !ATTLIST m2-2-introduction \%att; >

$<$ !ELEMENT m2-3-quality-overall-summary (leaf*,

introduction?,

substance*,

product*,

appendices?,

information?) $>$

$<$ !ATTLIST m2-3-quality-overall-summary \%att; >

$<$ !ELEMENT m2-3-introduction ((leaf $\mid$ node-extension)*) $>$

$<$ !ATTLIST m2-3-introduction \%att; >

$<$ !ELEMENT m2-3-s-drug-substance ((leaf | node-extension)*) $>$

$<$ !ATTLIST m2-3-s-drug-substance \%att;

substance CDATA \#REQUIRED

manufacturer CDATA \#REQUIRED >

$<$ !ELEMENT m2-3-p-drug-product ((leaf $\mid$ node-extension)*) $>$

$<$ !ATTLIST m2-3-p-drug-product \%att;

product-name CDATA \#IMPLIED

manufacturer CDATA \#IMPLIED >

dosageform CDATA \#IMPLIED

$<$ !ELEMENT m2-3-a-appendices ((leaf $\mid$ node-extension)*) $>$

$<$ !ATTLIST m2-3-a-appendices \%att; >

$<$ !ELEMENT m2-3-r-regional-information ((leaf $\mid$ node-extension $\left.)^{*}\right)>$

$<$ !ATTLIST m2-3-r-regional-information \%att; >

$<$ !ELEMENT m2-4-nonclinical-overview ((leaf $\mid$ node-extension)* $)^{*}>$

$<$ !ATTLIST m2-4-nonclinical-overview \%att; >

$<$ !ELEMENT m2-5-clinical-overview ((leaf $\mid$ node-extension)* $)^{*}>$

$<$ !ATTLIST m2-5-clinical-overview \%att; >

$<$ !ELEMENT m2-6-nonclinical-written-and-tabulated-summary (leaf*, 
summary?,

m2-6-1-introduction?, m2-6-2-pharmacology-written-summary?, $\mathrm{m} 2-6-3$-pharmacology-tabulated-summary?, m2-6-4-pharmacokinetics-written-

summary?,

m2-6-5-pharmacokinetics-tabulated-

$\mathrm{m} 2$-6-6-toxicology-written-summary?, $\mathrm{m} 2-6-7$-toxicology-tabulated-summary?) $>$

$<$ !ATTLIST m2-6-nonclinical-written-and-tabulated-summary \%att; >

$<$ !ELEMENT m2-6-1-introduction ((leaf $\mid$ node-extension)* $)^{*}>$

$<$ !ATTLIST m2-6-1-introduction \%att; >

$<$ !ELEMENT m2-6-2-pharmacology-written-summary ((leaf $\mid$ node-extension)*) $>$

$<$ !ATTLIST m2-6-2-pharmacology-written-summary \%att; >

$<$ !ELEMENT m2-6-3-pharmacology-tabulated-summary ((leaf $\mid$ node-extension)*) $>$

$<$ !ATTLIST m2-6-3-pharmacology-tabulated-summary \%att; >

$<$ !ELEMENT m2-6-4-pharmacokinetics-written-summary ((leaf $\mid$ node-extension)*) $>$

$<$ !ATTLIST m2-6-4-pharmacokinetics-written-summary \%att; >

$<$ !ELEMENT m2-6-5-pharmacokinetics-tabulated-summary ((leaf $\mid$ node-extension)*) $>$

$<$ !ATTLIST m2-6-5-pharmacokinetics-tabulated-summary \%att; >

$<$ !ELEMENT m2-6-6-toxicology-written-summary ((leaf $\mid$ node-extension)*) $>$

$<$ !ATTLIST m2-6-6-toxicology-written-summary \%att; >

$<$ !ELEMENT m2-6-7-toxicology-tabulated-summary ((leaf $\mid$ node-extension)* $)^{*}>$

$<$ !ATTLIST m2-6-7-toxicology-tabulated-summary \%att; >

$<$ !ELEMENT m2-7-clinical-summary (leaf*,

m2-7-1-summary-of-biopharmaceutic-and-associated-analytical-methods?,

m2-7-2-summary-of-clinical-pharmacology-studies?,

m2-7-3-summary-of-clinical-efficacy*,

m2-7-4-summary-of-clinical-safety?,

$\mathrm{m} 2-7-5$-references?,

m2-7-6-synopses-of-individual-studies?) >

$<$ !ATTLIST m2-7-clinical-summary \%att; >

$<$ !ELEMENT m2-7-1-summary-of-biopharmaceutic-and-associated-analytical-methods

((leaf $\mid$ node-extension $\left.)^{*}\right)>$

$<$ !ATTLIST m2-7-1-summary-of-biopharmaceutic-and-associated-analytical-methods \%att; >

$<$ !ELEMENT m2-7-2-summary-of-clinical-pharmacology-studies ((leaf $\mid$ node-extension)*) $>$

$<$ !ATTLIST m2-7-2-summary-of-clinical-pharmacology-studies \%att; >

$<$ !ELEMENT m2-7-3-summary-of-clinical-efficacy ((leaf $\mid$ node-extension)*) $>$

$<$ !ATTLIST m2-7-3-summary-of-clinical-efficacy \%att;

\#IMPLIED >

indication CDATA

$<$ !ELEMENT m2-7-4-summary-of-clinical-safety ((leaf $\mid$ node-extension)*) $>$

$<$ !ATTLIST m2-7-4-summary-of-clinical-safety \%att; > 
$<$ !ELEMENT m2-7-5-references ((leaf $\mid$ node-extension)*) $>$

$<$ !ATTLIST m2-7-5-references \%att; $>$

$<$ !ELEMENT m2-7-6-synopses-of-individual-studies ((leaf $\mid$ node-extension)*) $>$

$<$ !ATTLIST m2-7-6-synopses-of-individual-studies \%att; >

$<$ !ELEMENT m3-quality (leaf*,

$<$ !ATTLIST m3-quality \%att; >

m3-2-body-of-data?,

$\mathrm{m} 3$-3-literature-references?) $>$

$<$ !ELEMENT m3-2-body-of-data (leaf*,

m3-2-s-drug-substance*,

m3-2-p-drug-product*,

$\mathrm{m} 3$-2-a-appendices?,

$<$ !ATTLIST m3-2-body-of-data \%att; >

m3-2-r-regional-information?) >

$<$ !ELEMENT m3-2-s-drug-substance (leaf*,

m3-2-s-1-general-information?,

m3-2-s-2-manufacture?,

m3-2-s-3-characterisation?, m3-2-s-4-control-of-drug-substance?, m3-2-s-5-reference-standards-or-materials?, m3-2-s-6-container-closure-system?,

$<$ !ATTLIST m3-2-s-drug-substance \%att; m3-2-s-7-stability?) >

\#REQUIRED

\#REQUIRED >

$<$ !ELEMENT m3-2-s-1-general-information (leaf*,

m3-2-s-1-1-nomenclature?, m3-2-s-1-2-structure?,

$<$ !ATTLIST m3-2-s-1-general-information \%att; > m3-2-s-1-3-general-properties?) >

$<$ !ELEMENT m3-2-s-1-1-nomenclature ((leaf $\mid$ node-extension $\left.)^{*}\right)>$

$<$ !ATTLIST m3-2-s-1-1-nomenclature \%att; >

$<$ !ELEMENT m3-2-s-1-2-structure ((leaf $\mid$ node-extension) $\left.)^{*}\right)>$

$<$ !ATTLIST m3-2-s-1-2-structure \%att; >

$<$ !ELEMENT m3-2-s-1-3-general-properties ((leaf $\mid$ node-extension)*) $>$

$<$ !ATTLIST m3-2-s-1-3-general-properties \%att; >

$<$ !ELEMENT m3-2-s-2-manufacture (leaf*,

m3-2-s-2-1-manufacturer?,

controls?,

m3-2-s-2-2-description-of-manufacturing-process-and-process-

m3-2-s-2-3-control-of-materials?,

m3-2-s-2-4-controls-of-critical-steps-and-intermediates?,

$\mathrm{m} 3$-2-s-2-5-process-validation-and-or-evaluation?,

m3-2-s-2-6-manufacturing-process-development?) >

$<$ !ATTLIST m3-2-s-2-manufacture \%att; >

substance CDATA

manufacturer CDATA 
$<$ !ELEMENT m3-2-s-2-1-manufacturer ((leaf $\mid$ node-extension)* $)^{*}>$

$<$ !ATTLIST m3-2-s-2-1-manufacturer \%att; >

$<$ !ELEMENT m3-2-s-2-2-description-of-manufacturing-process-and-process-controls ((leaf $\mid$ nodeextension)* $)^{*}>$

$<$ !ATTLIST m3-2-s-2-2-description-of-manufacturing-process-and-process-controls \%att; >

$<$ !ELEMENT m3-2-s-2-3-control-of-materials ((leaf $\mid$ node-extension $\left.)^{*}\right)>$

$<$ !ATTLIST m3-2-s-2-3-control-of-materials \%att; >

$<$ !ELEMENT m3-2-s-2-4-controls-of-critical-steps-and-intermediates ((leaf $\mid$ node-extension)*) $>$

$<$ !ATTLIST m3-2-s-2-4-controls-of-critical-steps-and-intermediates \%att; >

$<$ !ELEMENT m3-2-s-2-5-process-validation-and-or-evaluation ((leaf $\mid$ node-extension)* ${ }^{*}>$

$<$ !ATTLIST m3-2-s-2-5-process-validation-and-or-evaluation \%att; >

$<$ !ELEMENT m3-2-s-2-6-manufacturing-process-development ((leaf $\mid$ node-extension)*) $>$

$<$ !ATTLIST m3-2-s-2-6-manufacturing-process-development \%att; >

$<$ !ELEMENT m3-2-s-3-characterisation (leaf*,

m3-2-s-3-1-elucidation-of-structure-and-other-characteristics?, m3-2-s-3-2-impurities?) >

$<$ !ATTLIST m3-2-s-3-characterisation \%att; >

$<$ !ELEMENT m3-2-s-3-1-elucidation-of-structure-and-other-characteristics

$\left((\text { leaf } \mid \text { node-extension })^{*}\right)>$

$<$ !ATTLIST m3-2-s-3-1-elucidation-of-structure-and-other-characteristics \%att; >

$<$ !ELEMENT m3-2-s-3-2-impurities ((leaf $\mid$ node-extension)*) $>$

$<$ !ATTLIST m3-2-s-3-2-impurities \%att; >

$<$ !ELEMENT m3-2-s-4-control-of-drug-substance (leaf*,

m3-2-s-4-1-specification?,

$\mathrm{m} 3-2-\mathrm{s}-4-2$-analytical-procedures?,

m3-2-s-4-3-validation-of-analytical-procedures?, m3-2-s-4-4-batch-analyses?,

$<$ !ATTLIST m3-2-s-4-control-of-drug-substance \%att; > m3-2-s-4-5-justification-of-specification?) >

$<$ !ELEMENT m3-2-s-4-1-specification $\left((\text { leaf } \mid \text { node-extension })^{*}\right)>$

$<$ !ATTLIST m3-2-s-4-1-specification \%att; >

$<$ !ELEMENT m3-2-s-4-2-analytical-procedures ((leaf $\mid$ node-extension)*) $>$

$<$ !ATTLIST m3-2-s-4-2-analytical-procedures \%att; >

$<$ !ELEMENT m3-2-s-4-3-validation-of-analytical-procedures ((leaf $\mid$ node-extension)* $)^{*}>$

$<$ !ATTLIST m3-2-s-4-3-validation-of-analytical-procedures \%att; >

$<$ !ELEMENT m3-2-s-4-4-batch-analyses ((leaf $\mid$ node-extension)*) $>$

$<$ !ATTLIST m3-2-s-4-4-batch-analyses \%att; >

$<$ !ELEMENT m3-2-s-4-5-justification-of-specification ((leaf $\mid$ node-extension)*) $>$

$<$ !ATTLIST m3-2-s-4-5-justification-of-specification \%att; >

$<$ !ELEMENT m3-2-s-5-reference-standards-or-materials ((leaf $\mid$ node-extension)*) $>$ 
$<$ !ATTLIST m3-2-s-5-reference-standards-or-materials \%att; >

$<$ !ELEMENT m3-2-s-6-container-closure-system ((leaf $\mid$ node-extension)*) $>$

$<$ !ATTLIST m3-2-s-6-container-closure-system \%att; >

$<$ !ELEMENT m3-2-s-7-stability (leaf*, m3-2-s-7-1-stability-summary-and-conclusions?, m3-2-s-7-2-post-approval-stability-protocol-and-stability-commitment?, m3-2-s-7-3-stability-data?) >

$<$ !ATTLIST m3-2-s-7-stability \%att; >

$<$ !ELEMENT m3-2-s-7-1-stability-summary-and-conclusions ((leaf $\mid$ node-extension)*) $>$

$<$ !ATTLIST m3-2-s-7-1-stability-summary-and-conclusions \%att; >

$<$ !ELEMENT m3-2-s-7-2-post-approval-stability-protocol-and-stability-commitment

$\left((\text { leaf } \mid \text { node-extension })^{*}\right)>$

$<$ !ATTLIST m3-2-s-7-2-post-approval-stability-protocol-and-stability-commitment \%att; >

$<$ !ELEMENT m3-2-s-7-3-stability-data ((leaf $\mid$ node-extension)* $)^{*}>$

$<$ !ATTLIST m3-2-s-7-3-stability-data \%att; >

$<$ !ELEMENT m3-2-p-drug-product (leaf*,

m3-2-p-1-description-and-composition-of-the-drug-product?, m3-2-p-2-pharmaceutical-development?, m3-2-p-3-manufacture?,

m3-2-p-4-control-of-excipients*, $\mathrm{m} 3-2-\mathrm{p}-5$-control-of-drug-product?, m3-2-p-6-reference-standards-or-materials?, m3-2-p-7-container-closure-system?, m3-2-p-8-stability?) >

$<$ !ATTLIST m3-2-p-drug-product \%att;

\#IMPLIED

\#IMPLIED

product-name CDATA

dosageform CDATA

$>$

$<$ !ELEMENT m3-2-p-1-description-and-composition-of-the-drug-product

((leaf $\mid$ node-extension $\left.)^{*}\right)$

$<$ !ATTLIST m3-2-p-1-description-and-composition-of-the-drug-product \%att; >

$<$ !ELEMENT m3-2-p-2-pharmaceutical-development (leaf*,

m3-2-p-2-1-components-of-the-drug-product?, $\mathrm{m} 3-2-\mathrm{p}-2-2$-drug-product?, m3-2-p-2-3-manufacturing-process-development?, m3-2-p-2-4-container-closure-system?, m3-2-p-2-5-microbiological-attributes?,

$<$ !ATTLIST m3-2-p-2-pharmaceutical-development \%att; > m3-2-p-2-6-compatibility?) >

$<$ !ELEMENT m3-2-p-2-1-components-of-the-drug-product $\left((\text { leaf } \mid \text { node-extension })^{*}\right)>$

$<$ !ATTLIST m3-2-p-2-1-components-of-the-drug-product \%att; >

$<$ !ELEMENT m3-2-p-2-2-drug-product ((leaf $\mid$ node-extension)*) $>$

$<$ !ATTLIST m3-2-p-2-2-drug-product \%att; >

Page 8-7 
$<$ !ELEMENT m3-2-p-2-3-manufacturing-process-development ((leaf $\mid$ node-extension $\left.)^{*}\right)>$

$<$ !ATTLIST m3-2-p-2-3-manufacturing-process-development \%att; >

$<$ !ELEMENT m3-2-p-2-4-container-closure-system ((leaf $\mid$ node-extension)* $)^{*}>$

$<$ !ATTLIST m3-2-p-2-4-container-closure-system \%att; >

$<$ !ELEMENT m3-2-p-2-5-microbiological-attributes ((leaf $\mid$ node-extension)* $)^{*}>$

$<$ !ATTLIST m3-2-p-2-5-microbiological-attributes \%att; >

$<$ !ELEMENT m3-2-p-2-6-compatibility ((leaf $\mid$ node-extension)* $)>$

$<$ !ATTLIST m3-2-p-2-6-compatibility \%att; >

$<$ !ELEMENT m3-2-p-3-manufacture (leaf*,

m3-2-p-3-1-manufacturers?,

m3-2-p-3-2-batch-formula?,

m3-2-p-3-3-description-of-manufacturing-process-and-process-controls?,

m3-2-p-3-4-controls-of-critical-steps-and-intermediates?,

m3-2-p-3-5-process-validation-and-or-evaluation?) $>$

$<$ !ATTLIST m3-2-p-3-manufacture \%att; >

$<$ !ELEMENT m3-2-p-3-1-manufacturers ((leaf $\mid$ node-extension)*) $>$

$<$ !ATTLIST m3-2-p-3-1-manufacturers \%att; >

$<$ !ELEMENT m3-2-p-3-2-batch-formula ((leaf $\mid$ node-extension)*) $>$

$<$ !ATTLIST m3-2-p-3-2-batch-formula \%att; >

$<$ !ELEMENT m3-2-p-3-3-description-of-manufacturing-process-and-process-controls

node-extension)*) $>$

$(($ leaf $\mid$

$<$ !ATTLIST m3-2-p-3-3-description-of-manufacturing-process-and-process-controls \%att; >

$<$ !ELEMENT m3-2-p-3-4-controls-of-critical-steps-and-intermediates

node-extension)*) $>$

((leaf $\mid$

$<$ !ATTLIST m3-2-p-3-4-controls-of-critical-steps-and-intermediates \%att; >

$<$ !ELEMENT m3-2-p-3-5-process-validation-and-or-evaluation $\left((\text { leaf } \mid \text { node-extension })^{*}\right)>$

$<$ !ATTLIST m3-2-p-3-5-process-validation-and-or-evaluation \%att; >

$<$ !ELEMENT m3-2-p-4-control-of-excipients (leaf*,

m3-2-p-4-1-specifications?,

$\mathrm{m} 3-2-\mathrm{p}-4-2$-analytical-procedures?,

m3-2-p-4-3-validation-of-analytical-procedures?,

m3-2-p-4-4-justification-of-specifications?,

m3-2-p-4-5-excipients-of-human-or-animal-origin?,

m3-2-p-4-6-novel-excipients?) >

$<$ !ATTLIST m3-2-p-4-control-of-excipients \%att;

excipient CDATA \#IMPLIED >

$<$ !ELEMENT m3-2-p-4-1-specifications ((leaf | node-extension)*) $>$

$<$ !ATTLIST m3-2-p-4-1-specifications \%att; >

$<$ !ELEMENT m3-2-p-4-2-analytical-procedures ((leaf $\mid$ node-extension)* $)^{*}>$

$<$ !ATTLIST m3-2-p-4-2-analytical-procedures \%att; > 
$<$ !ELEMENT m3-2-p-4-3-validation-of-analytical-procedures ((leaf $\mid$ node-extension $\left.)^{*}\right)>$

$<$ !ATTLIST m3-2-p-4-3-validation-of-analytical-procedures \%att; >

$<$ !ELEMENT m3-2-p-4-4-justification-of-specifications ((leaf $\mid$ node-extension $\left.)^{*}\right)>$

$<$ !ATTLIST m3-2-p-4-4-justification-of-specifications \%att; >

$<$ !ELEMENT m3-2-p-4-5-excipients-of-human-or-animal-origin ((leaf $\mid$ node-extension $\left.)^{*}\right)>$

$<$ !ATTLIST m3-2-p-4-5-excipients-of-human-or-animal-origin \%att; >

$<$ !ELEMENT m3-2-p-4-6-novel-excipients ((leaf $\mid$ node-extension)* $)>$

$<$ !ATTLIST m3-2-p-4-6-novel-excipients \%att; >

$<$ !ELEMENT m3-2-p-5-control-of-drug-product (leaf*,

m3-2-p-5-1-specifications?,

$\mathrm{m} 3-2-\mathrm{p}-5-2$-analytical-procedures?,

m3-2-p-5-3-validation-of-analytical-procedures?, m3-2-p-5-4-batch-analyses?,

$\mathrm{m} 3-2-\mathrm{p}-5-5$-characterisation-of-impurities?,

$<$ !ATTLIST m3-2-p-5-control-of-drug-product \%att; > m3-2-p-5-6-justification-of-specifications?) >

$<$ !ELEMENT m3-2-p-5-1-specifications ((leaf $\mid$ node-extension)* $)>$

$<$ !ATTLIST m3-2-p-5-1-specifications \%att; >

$<$ !ELEMENT m3-2-p-5-2-analytical-procedures ((leaf $\mid$ node-extension $\left.)^{*}\right)>$

$<$ !ATTLIST m3-2-p-5-2-analytical-procedures \%att; >

$<$ !ELEMENT m3-2-p-5-3-validation-of-analytical-procedures ((leaf $\mid$ node-extension)*) $>$

$<$ !ATTLIST m3-2-p-5-3-validation-of-analytical-procedures \%att; >

$<$ !ELEMENT m3-2-p-5-4-batch-analyses ((leaf $\mid$ node-extension)*) $>$

$<$ !ATTLIST m3-2-p-5-4-batch-analyses \%att; >

$<$ !ELEMENT m3-2-p-5-5-characterisation-of-impurities ((leaf $\mid$ node-extension)*) $>$

$<$ !ATTLIST m3-2-p-5-5-characterisation-of-impurities \%att; >

$<$ !ELEMENT m3-2-p-5-6-justification-of-specifications ((leaf $\mid$ node-extension $\left.)^{*}\right)>$

$<$ !ATTLIST m3-2-p-5-6-justification-of-specifications \%att; >

$<$ !ELEMENT m3-2-p-6-reference-standards-or-materials ((leaf $\mid$ node-extension $\left.)^{*}\right)>$

$<$ !ATTLIST m3-2-p-6-reference-standards-or-materials \%att; >

$<$ !ELEMENT m3-2-p-7-container-closure-system ((leaf $\mid$ node-extension)*) $>$

$<$ !ATTLIST m3-2-p-7-container-closure-system \%att; >

$<$ !ELEMENT m3-2-p-8-stability (leaf*,

m3-2-p-8-1-stability-summary-and-conclusion?,

m3-2-p-8-2-post-approval-stability-protocol-and-stability-commitment?,

$<$ !ATTLIST m3-2-p-8-stability \%att; >

m3-2-p-8-3-stability-data?) $>$

$<$ !ELEMENT m3-2-p-8-1-stability-summary-and-conclusion ((leaf $\mid$ node-extension)*) $>$

$<$ !ATTLIST m3-2-p-8-1-stability-summary-and-conclusion \%att; >

$<$ !ELEMENT m3-2-p-8-2-post-approval-stability-protocol-and-stability-commitment 
extension $\left.)^{*}\right)>$

((leaf $\mid$ node-

$<$ !ATTLIST m3-2-p-8-2-post-approval-stability-protocol-and-stability-commitment \%att; >

$<$ !ELEMENT m3-2-p-8-3-stability-data ((leaf $\mid$ node-extension $\left.)^{*}\right)>$

$<$ !ATTLIST m3-2-p-8-3-stability-data \%att; >

$<$ !ELEMENT m3-2-a-appendices (leaf*,

m3-2-a-1-facilities-and-equipment?,

$\mathrm{m} 3-2-\mathrm{a}-2$-adventitious-agents-safety-evaluation?,

$<$ !ATTLIST m3-2-a-appendices \%att; >

m3-2-a-3-novel-excipients?) >

$<$ !ELEMENT m3-2-a-1-facilities-and-equipment ((leaf $\mid$ node-extension $\left.)^{*}\right)>$

$<$ !ATTLIST m3-2-a-1-facilities-and-equipment \%att; >

$<$ !ELEMENT m3-2-a-2-adventitious-agents-safety-evaluation ((leaf $\mid$ node-extension)*) $>$

$<$ !ATTLIST m3-2-a-2-adventitious-agents-safety-evaluation \%att; >

$<$ !ELEMENT m3-2-a-3-novel-excipients ((leaf $\mid$ node-extension)*) $>$

$<$ !ATTLIST m3-2-a-3-novel-excipients \%att; >

$<$ !ELEMENT m3-2-r-regional-information ((leaf $\mid$ node-extension)*) $>$

$<$ !ATTLIST m3-2-r-regional-information \%att; >

$<$ !ELEMENT m3-3-literature-references ((leaf $\mid$ node-extension)* $)^{*}>$

$<$ !ATTLIST m3-3-literature-references \%att; >

$<$ !ELEMENT m4-nonclinical-study-reports (leaf*,

m4-2-study-reports?, m4-3-literature-references?) >

$<$ !ATTLIST m4-nonclinical-study-reports \%att; >

$<$ !ELEMENT m4-2-study-reports (leaf*,

m4-2-1-pharmacology?, m4-2-2-pharmacokinetics?, $\mathrm{m} 4-2-3$-toxicology?, m4-2-4-local-tolerance?, m4-2-5-other-toxicity-studies?) >

$<$ !ATTLIST m4-2-study-reports \%att; >

$<$ !ELEMENT m4-2-1-pharmacology (leaf*,

m4-2-1-1-primary-pharmacodynamics?, m4-2-1-2-secondary-pharmacodynamics?, m4-2-1-3-safety-pharmacology?,

$<$ !ATTLIST m4-2-1-pharmacology \%att; >

m4-2-1-4-pharmacodynamic-drug-interactions?) >

$<$ !ELEMENT m4-2-1-1-primary-pharmacodynamics ((leaf $\mid$ node-extension)*) $>$

$<$ !ATTLIST m4-2-1-1-primary-pharmacodynamics \%att; >

$<$ !ELEMENT m4-2-1-2-secondary-pharmacodynamics ((leaf $\mid$ node-extension)* $)>$

$<$ !ATTLIST m4-2-1-2-secondary-pharmacodynamics \%att; >

$<$ !ELEMENT m4-2-1-3-safety-pharmacology $\left((\text { leaf } \mid \text { node-extension })^{*}\right)>$

$<$ !ATTLIST m4-2-1-3-safety-pharmacology \%att; > 
$<$ !ELEMENT m4-2-1-4-pharmacodynamic-drug-interactions ((leaf $\mid$ node-extension)*) $>$

$<$ !ATTLIST m4-2-1-4-pharmacodynamic-drug-interactions \%att; >

$<$ !ELEMENT m4-2-2-pharmacokinetics (leaf*,

m4-2-2-1-analytical-methods-and-validation-reports?, m4-2-2-2-absorption?, m4-2-2-3-distribution?, m4-2-2-4-metabolism?, $\mathrm{m} 4-2-2-5$-excretion?, m4-2-2-6-pharmacokinetic-drug-interactions?,

$<$ !ATTLIST m4-2-2-pharmacokinetics \%att; > m4-2-2-7-other-pharmacokinetic-studies?) >

$<$ !ELEMENT m4-2-2-1-analytical-methods-and-validation-reports $>$ ((leaf $\mid$ node-extension $\left.)^{*}\right)$

$<$ !ATTLIST m4-2-2-1-analytical-methods-and-validation-reports \%att; >

$<$ !ELEMENT m4-2-2-2-absorption ((leaf $\mid$ node-extension $\left.)^{*}\right)>$ $<$ !ATTLIST m4-2-2-2-absorption \%att; >

$<$ !ELEMENT m4-2-2-3-distribution ((leaf $\mid$ node-extension)*) $>$

$<$ !ATTLIST m4-2-2-3-distribution \%att; >

$<$ !ELEMENT m4-2-2-4-metabolism ((leaf $\mid$ node-extension)*) $>$

$<$ !ATTLIST m4-2-2-4-metabolism \%att; >

$<$ !ELEMENT m4-2-2-5-excretion ((leaf $\mid$ node-extension $\left.)^{*}\right)>$

$<$ !ATTLIST m4-2-2-5-excretion \%att; >

$<$ !ELEMENT m4-2-2-6-pharmacokinetic-drug-interactions ((leaf $\mid$ node-extension)* $)^{*}>$

$<$ !ATTLIST m4-2-2-6-pharmacokinetic-drug-interactions \%att; >

$<$ !ELEMENT m4-2-2-7-other-pharmacokinetic-studies ((leaf $\mid$ node-extension)*) $>$

$<$ !ATTLIST m4-2-2-7-other-pharmacokinetic-studies \%att; >

$<$ !ELEMENT m4-2-3-toxicology (leaf*,

m4-2-3-1-single-dose-toxicity?,

m4-2-3-2-repeat-dose-toxicity?,

m4-2-3-3-genotoxicity?,

$\mathrm{m} 4-2-3-4-c a r c i n o g e n i c i t y ?$

$<$ !ATTLIST m4-2-3-toxicology \%att; >

m4-2-3-5-reproductive-and-developmental-toxicity?) $>$

$<$ !ELEMENT m4-2-3-1-single-dose-toxicity $\left((\text { leaf } \mid \text { node-extension })^{*}\right)>$

$<$ !ATTLIST m4-2-3-1-single-dose-toxicity \%att; >

$<$ !ELEMENT m4-2-3-2-repeat-dose-toxicity $(($ leaf $\mid$ node-extension)* $)>$

$<$ !ATTLIST m4-2-3-2-repeat-dose-toxicity \%att; >

$<$ !ELEMENT m4-2-3-3-genotoxicity (leaf*,

m4-2-3-3-1-in-vitro?

$<$ !ATTLIST m4-2-3-3-genotoxicity \%att; >

m4-2-3-3-2-in-vivo?) >

Page 8-11 
$<$ !ELEMENT m4-2-3-3-1-in-vitro ((leaf $\mid$ node-extension)* $)^{*}>$

$<$ !ATTLIST m4-2-3-3-1-in-vitro \%att; >

$<$ !ELEMENT m4-2-3-3-2-in-vivo ((leaf $\mid$ node-extension $\left.)^{*}\right)>$

$<$ !ATTLIST m4-2-3-3-2-in-vivo \%att; >

$<$ !ELEMENT m4-2-3-4-carcinogenicity (leaf*, m4-2-3-4-1-long-term-studies?, m4-2-3-4-2-short-or-medium-term-studies?, m4-2-3-4-3-other-studies?) $>$

$<$ !ATTLIST m4-2-3-4-carcinogenicity \%att; >

$<$ !ELEMENT m4-2-3-4-1-long-term-studies ((leaf $\mid$ node-extension)* $)>$

$<$ !ATTLIST m4-2-3-4-1-long-term-studies \%att; >

$<$ !ELEMENT m4-2-3-4-2-short-or-medium-term-studies ((leaf $\mid$ node-extension)*) $>$

$<$ !ATTLIST m4-2-3-4-2-short-or-medium-term-studies \%att; >

$<$ !ELEMENT m4-2-3-4-3-other-studies ((leaf $\mid$ node-extension)* $)>$

$<$ !ATTLIST m4-2-3-4-3-other-studies \%att; >

$<$ !ELEMENT m4-2-3-5-reproductive-and-developmental-toxicity (leaf*, m4-2-3-5-1-fertility-and-early-embryonic-development?, m4-2-3-5-2-embryo-fetal-development?, m4-2-3-5-3-prenatal-and-postnatal-development-including-maternal-function?,

evaluated?) $>$ m4-2-3-5-4-studies-in-which-the-offspring-juvenile-animals-are-dosed-and-or-further-

$<$ !ATTLIST m4-2-3-5-reproductive-and-developmental-toxicity \%att; >

$<$ !ELEMENT m4-2-3-5-1-fertility-and-early-embryonic-development ((leaf $\mid$ node-extension)*) $>$

$<$ !ATTLIST m4-2-3-5-1-fertility-and-early-embryonic-development \%att; >

$<$ !ELEMENT m4-2-3-5-2-embryo-fetal-development ((leaf $\mid$ node-extension)*) $>$

$<$ !ATTLIST m4-2-3-5-2-embryo-fetal-development \%att; >

$<$ !ELEMENT m4-2-3-5-3-prenatal-and-postnatal-development-including-maternal-function $\left((\text { leaf } \mid \text { node-extension })^{*}\right)>$

$<$ !ATTLIST m4-2-3-5-3-prenatal-and-postnatal-development-including-maternal-function $\%$ att; >

$<$ !ELEMENT m4-2-3-5-4-studies-in-which-the-offspring-juvenile-animals-are-dosed-and-or-furtherevaluated ((leaf $\mid$ node-extension)*) $>$

$<$ !ATTLIST m4-2-3-5-4-studies-in-which-the-offspring-juvenile-animals-are-dosed-and-or-furtherevaluated \%att; >

$<$ !ELEMENT m4-2-4-local-tolerance ((leaf $\mid$ node-extension)*) $>$

$<$ !ATTLIST m4-2-4-local-tolerance \%att; >

$<$ !ELEMENT m4-2-5-other-toxicity-studies (leaf*,

m4-2-5-1-antigenicity?,

m4-2-5-2-immunotoxicity?,

m4-2-5-3-mechanistic-studies?,

m4-2-5-4-dependence?,

m4-2-5-5-metabolites?,

m4-2-5-6-impurities?,

m4-2-5-7-other?) >

Page 8-12 
$<$ !ATTLIST m4-2-5-other-toxicity-studies \%att; >

$<$ !ELEMENT m4-2-5-1-antigenicity ((leaf $\mid$ node-extension $\left.)^{*}\right)>$

$<$ !ATTLIST m4-2-5-1-antigenicity \%att; >

$<$ !ELEMENT m4-2-5-2-immunotoxicity ((leaf $\mid$ node-extension)*) $>$

$<$ !ATTLIST m4-2-5-2-immunotoxicity \%att; >

$<$ !ELEMENT m4-2-5-3-mechanistic-studies ((leaf $\mid$ node-extension $\left.)^{*}\right)>$

$<$ !ATTLIST m4-2-5-3-mechanistic-studies \%att; >

$<$ !ELEMENT m4-2-5-4-dependence ((leaf $\mid$ node-extension)*) $>$

$<$ !ATTLIST m4-2-5-4-dependence \%att; >

$<$ !ELEMENT m4-2-5-5-metabolites ((leaf $\mid$ node-extension)*) $>$

$<$ !ATTLIST m4-2-5-5-metabolites \%att; >

$<$ !ELEMENT m4-2-5-6-impurities ((leaf $\mid$ node-extension)*) $>$

$<$ !ATTLIST m4-2-5-6-impurities \%att; >

$<$ !ELEMENT m4-2-5-7-other ((leaf $\mid$ node-extension $\left.)^{*}\right)>$

$<$ !ATTLIST m4-2-5-7-other \%att; >

$<$ !ELEMENT m4-3-literature-references ((leaf $\mid$ node-extension)* $)>$

$<$ !ATTLIST m4-3-literature-references \%att; >

$<$ !ELEMENT m5-clinical-study-reports (leaf*,

$<$ !ATTLIST m5-clinical-study-reports \%att; >

m5-2-tabular-listing-of-all-clinical-studies?, m5-3-clinical-study-reports?, m5-4-literature-references?) >

$<$ !ELEMENT m5-2-tabular-listing-of-all-clinical-studies ((leaf | node-extension)*)>

$<$ !ATTLIST m5-2-tabular-listing-of-all-clinical-studies \%att; >

$<$ !ELEMENT m5-3-clinical-study-reports (leaf*,

m5-3-1-reports-of-biopharmaceutic-studies?,

m5-3-2-reports-of-studies-pertinent-to-pharmacokinetics-

using-human-biomaterials?,

m5-3-3-reports-of-human-pharmacokinetics-pk-studies?, m5-3-4-reports-of-human-pharmacodynamics-pd-studies?, m5-3-5-reports-of-efficacy-and-safety-studies*, m5-3-6-reports-of-postmarketing-experience?, m5-3-7-case-report-forms-and-individual-patient-listings?) $>$

$<$ !ATTLIST m5-3-clinical-study-reports \%att; >

$<$ !ELEMENT m5-3-1-reports-of-biopharmaceutic-studies (leaf*, m5-3-1-1-bioavailability-study-reports?, m5-3-1-2-comparative-ba-and-bioequivalence-study-reports?, m5-3-1-3-in-vitro-in-vivo-correlation-study-reports?, m5-3-1-4-reports-of-bioanalytical-and-analytical-methods-for-human-studies?) >

$<$ !ATTLIST m5-3-1-reports-of-biopharmaceutic-studies \%att; >

$<$ !ELEMENT m5-3-1-1-bioavailability-study-reports ((leaf $\mid$ node-extension)*) $>$

$<$ !ATTLIST m5-3-1-1-bioavailability-study-reports \%att; > 
$<$ !ELEMENT m5-3-1-2-comparative-ba-and-bioequivalence-study-reports

$\left((\text { leaf } \mid \text { node-extension })^{*}\right)>$

$<$ !ATTLIST m5-3-1-2-comparative-ba-and-bioequivalence-study-reports \%att; >

$<$ !ELEMENT m5-3-1-3-in-vitro-in-vivo-correlation-study-reports ((leaf $\mid$ node-extension)*) $>$

$<$ !ATTLIST m5-3-1-3-in-vitro-in-vivo-correlation-study-reports \%att; >

$<$ !ELEMENT m5-3-1-4-reports-of-bioanalytical-and-analytical-methods-for-human-studies

extension)* $)^{*}>$

((leaf $\mid$ node-

$<$ !ATTLIST m5-3-1-4-reports-of-bioanalytical-and-analytical-methods-for-human-studies

$\%$ att; >

$<$ !ELEMENT m5-3-2-reports-of-studies-pertinent-to-pharmacokinetics-using-human-biomaterials

(leaf*,

m5-3-2-1-plasma-protein-binding-study-reports?,

m5-3-2-2-reports-of-hepatic-metabolism-and-drug-interaction-studies?,

m5-3-2-3-reports-of-studies-using-other-human-biomaterials?) >

$<$ !ATTLIST m5-3-2-reports-of-studies-pertinent-to-pharmacokinetics-using-human-biomaterials \%att; >

$<$ !ELEMENT m5-3-2-1-plasma-protein-binding-study-reports ((leaf $\mid$ node-extension)* $)>$

$<$ !ATTLIST m5-3-2-1-plasma-protein-binding-study-reports \%att; >

$<$ !ELEMENT m5-3-2-2-reports-of-hepatic-metabolism-and-drug-interaction-studies

$\left((\text { leaf } \mid \text { node-extension })^{*}\right)>$

$<$ !ATTLIST m5-3-2-2-reports-of-hepatic-metabolism-and-drug-interaction-studies \%att; >

$<$ !ELEMENT m5-3-2-3-reports-of-studies-using-other-human-biomaterials

$\left((\text { leaf } \mid \text { node-extension })^{*}\right)>$

$<$ !ATTLIST m5-3-2-3-reports-of-studies-using-other-human-biomaterials \%att; >

$<$ !ELEMENT m5-3-3-reports-of-human-pharmacokinetics-pk-studies

(leaf*,

m5-3-3-1-healthy-subject-pk-and-initial-tolerability-study-reports?,

m5-3-3-2-patient-pk-and-initial-tolerability-study-reports?,

m5-3-3-3-intrinsic-factor-pk-study-reports?,

m5-3-3-4-extrinsic-factor-pk-study-reports?,

m5-3-3-5-population-pk-study-reports?) $>$

$<$ !ATTLIST m5-3-3-reports-of-human-pharmacokinetics-pk-studies \%att; >

$<$ !ELEMENT m5-3-3-1-healthy-subject-pk-and-initial-tolerability-study-reports

$\left((\text { leaf } \mid \text { node-extension })^{*}\right)>$

$<$ !ATTLIST m5-3-3-1-healthy-subject-pk-and-initial-tolerability-study-reports \%att; >

$<$ !ELEMENT m5-3-3-2-patient-pk-and-initial-tolerability-study-reports

$<$ !ATTLIST m5-3-3-2-patient-pk-and-initial-tolerability-study-reports \%att; >

$\left((\text { leaf } \mid \text { node-extension })^{*}\right)>$

$<$ !ELEMENT m5-3-3-3-intrinsic-factor-pk-study-reports ((leaf $\mid$ node-extension $\left.)^{*}\right)>$

$<$ !ATTLIST m5-3-3-3-intrinsic-factor-pk-study-reports \%att; >

$<$ !ELEMENT m5-3-3-4-extrinsic-factor-pk-study-reports ((leaf $\mid$ node-extension)*) $>$

$<$ !ATTLIST m5-3-3-4-extrinsic-factor-pk-study-reports \%att; >

$<$ !ELEMENT m5-3-3-5-population-pk-study-reports ((leaf $\mid$ node-extension)*) $>$

$<$ !ATTLIST m5-3-3-5-population-pk-study-reports \%att; > 
$<$ !ELEMENT m5-3-4-reports-of-human-pharmacodynamics-pd-studies

(leaf*,

m5-3-4-1-healthy-subject-pd-and-pk-pd-study-reports?, m5-3-4-2-patient-pd-and-pk-pd-study-reports?) >

$<$ !ATTLIST m5-3-4-reports-of-human-pharmacodynamics-pd-studies \%att; >

$<$ !ELEMENT m5-3-4-1-healthy-subject-pd-and-pk-pd-study-reports

$\left((\text { leaf } \mid \text { node-extension })^{*}\right)>$

$<$ !ATTLIST m5-3-4-1-healthy-subject-pd-and-pk-pd-study-reports \%att; >

$<$ !ELEMENT m5-3-4-2-patient-pd-and-pk-pd-study-reports ((leaf $\mid$ node-extension)*) $>$

$<$ !ATTLIST m5-3-4-2-patient-pd-and-pk-pd-study-reports \%att; >

$<$ !ELEMENT m5-3-5-reports-of-efficacy-and-safety-studies (leaf*, m5-3-5-1-study-reports-of-controlled-clinical-studies-

pertinent-to-the-claimed-indication?,

m5-3-5-2-study-reports-of-uncontrolled-clinical-studies?,

study?, m5-3-5-3-reports-of-analyses-of-data-from-more-than-one-

$<$ !ATTLIST m5-3-5-reports-of-efficacy-and-safety-studies \%att; indication CDATA \#IMPLIED >

$<$ !ELEMENT m5-3-5-1-study-reports-of-controlled-clinical-studies-pertinent-to-the-claimed-indication $\left((\text { leaf } \mid \text { node-extension })^{*}\right)>$

$<$ !ATTLIST m5-3-5-1-study-reports-of-controlled-clinical-studies-pertinent-to-the-claimed-indication

\%att; >

$<$ !ELEMENT m5-3-5-2-study-reports-of-uncontrolled-clinical-studies ((leaf $\mid$ node-extension)*) $>$

$<$ !ATTLIST m5-3-5-2-study-reports-of-uncontrolled-clinical-studies \%att; >

$<$ !ELEMENT m5-3-5-3-reports-of-analyses-of-data-from-more-than-one-study

$\left((\text { leaf } \mid \text { node-extension })^{*}\right)>$

$<$ !ATTLIST m5-3-5-3-reports-of-analyses-of-data-from-more-than-one-study \%att; >

$<$ !ELEMENT m5-3-5-4-other-study-reports ((leaf | node-extension)*) $>$

$<$ !ATTLIST m5-3-5-4-other-study-reports \%att; >

$<$ !ELEMENT m5-3-6-reports-of-postmarketing-experience ((leaf $\mid$ node-extension)*) $>$

$<$ !ATTLIST m5-3-6-reports-of-postmarketing-experience \%att; >

$<$ !ELEMENT m5-3-7-case-report-forms-and-individual-patient-listings

$$
\left((\text { leaf } \mid \text { node-extension })^{*}\right)>
$$

$<$ !ATTLIST m5-3-7-case-report-forms-and-individual-patient-listings \%att; >

$<$ !ELEMENT m5-4-literature-references ((leaf $\mid$ node-extension)* $)^{*}>$

$<$ !ATTLIST m5-4-literature-references \%att; > 


\section{Appendix 9 Glossary}

The intended content of this section is the definition of terms used in the set of documentation associated with the eCTD.

\section{Architecture}

A general term for the design and construction of computer systems, including technical infrastructure, information (data), and applications.

\section{ASCII}

American Standard Code for Information Interchange. A specification for representing text as computer-readable information.

\section{Browser}

A program which allows the user to read hypertext, to view contents of web pages, and to navigate from one page to another, e.g., Netscape Navigator, Mosaic, Microsoft Internet Explorer.

\section{Common Technical Document (CTD)}

A harmonized format for a regulatory dossier that is considered acceptable in Japan, Europe, United States and Canada.

\section{Decryption}

To reverse encryption.

\section{Directory (see also Folder)}

The operating system method of organizing and providing access to individual files. Also called a Folder.

\section{DTD}

Document Type Definition. A hierarchical organization or representation of the information contents of a document utilized by SGML or XML.

eCTD

The electronic format of the ICH Common Technical Document

\section{Encryption}

The process of reversibly confusing text or data using a secret formula.

\section{EWG}

Expert Working Group.

Folder (see also Directory) 
The operating system method of organizing and providing access to individual files. Also called a Directory.

\section{HTML}

Hypertext Markup Language. Commonly used to format Web pages.

\section{Hypertext}

A system that enables links to be established between specific words or figures in a document to other text, tables or image allowing quick access to the linked items (such as on the World Wide Web).

\section{ICH}

International Conference on Harmonisation of Technical Requirements for Registration of Pharmaceuticals for Human Use.

\section{Infrastructure}

The basic support services for computing; the hardware, operating system, and network on which applications and data are stored and on which the database management systems run.

\section{Internet}

The world-wide network of computers for accessing, sending, sharing, and transferring information between sites at different locations. It is uncontrolled and unadministered, and when you connect to the Internet, you actually become a part of it.

\section{ISO}

International Standards Organization - founded in 1946, it is the principal international standards-setting organization.

\section{Leaf}

The eCTD DTD XML element that describes the content to be provided. The leaf consists of a file and the meta-data associated with that file. Such files are placed in a directory structure that is similar to branches of a tree.

\section{Logical Document}

One or more CTD table of contents sections that together contain the minimum amount of information to be exchanged. Ideally, this is a single physical file.

\section{M2}

Multidisciplinary Group 2 (ESTRI) of ICH.

\section{Network}

A communication system which connects different computers and enables them to share peripherals such as printers, disk drives and databases. Users (clients) can access applications and databases connected by the network.

\section{Node Extension}


The extension of the definition of an element beneath a defined table of contents tag.

\section{PDF}

Portable Document Format - a proprietary (Adobe Systems) de-facto standard for the electronic transfer of documents.

\section{SGML}

Standardized Generalized Markup Language. An ISO standard for describing structured information in a platform independent manner.

\section{Software or Software Application}

Computer programs or applications. There are two principle types: system software, e.g., computer operating system or a utility program (sometimes called a driver) for printing and application software, e.g., an accounts package or CAD program.

\section{Standard}

A technical specification that addresses a business requirement, has been implemented in viable commercial products, and, to the extent practical, complies with recognized standards organizations such as ISO.

\section{Web page}

Any page on the World Wide Web. The page usually offers the reader the ability to jump to other topics of interest.

\section{World Wide Web (WWW)}

Segment of the Internet offering point and click (hypertext) access to information, as text, image or sound, on an enormous number of topics from around the world.

\section{XML}

Extensible Markup Language. An ISO standard for describing structured information in a platform-independent manner. 